MEASUREMENTS AND CALCULATIOUS

3. OF WATER VELOCIT, MOMENTUM MUY, ANO RELATED FLOW PARAMETERS OBNAINED FAOM SINGLEPHASW WATER WTECRAL ACCEPTANCE TESTS OF THE PKL TNSTMUMENTED SPOOL PTECES

tretsetseti:

Otthet +197

\title{
MASTER
}

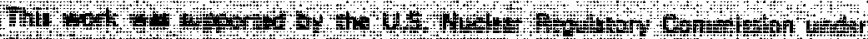

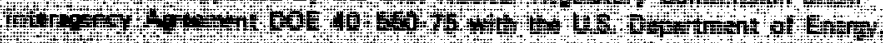

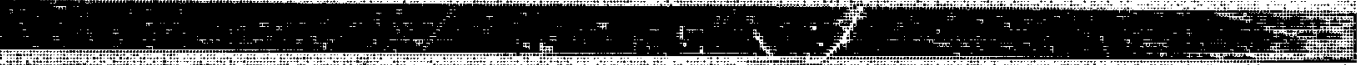

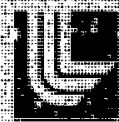

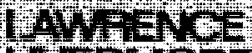

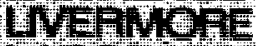
Wention
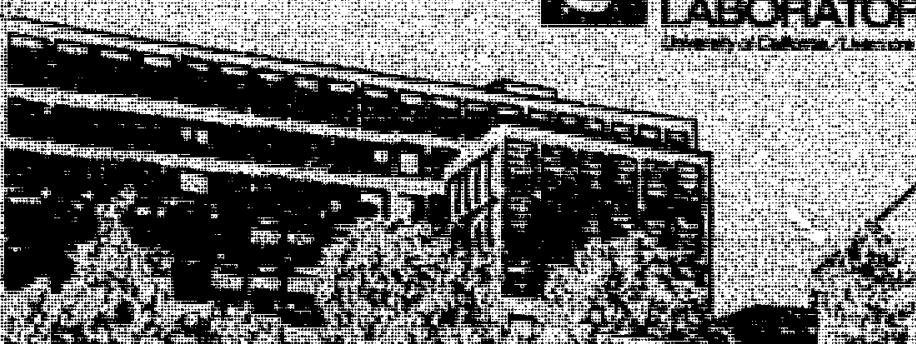


\title{
近
}

LAWRENCE LIVERMORE IABORATORY

University of Cahtornia Livermore,California 94550

\section{MEASUREMENTS AND CALCULATIONS OF WATER VELOCITY, MOMENTUM FLUX, AND RELATED FLOW PARAMETERS OBTAINED FROM SINGLE-PHASE WATER INTEGRAL ACCEPTANCE TESTS OF THE PKL INSTRUMENTED SPOOL PIECES}

\author{
Werner Stein
}

Manuscript date: October 9, 1979

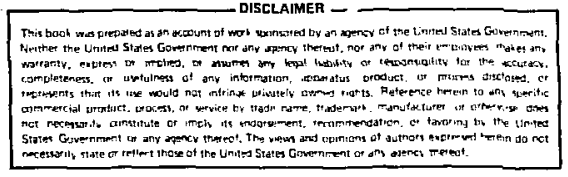


CONTENTS

Abstract

Introduction

PKL Spool Piece Design and Instrumentation.

Spool Piece Physical Description

Spool Piece Instrumentation

Turbine Flowmeter

Drag Screen System

Absolute and Differential Pressure Measurements = = . . . 4

Wall, Fluid, and Steam Temperature Measurements . . . . . 4

Densitometer System . . . . . . . . . . . . 4

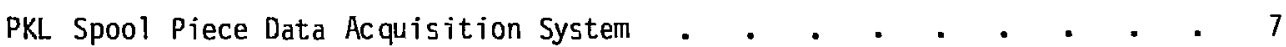

System Description . • • • • • • • • • . . 7

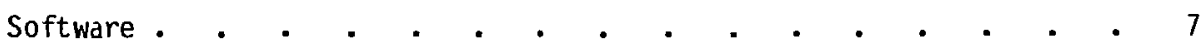

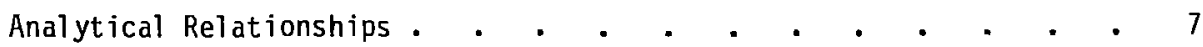

Engineering Unit Conversions. • . • • • • • . . 8

Flow Parameter Calculations . • . • • • . . . . . . 9

PKL Water-Flow Test Facility . • . • • • . . . . . 15

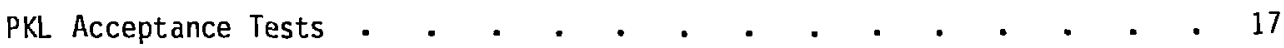

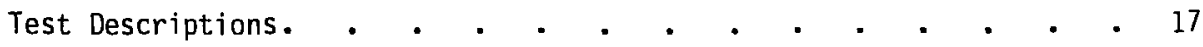

Orifice-Meter Results. . . . . . . . . . . . . . 19

Spool Test Results • • • • • • . • • • • • . . 20

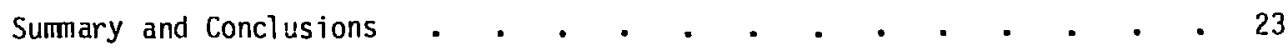

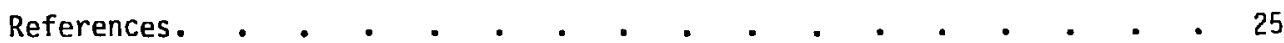

Appendix A PKL Spool Piece Drawings (EG\&G Drawing Nos. 137086, 137087, and 137088) . . • . . . • . 27

Appendix B PKL Spool Piece Test Results Data. . . . . . . . . 37 


\section{LIST OF ILLUSTRATION}

1. PKL water-flow test facility schematic . . . . . . . . 16

\section{LIST OF TABLES}

1. Densitometer statistical error analysis . . . . . . . . 6

2. PKL test facility orifice-meter test results . . . . . . . 18

3. PKL spool piece water-flow test results . • • • • • • 21 


\section{ABSTRACT}

The operation of the emergency core cooling system and its related steam-binding problems in pressurized water reactors is the subject of a cooperative study by the United States, Germany, and Japan. Lawrence Livermore Laboratory and EG\&G, Inc., San Ramon Operations, are responsible for the design, hardware, and software of the $80.8-\mathrm{mm}$ and 113-imm spool piece measurement systems for the German Primarkreislauf (PKL) Test Facility at Kraftwerk Union in Erlangen, West Germany. This work was done for the U. S. Nuclear Regulatory Comission, Division of Reactor Safety Research, under its 3-D Technical Support and Instrumentation Program.

Four instrumented spools capable of measuring individual phase parameters in two-phase flows were constructed. Each spoot contains a flow turbine, drag screen, three-beain densitometer, and pressure and temperature probes. A computerized data acquisition system is also provided to store and analyze data from the four spools.

The four spools were shipped to the PKL Test Facility in West Germany for acceptance testing in a water-flow loop. Spool measuraments of velocity and momentum flux were compared to the values obtained from an orifice meter installed in the loop piping system.

The turbine flowmeter velocity data for all tests were within allowable tolerances. Drag screen momentum flux measurements were also within tolerance with the exception of a few points. 
INTRODUCTION

The operation of the emergency core cooling system (ECCS) and its related steambinding probleins in pressurized water reactors (PWR's) is the subject of a cooperative German, Japanese, and United States steam-binding study. The German Primarkreislauf (PKL) Test Facility at Kraftwerk Inion (KWU) in Erlangen, West Germany, was constructed to perform loss-of-coolant experiment (LOCE) reflood tests. In order to measure fluid flows during these reflood tests, a spool piece measurement system* was developed to measure both single-phase and twophase water and stean flows in the various coolant loop legs of the PKL reactor. Lawrence Livermore Laboratory (LLL) and EG\&G, Inc., San Ramon Operations, developed the spool piece measurement system for the U. S. Nuclear Regulatory Comission, Division of Reactor Safety Research (NRC-RSR) under its 3-D Technical Support and Instrumentation Program.

The spool piece measurement systen basically consists of four spools and a computerized data acquisition system (DAS). Each spool piece contains a flow turbine, drag screen, three-beari densitometer, and pressure and temperature probes. The DAS is capable of receiving and storing data signals, converting data to engineering unit values, and calculating flow parameters in two-phase flow.

The contractual testing requirements for the spools call for prototype testing of the spools in single-phase water flow and in two-phase water-stearn flows [1] and acceptance testing in single-phase water at the PKL Test Facility. The acceptance testing is the subject of this report.

The acceptance testing involved disassembling the spool pieces and related electronic equipment for packing and shipping to the PKL Test Facility in West Gerinany. The spools and related equipment were reassembled at the PKL Test Facility and checked for proper operation.

\footnotetext{
*In this report, the spool piece measurement system is also referred to as a spool piece or a spool.
} 
Testing involved placing the individual pipe spools in the PKL. water-flow loop and then flowing water through the spool at low, nominal, and high flow rates. Comparisons of spool-determined velocities and monentum fluxes were made with velocities and monentum fluxes obtained from an orifice-meter instrunentation system installed on the PKL water-flow loop. 
PKL SPOOL PIECE DESIGN AND INSTRUMENTATION

SPOOL PIECE PHYSICAL DESCRIPTION

A total of four spool piece measurenent systems were tested at the PKL Test Facility. Each spool consists of a flanged pipe that is $830 \mathrm{~mm}$ long. Three of the spools have an inside diameter of $80.8 \mathrm{~mm}$, and one spool has an inside diameter of $113 \mathrm{~mm}$. The spools are designed for horizontal installation except for one of the $80.8-\mathrm{mm}$ spools which is designed for vertical installation.

Each spool contains the following instruments:

- Three-beam densitometer.

- Full-dianeter flow turbine.

- Full-dianeter drag screen or plate.

- Wall temperature probe.

- Fluid temperature probe.

- Superheated-stean temperature probe (vertical spool only).

- Absolute pressure probe.

- Differential pressure probe across the drag screen.

The pertinent physical details for each of the spools are given in EG\&G drawing nos. 13700 f, 137087 , and 137088 (refer to Appendix A).

SPOOL PIECE INSTRUMENTATION

A description of the instrumentation used on the spool pieces is given below. For each system, accuracy of $1 \%$ of full scale is required, and a response time of $350 \mathrm{~ms}$ is required for a 10 to $90 \%$ response due to a step excitation.

\section{Turbine Flowmeter}

A whole-duct, bidirectional turbine flowmeter is used to measure volumetric flow in the snool picce. Passage of fluid through the instrument causes the ninebladed "stor to turn at a rate proportional to the flow rate of the fluid. The 
rotation of the rotor is sensed by two magnetic pickup devices, and the direction of rotation is determined by the phase shift between these two devices. The flowmeter consists of the rotor, stainless-steel ball bearings, and flow straighteners attached to both ends of a stationary shaft. The range of operation is from 2 to $60 \mathrm{~m} / \mathrm{s}$ in steam-flow conditions.

\section{Drag Screen System}

A full pipe drag screen or plate is used to measure momentum flux in the spool piece. The drag screen is held in place by a three-pin suspension system. Screen deflections, under flow conditions, are sensed by three variable reluctance transducers. The sum of the three outputs is proportional to the momentum flux. The range of operation is from 70 to $7,200 \mathrm{~kg} / \mathrm{m}-5^{2}$.

\section{Absolute and Differential Pressure Measurements}

The fluid differential pressure is measured across the drag screen, and the absolute pressure is measured upstream of the drag screen. Both measurements are made using water-cooled, water-filled standoff tubes connecting the transducers to the spool piece. The absolute pressure transducer has a range of operation from 0 to $700 \mathrm{kPa}$. The differential pressure transducer has a range of operation from 0 to $7 \mathrm{kPa}$.

Wall, Fluid, and Steam Temperature Measurements

Probes consisting of chromel-aluinel thermocouples and an ice-bath reference are used to measure the wall, fluid, and superheated-steam temperatures in the spool piece.

\section{Densitometer System}

The density of single-phase and two-phase flow is measured by a three-beam, low-energy $(6-22 \mathrm{keV}) \mathrm{X}$-ray densitometer. The chordal average density is determined by the amount of attenuation of the radiation beain by the fluid in the spool. The densitoneter contains a single silicon-lithium-drifted, low-energy 
photon detector collimated th three sources through the beryllium window of the spool piece. The radiation sources are Iron-55, Cadmium-109, and Americiun-241. The range of measurement is from 0.7 to $1,000 \mathrm{~kg} / \mathrm{m}^{3}$. A densitometer statistical error analysis ${ }^{[2]}$ is given in Table 1. 


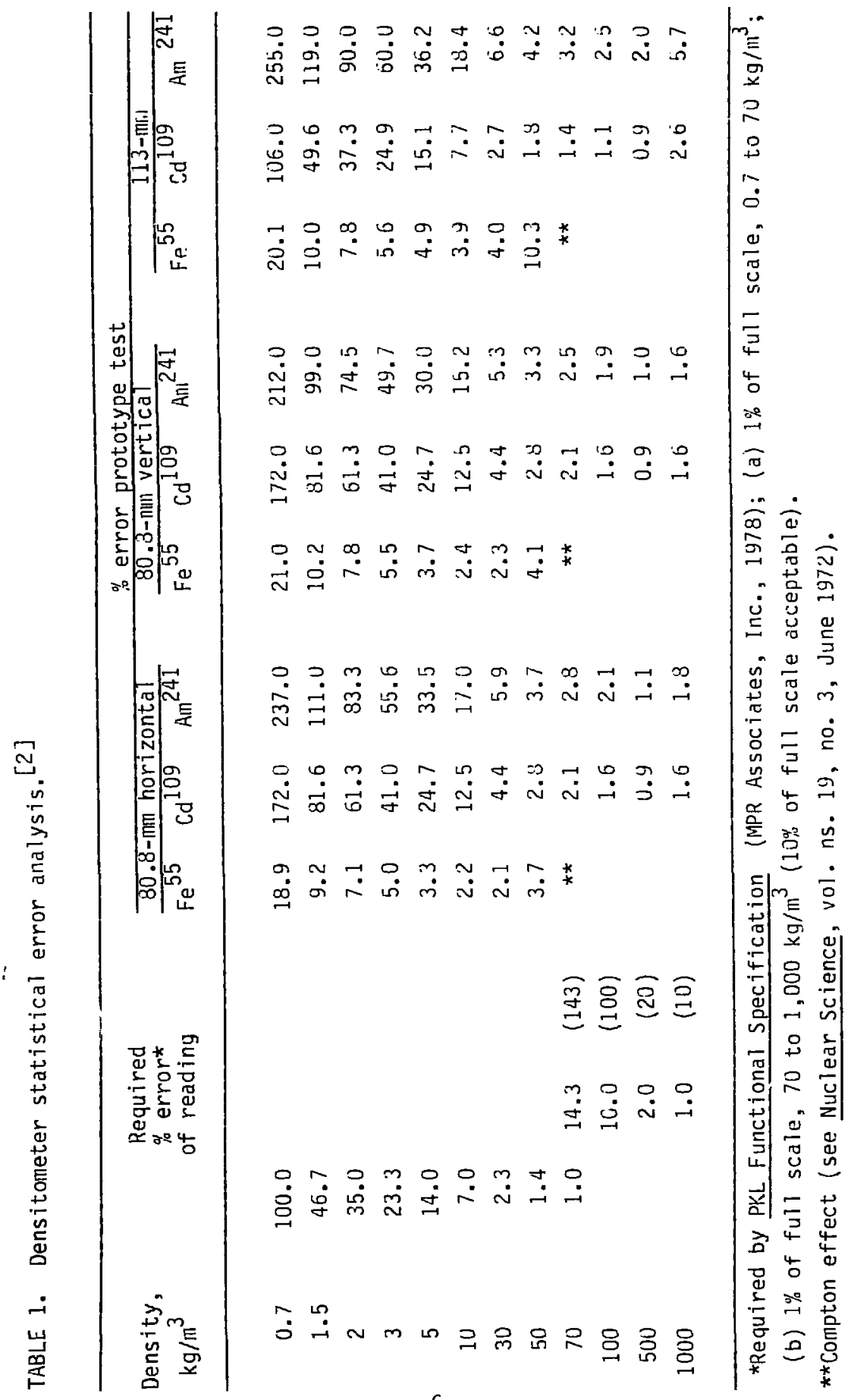




\section{PKL SPOOL PIECE DATA ACUUISITION SYSTEM}

SYSTEM DESCRIPTION

The computer system used to monitor and control spool piece testing is an LSI-11 Iilicroprocessor systen with 32,000 words of ilemory. The peripherals include the following:

- Line printer.

- CRT teminal (TTY).

- Cartridge tape recorder/rejroducer.

- Analog-to-digital converter and multiplexer.

- Three tioppy-disc drives.

SOFTWARE

Coliputer prograns were developed to allow the acquisition of 50 data channels and to provide the capabilities for:

- Data arquisition and storage on cartridge tape.

- Data retrieval froin tape and analysis of the data.

- Real-tine acquisition and amalysis of the data.

The analysis of the data consists of converting the data signats to engineering units. This is accomplished primarily by a calibration-table, look-up scheine with straight-line interpolation between the points. After conversion of the data to engineering units, calculations are rade to determine the two-phase flow parameters.

\section{ANALYTICAL RELATIONSHIPS}

A major portion of the calculations involves converting the data signals into engineering units, and then using these engineering unit values to deternine the phase velocities, mass flow rate, and voif fraction. 
Data signals, received from the following channels, are first converted ro voltage valuts, which are then used to deternine the appropriate engineering unit values from arailable calibration tables:

- Wall temperature $\left({ }^{\circ} \mathrm{C}\right)$.

- Fluid temperature $\left({ }^{0} \mathrm{C}\right)$.

- Steam temperature $\left({ }^{0} \mathrm{C}\right)$.

- Fluid absulute pressure (kPa).

- Differential pressure (kPa).

- Flow turbine velocit.y $(\mathrm{m} / \mathrm{s})$.

- Individual arag screen transducer force (iv).

Ta determine velocity froin the flow turbine, two calibration tables are asailable. One table is based on a water calibration and the other on an air alibration.

To : fetermine momentuin flux from the trag screen, calibration tables relatiny total furce to moinentin flux are available. The total force is deterinined by algebraically sumiling the forca values frolil the threa trag transducers. If one of the drag transducers is over-range, an alternate calculation for total forci is outained from the differential pressure transducer signal.

The density along each of the three bedin paths is obtained from the voltage sigilal. The voltaye signal is given by the following relationship: ${ }^{3}$

$$
V=V_{o f f}+D e^{-C \rho}
$$

1;here

$$
\begin{aligned}
& V=\text { voltage signal } \\
& V_{\text {off }}=\text { offset voltage in system } \\
& D \quad=\text { calibration constait proportional to the radiation source intensity }
\end{aligned}
$$




$$
\begin{aligned}
& C=\text { attenuation calihration constant } \\
& p \quad=\text { averduge density along bean path. }
\end{aligned}
$$

Engineering unit values for density along each bean are obtained by inverting equation (I) to qive:

$$
\mu=\frac{1}{C} \ln \frac{D}{\sqrt{-V_{\text {Dff }}}}
$$

\section{Flow Parameter Calculitions}

The individual phase paraneters calculated include tensity, velocity, mass flow rate, void fraction, and average pipe cross-sectional density.

Density. The vapor density $\left(\rho_{S}\right)$ and ? iquid density $\left(p_{y,}\right)$ are calculated fro:u stean table routines with neasured $v_{3}$ ues of fluid temperature and pressure as input. The average cross-sectional censity is calculated by fitting a density distribution model to the individual bean density measurements. [4]

Individual Phase Velocities ${ }^{[5]}$ and Void Fraction. The average density of two-phase flow is given by:

$$
\rho_{f}=x \rho_{S}+(1-x) \rho_{s}
$$

where

$$
\begin{aligned}
& \alpha=\text { void fraction } \\
& \rho_{S}=\text { steam density } \\
& \rho_{\Delta, /}=\text { water density. }
\end{aligned}
$$


The void fraction is given by solving equation (3):

$$
x=\frac{P_{W}-P_{f}}{H_{W}-i_{S}}
$$

where

$$
a_{f}=\text { average density. }
$$

The mass flux $\left(i_{f}\right)$ is given by:

$$
G_{f}=\rho_{f} V_{f}=u p_{S} V_{S}+(1-\alpha) \rho_{W} V_{W}
$$

where

$$
V_{f}=\text { average velocity. }
$$

Solving equation (4) for $v_{f}$ gives:

$$
V_{f}=\frac{G_{f}}{\rho_{f}}=\frac{\alpha \rho_{S} V_{S}+(1-\alpha) \rho_{W} V_{W}}{\alpha \rho_{S}+\left(1-\alpha ! \rho_{W}\right.}
$$

The rnomentum $f l u x$ (I) is measured from the drag screen and is given by:

$$
I=\alpha p_{S} v_{S}^{2}+(1-\alpha) p_{W}^{2} v_{W}
$$

The turbine velocity $\left(v_{t}\right)$ is calculated, based on the Rouhani model, ${ }^{[6]}$ as:

$$
V_{t}=\frac{I}{\rho_{f} V_{f}}=\frac{\alpha \rho_{S} V_{S}^{2}+(1-\alpha) \rho_{W}^{2} V_{W}}{\alpha \rho_{S} V_{S}+(1-\alpha) \rho_{W} V_{W}} \text {. }
$$

The drag screen and turbine outputs above have been normalized by their cal ibration constants. 
Another form of this gquation sinows that the Kouhani :lodel is a flowing quality weighted velocity ds follows:

$$
v_{t}=x v_{S}+(1-x) v_{W}
$$

where

$$
x=\text { flowing qudity. }
$$

Solving equations (5) and (7) sianltaneously gives che following individual phase velocities:

$$
\begin{aligned}
& v_{S}=V_{f}+n^{-1 / 2} \Delta V \\
& v_{b d}=V_{f}-n^{1 / 2} \Delta V
\end{aligned}
$$

where

$$
\begin{aligned}
& n=\left(\frac{u}{1-\alpha}\right)\left(\frac{\rho_{S}}{\rho_{i N}}\right) \\
& \Delta V=\sqrt{I_{f} V_{t}-V_{f}^{L}} \\
& V_{f}=\frac{I}{\rho_{f} V_{t}} .
\end{aligned}
$$

From an analysis of equation (7), the turbine valocity $\left(V_{t}\right)$ is equal to or greater than the average fluid velucity $\left(V_{f}\right)$. Therefore, fron equation (13), the following is obtained:

$$
\frac{I}{v_{t}^{2}} \leq \rho_{f}
$$

and, in general, the following relationship holds true:

$$
\rho_{S} \leq \frac{I}{v_{t}^{2}} \leq p_{f} \leq p_{W} .
$$


The relationship given by equation (15) is one of the tests used in the software to deterinine if the data is satisfactory for proceeding with the calculations. If the test is not found to hold true, then unity slip nodels are assumed. If the average density $\left(\rho_{F}\right)$ is found not. to satisfy the following test

$$
\rho_{S} \leq \rho_{f} \leq \rho_{W} \quad
$$

then the unity slip velocity (VUS) is assualed to aqual the curbine velocity $\left(V_{t}\right)$ and the density $\left(\rho_{f}\right)$ is set equal to $I / V_{t}^{2}$. If the following test,

$$
\rho_{S} \leq \frac{1}{v_{t}^{2}} \leq \rho_{f}
$$

is not satisfied, the VUS is calculated from:

$$
\text { VUS }=\sqrt{\frac{1}{\rho_{f}}} .
$$

Mass flow Rate. The calculations for ilass flow rates for single-phase and two-phase water and stearn flow conditions are presented below.

Single-Phase Water Flow Conditions. The nass flow rato (MU) for single-phase water flow conditions is calculated by the following relationship:

$$
M A=A \times P_{W} \times V_{W}
$$

where

$$
\begin{aligned}
& \Lambda=\text { pipe cross-sectional area } \\
& V_{W}=\text { water velority. }
\end{aligned}
$$


$V_{W}$ is calculated from the relationship:

$$
V_{W}=\sqrt{\frac{1}{\rho_{i d}}} \text {. }
$$

Single-Phase Stedin Flow Conditions. For single-phuse stean flow conditions, the inass flow rate (MS) is calculated by the following relationship:

$$
M S=\Lambda \times \rho_{S} \times V_{S}
$$

where

$$
v_{S}=\text { turbine velocity reading based on an air calibration. }
$$

Two-Phase flow Conditions. For the case of two-phase flow, the stean inass flow rates (MS) and the water inass flow rates (MW) are given by:

$$
\text { MS }=A \times a \times \rho_{S} \times V_{S}
$$

and

$$
M W=A \times(1-\varepsilon) \times P_{W} \times V_{W}
$$

where $V_{S}$ and $V_{W}$ are obtained from equations $(9)$ and $(10)$, respectively. 
The PKL water-flow test facility was designed for testing the four sponls in single-phase water flows. The spools were flange-inounted in the facility piping. The facility allowed flow in one direction only, but the flow direction in the horizontal spools could be reversed by turning the spools around and installing thein in reverse order. The vertical spool cannot be turned upside down and, therefore, testing was possible only in one flow direction. A schematic of the water-flow test facility is shown in Fig. 1.

Operation of the facility during testing involvad starting the punp and manually opening the control valva to obtain an orifice-neter signal that corrasponded to i desired flow rate. 


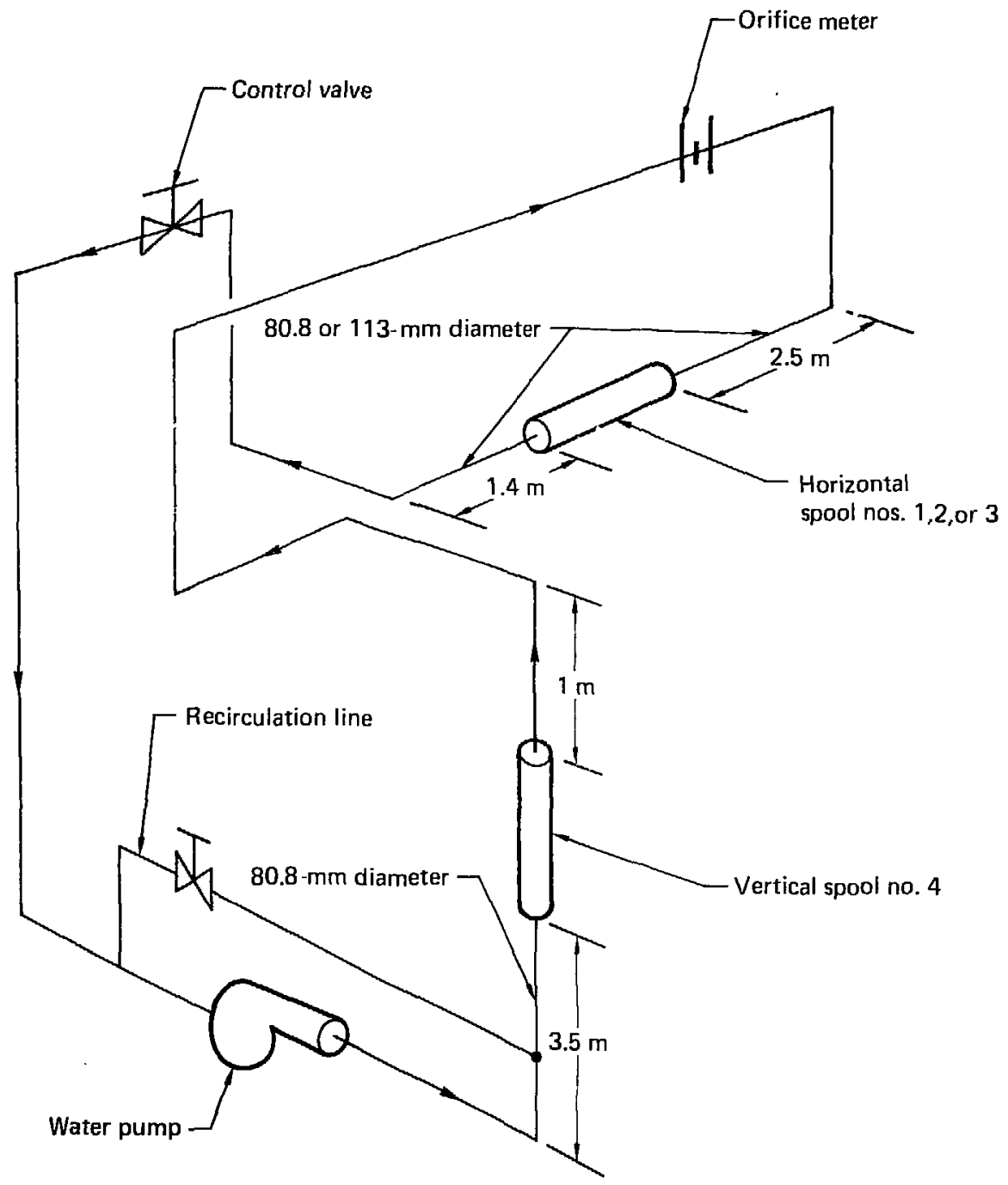

Figure 1. PKL water-flow test facility schematic. 


\section{TEST DESCRIPTIONS}

The four spools tested are identified below:

- Spool no. 1-.-80.8-mm diameter, right-hand, horizontal spool.

- Spool no. 2--80.8-mm diameter, left-hand, horizontal spool.

- Spool no. 3--113-mm diameter, horizontal spool.

- Spool no. 4--80.8-rm diameter, vertical spool.

Testing was intended to be in accordance with the acceptance testing plan, [7] with each spool undergoing water-flow rates representative of the following momentum fluxes:

\begin{tabular}{lr} 
Water-flow rate & Momentum flux \\
\hline Low flow condition & 72 \\
Nominal flow condition & 700 \\
High flow condition & 7,200
\end{tabular}

Due to a malfunction of the control valve in the PKL flow loop, the high-flow condition was not attainable for tests inve'ving spool nos. 2 and 3 . Spool nos. 1 and 2 were tested in both forward flow and reverse flow conditions. Spool no. 3 was tested only in forward flow due to a complete failure of the loop control valve in reverse flow tests. Spool no. 4 was tested only in reverse flow.

The testing procedure involved initially establishing a low flow rate through the spool. The spool UAS was started as soon as the initial flow rate appeared steady. After maintaining this flow rate for one minute the flow rate was increased to the nominal f?ow condition and then to the high flow condition with flow maintained For one minute at eacn condition. From the high flow condition, the flow was decreased to the nominal flow condition and then back down to the low flow sondition. The time to complete this test sequence was on the order of $500 \mathrm{~s}$, with the DAS collecting data every $10 \mathrm{~s}$ for this entire sequence. 
Each test sequence was given a test number startinij with number 5 . The computer printout of engineering unit data and calculated flow parameters for all test sequencas is given in Appendix 3 . Within each test sequence, five steady flow conditions representative of low, noilinal, and high flow rates are usually established and they are each identified by a letter (i.e., A, B, C, D, or E) conbined with the test sequence number (e.g., 5A). The tine interval vitinin each sequence which is applicable to a particular test is jiven in Table 2 in the "Time Interval" coldinn.

TABLE 2. PKL test facility orifice-meter test results.

\begin{tabular}{|c|c|c|c|c|c|c|c|c|}
\hline \multirow{2}{*}{$\begin{array}{l}\text { Test } \\
\text { no. }\end{array}$} & \multirow{2}{*}{$\begin{array}{c}\text { Time } \\
\text { interval, } \\
\text { s }\end{array}$} & \multicolumn{4}{|c|}{ Urifice-meter signal, mA } & \multirow{2}{*}{$\begin{array}{l}\text { Densiby, } \\
\mathrm{kg} / \mathrm{in}\end{array}$} & \multirow{2}{*}{$\begin{array}{c}\text { Calculated } \\
\text { velocity, } \\
\text { m/s }\end{array}$} & \multirow{2}{*}{$\begin{array}{c}\text { Calculated } \\
\text { moment } \\
\mathrm{kg} / \mathrm{m}-\mathrm{s}^{2}\end{array}$} \\
\hline & & $\mathrm{High}$ & Low & Average & Offset & & & \\
\hline $5 A$ & $0-50$ & 8.13 & 3.11 & 3.12 & 7.99 & 994 & 0.303 & 34 \\
\hline 58 & $130-194$ & 3.97 & 3.95 & 3.90 & 7.99 & 394 & 0.341 & 702 \\
\hline $5 C$ & $330-390$ & 13.10 & 17.60 & 17.35 & 7.99 & 393 & 2.631 & 7,138 \\
\hline 51 & $495-555$ & 9.00 & 3.97 & $\therefore 335$ & 7.99 & 993 & 0.852 & 724 \\
\hline $5 \mathrm{E}$ & $592-631)$ & 3.10 & $3.0^{\prime} y$ & 3.095 & 7.99 & 993 & 0.277 & 76 \\
\hline $5 F$ & $720-770$ & 10.10 & 17.93 & 13.015 & 7.99 & 993 & 2.703 & 7,257 \\
\hline $6 \lambda$ & $0-6 !$ & 8.10 & 8.09 & 3.095 & 3.0 & $9 y 4$ & 0.263 & 59 \\
\hline 63 & $130-180$ & 8.97 & 8.96 & 8.965 & 8.0 & 993 & 0.839 & 599 \\
\hline $6 C$ & $275-330$ & 18.02 & 17.63 & 17.35 & 3.0 & 993 & 2.680 & 7,131 \\
\hline 63 & $430-475$ & 8.97 & 8.96 & 3.965 & 3.0 & 993 & 0.339 & 699 \\
\hline $5 E$ & $490-520$ & 8.10 & 8.09 & 3.095 & 8.0 & 993 & 0.263 & 69 \\
\hline $7 A$ & $9-60$ & 8.11 & 3.10 & 3.115 & 8.0 & 993 & 0.277 & 76 \\
\hline $7 \mathrm{~B}$ & $120-130$ & 3.98 & 3.95 & 3.97 & 3.0 & 993 & 0.341 & 702 \\
\hline $7 C$ & $240-300$ & 13.01 & 17.77 & 17.39 & 8.0 & 393 & 2.685 & 7,160 \\
\hline 70 & $370-420$ & 3.98 & 3.96 & 3.37 & 3.0 & 392 & 0.841 & 702 \\
\hline $7 E$ & $440-500$ & 8.11 & 8.10 & 3.105 & 3.0 & 992 & 0.277 & 76 \\
\hline $9 A$ & $0-50$ & 8.10 & 3.09 & 3.095 & 3.01 & 395 & 0.249 & 62 \\
\hline 98 & $90-105$ & 8.96 & 8.94 & 8.95 & 8.01 & 395 & 0.327 & 680 \\
\hline $9 C$ & $210-270$ & 18.05 & 17.77 & 17.91 & 9.01 & 935 & 2.684 & 7,167 \\
\hline 90 & $430-755$ & 10.54 & 10.49 & 10.515 & 3.01 & 994 & 1.351 & 1,313 \\
\hline $10 \mathrm{~A}$ & $0-60$ & 8.11 & 8.10 & $3.1 \cup 5$ & 8.01 & 994 & 0.263 & 69 \\
\hline $10 \mathrm{~B}$ & $90-150$ & 8.98 & 3.96 & 8.97 & 8.01 & 994 & 0.836 & 695 \\
\hline $10 \mathrm{C}$ & $210-270$ & 10.01 & 9.97 & 9.99 & 8.01 & 994 & 1.201 & 1,433 \\
\hline 100 & $320-380$ & 8.97 & 8.95 & 8.96 & 8.01 & 994 & 0.832 & 683 \\
\hline 1UE & $400-465$ & 8.10 & 8.09 & 8.095 & 3.01 & 994 & 0.249 & 52 \\
\hline
\end{tabular}


TABLE 2. PKL test facility orifice-meter test results. $a$ / (Continued)

\begin{tabular}{|c|c|c|c|c|c|c|c|c|}
\hline \multirow{2}{*}{$\begin{array}{l}\text { Test } \\
\text { no. }\end{array}$} & \multirow{2}{*}{$\begin{array}{c}\text { Time } \\
\text { interval, } \\
\text { s }\end{array}$} & \multicolumn{4}{|c|}{ Orifice-meter signal, mA } & \multirow{2}{*}{$\begin{array}{c}\text { Density } \\
\mathrm{kg} / \mathrm{m}\end{array}$} & \multirow{2}{*}{$\begin{array}{c}\text { Calculated } \\
\text { velocity, } \\
\text { m/s }\end{array}$} & \multirow{2}{*}{$\begin{array}{l}\text { Calculated } \\
\text { monentym, } \\
\mathrm{kg} / \mathrm{m}-\mathrm{s}\end{array}$} \\
\hline & & High & Low & Average & Offset & & & \\
\hline $11 \mathrm{~A}$ & $0-60$ & 8.10 & 8.09 & 8.095 & 8.01 & 997 & 0.248 & 62 \\
\hline $11 B$ & $105-180$ & 8.97 & 8.96 & 8.965 & 8.01 & 996 & 0.833 & 691 \\
\hline $11 \mathrm{C}$ & $220-280$ & 10.03 & 10.00 & 10.015 & 8.01 & 995 & 1.208 & 1,451 \\
\hline 110 & $330-390$ & 8.97 & 8.96 & 8.965 & 8.01 & 995 & 0.834 & 691 \\
\hline $11 \mathrm{E}$ & $410-470$ & 8.11 & 8.10 & 8.105 & 8.01 & 995 & 0.263 & 69 \\
\hline $12 \mathrm{~A}$ & $0-60$ & 8.10 & 8.09 & 8.095 & 8.01 & 995 & 0.249 & 62 \\
\hline $12 \mathrm{~B}$ & $90-150$ & 8.97 & 8.95 & 8.96 & 8.01 & 995 & 0.831 & 688 \\
\hline $12 \mathrm{C}$ & $195-260$ & 10.02 & 10.00 & 10.01 & 8.01 & 995 & 1.206 & 1,448 \\
\hline 120 & $300-360$ & 8.98 & 8.96 & 8.97 & 8.01 & 994 & 0.836 & 695 \\
\hline $12 \mathrm{E}$ & $375-450$ & 8.10 & 8.09 & 8.095 & 8.01 & 994 & 0.249 & 62 \\
\hline $13 A$ & $0-60$ & 8.11 & 8.09 & 8.10 & 8.01 & 996 & 0.256 & 65 \\
\hline $13 B$ & $90-150$ & 8.98 & 3.96 & 8.97 & 8.01 & 995 & 0.836 & 695 \\
\hline $13 C$ & $200-260$ & 10.03 & 9.99 & 10.01 & 8.01 & 994 & 1.207 & 1,448 \\
\hline 130 & $320-380$ & 8.98 & 8.96 & 8.97 & 8.01 & 994 & 0.836 & 695 \\
\hline $13 \mathrm{E}$ & $410-470$ & 8.11 & 8.09 & 8.10 & 8.01 & 994 & 0.256 & 65 \\
\hline $14 A$ & $0-60$ & 8.11 & 8.10 & 8.095 & 8.01 & 994 & 0.249 & 62 \\
\hline $14 B$ & $90-150$ & 8.96 & 8.94 & 8.95 & 8.01 & 993 & 0.828 & 680 \\
\hline $14 \mathrm{C}$ & $215-270$ & 10.02 & 9.98 & 10.00 & 8.01 & 993 & 1.204 & 1,441 \\
\hline 140 & $350-410$ & 8.97 & 8.94 & 8.955 & 8.01 & 993 & 0.830 & 684 \\
\hline $14 \mathrm{E}$ & $425-480$ & 8.11 & 8.09 & 8.10 & 8.01 & 993 & 0.256 & 65 \\
\hline $15 \mathrm{~A}$ & $0-100$ & 10.02 & 9.99 & 10.005 & 8.015 & 993 & 0.616 & 376 \\
\hline $15 B$ & $150-200$ & 8.96 & 8.95 & 8. 955 & 8.015 & 993 & 0.423 & 178 \\
\hline $15 C$ & $290-360$ & 10.04 & 9.97 & 10.005 & 8.015 & 993 & 0.616 & 376 \\
\hline $15 D$ & $420-460$ & 12.44 & 12.35 & 12.395 & 8.015 & 953 & 0.914 & 829 \\
\hline
\end{tabular}

a/ Test results based on calculations of equations (24) and (25).

\section{ORIF ICE-METER RESULTS}

Only one size orifice meter was used to obtain flow rates in the water loop. The velocity of the flow (Vorifice) is detemined from the orifice-meter signal (MA) in $m A$, from the offset (OFFSET) in the instrument in $m A$, and from the fluid density $\left(\rho_{W}\right)$ in $\mathrm{kg} / \mathrm{m}^{3}$ from the following relationship $[8]$ :

$$
v_{\text {orifice }}(\mathrm{m} / \mathrm{s})=\frac{0.1952}{0.00513} \sqrt{\frac{M A-\text { OFFSET }}{2 p}} .
$$


During testing, the orifice-meter signal varied between a high and low value with the average of these signals used in the above equation. The offset usually remained steady at one value. Density was obtained from steam tables based on a measurement of temperature by the spool thermocouple.

Table 2 gives results for the above described calculation for all the acceptance test.s. Al so given in Table 2 are the results of a calculation of momentum flux obtained from:

$$
\text { Momentum } f 1 u x\left(\mathrm{~kg} / \mathrm{m}-\mathrm{s}^{2}\right)=\rho_{W} v_{\text {orifice }}^{2} \text {. }
$$

\section{SPOOL TEST RESULTS}

During each of the acceptance tests, the PKL DAS acquired data and performed calculations at 10-s intervals. The average of the turbine velocity and momentum flux measurements during a test are given in Table 3 . Al so shown and repeated from Table 2 are the velocity and momentum flux results obtained from the PKL water-100p orifice meter. The percentage difference of the readings between the spool-determined and orifice-determined velocities and momentum fluxes is shown in Table 3 and is calculated by:

$$
\% \text { diff }=\frac{Y_{\text {spool }-Y_{\text {orifice }}}}{Y_{\text {orifice }}} \times 100
$$

where

$$
\begin{aligned}
& Y_{\text {spool }}=\text { spool-determined ayerage velocity or momentum flux } \\
& Y_{\text {orifice }}=\text { orifice-detemined ayerage velocity or momentum flux. }
\end{aligned}
$$

Allowable percentage errors in reading due to instrument tolerances of $1 \%$ of full scale are given in the "Allow $\pm_{0}^{\circ}$ column of Table 3 . The tolerance for 
vel ocity measurements is $0.6 \mathrm{~m} / \mathrm{s}$. The tolerance for momentum flux is 72 $\mathrm{kg} / \mathrm{m}-\mathrm{s}^{2}$. The allowable percentage error is calculated by:

$$
\text { Allow }+\%=\frac{\text { TOL }}{\text { orifice }}
$$

where

TOL = spool velocity or momentum flux tolerance.

TABLE 3. PKL spool piece water-flow test results.

\begin{tabular}{|c|c|c|c|c|c|c|c|c|c|c|}
\hline \multirow[b]{2}{*}{$\begin{array}{l}\text { Test } \\
\text { no. }\end{array}$} & \multirow[b]{2}{*}{$\begin{array}{c}\text { Spool } \\
\text { no. }\end{array}$} & \multirow{2}{*}{$\begin{array}{l}\text { Flow } \\
\text { direc- } \\
\text { tion } \\
\text { b/ }\end{array}$} & \multicolumn{4}{|c|}{ Velocity $(\mathrm{m} / \mathrm{s})$} & \multicolumn{4}{|c|}{ Momentum flux $\left(\mathrm{kg} / \mathrm{m}-\mathrm{s}^{2}\right)$} \\
\hline & & & Turbine & Orifice & $\begin{array}{c}\% \text { diff } \\
\text { c/ }\end{array}$ & $\begin{array}{l}\text { Altgw } \\
+\% \underline{\text { d }}\end{array}$ & $\begin{array}{l}\text { Drag } \\
\text { Screen }\end{array}$ & Orifice & $\begin{array}{c}\text { of diff } \\
\text { c/ }\end{array}$ & $\begin{array}{l}\text { A1 } 7 q w \\
+\%=-\end{array}$ \\
\hline $5 A$ & 1 & $F$ & 0.292 & 0.308 & -5.2 & 195.0 & 95 & 94 & 1.1 & 77.0 \\
\hline $5 B$ & 1 & $\mathrm{~F}$ & 0.819 & 0.841 & -2.6 & 71.0 & 686 & 702 & -2.3 & 10.0 \\
\hline $5 C$ & 1 & $\mathrm{~F}$ & 2.615 & 2.681 & -2.5 & 22.0 & 7035 & 7138 & -1.4 & 1.0 \\
\hline 50 & 1 & $F$ & 0.831 & 0.852 & -2.5 & 70.0 & 704 & 724 & -2.8 & 9.9 \\
\hline $5 \mathrm{E}$ & 1 & $\mathrm{~F}$ & 0.245 & 0.277 & -12.0 & 217.0 & 80 & 76 & 5.3 & 95.0 \\
\hline $5 F$ & 1 & $\mathrm{~F}$ & 2.650 & 2.703 & -2.0 & 22.0 & 7279 & 7257 & 0.3 & 1.0 \\
\hline $6 \mathrm{~A}$ & 1 & $\mathrm{~F}$ & 0.278 & 0.263 & 5.7 & 228.0 & 75 & 69 & 8.7 & 104.0 \\
\hline $6 \mathrm{~B}$ & 1 & $\mathrm{~F}$ & 0.821 & 0.839 & -2.1 & 72.0 & 686 & 699 & -1.9 & 10.0 \\
\hline $6 C$ & 1 & $\mathrm{~F}$ & 2.585 & 2.680 & -3.5 & 22.0 & 7104 & 7131 & -0.4 & 1.0 \\
\hline $6 D$ & 1 & $F$ & 0.813 & 0.839 & -3.1 & 72.0 & 681 & 699 & -2.6 & 10.0 \\
\hline $6 \mathrm{E}$ & 1 & : & 0.244 & 0.263 & -7.2 & 228.0 & 79 & 69 & 15.0 & 104.0 \\
\hline $7 A$ & 1 & $\mathbf{R}$ & 0.247 & 0.277 & -11.0 & 217.0 & 131 & 76 & 72.0 & 95.0 \\
\hline $7 B$ & 1 & $R$ & 0.844 & 0.841 & 0.4 & 71.0 & 679 & 702 & -3.3 & 10.0 \\
\hline $7 \mathrm{C}$ & 1 & R & 2.726 & 2.685 & 1.5 & 22.0 & 6860 & 7160 & -4.2 & 1.0 \\
\hline $7 D$ & 1 & $\mathrm{R}$ & 0.831 & 0.841 & -1.2 & 71.0 & 693 & 702 & -1.3 & 10.0 \\
\hline $7 E$ & 1 & $\mathrm{R}$ & 0.255 & 0.277 & -7.9 & 217.0 & 179 & 76 & 135.0 & 95.0 \\
\hline $9 A$ & 4 & $\mathrm{R}$ & 0.146 & 0.249 & -41.0 & 241.0 & 58 & 62 & 6.5 & 116.0 \\
\hline $9 B$ & 4 & $\mathrm{R}$ & 0.761 & 0.827 & -8.0 & 73.0 & 675 & 680 & 0.7 & 11.0 \\
\hline $9 C$ & 4 & $\mathrm{R}$ & 2.605 & 2.684 & -2.9 & 22.0 & 7857 & 7167 & 9.6 & 1.0 \\
\hline 9D & 4 & $R$ & 1.277 & 1.351 & -5.5 & 44.0 & 1871 & 1813 & 3.2 & 4.0 \\
\hline $10 A$ & 4 & $\mathrm{R}$ & 0.157 & 0.263 & -40.0 & 228.0 & 62 & 69 & 10.0 & 104.0 \\
\hline $10 \mathrm{~B}$ & 4 & $\mathrm{R}$ & 0.761 & 0.836 & -9.0 & 72.0 & 697 & 695 & 0.3 & 10.0 \\
\hline $10 \mathrm{C}$ & 4 & $\mathrm{R}$ & 1.090 & 1.201 & -9.2 & 50.0 & 1469 & 1433 & 2.5 & 5.0 \\
\hline 10D & 4 & $\mathrm{R}$ & 0.761 & 0.832 & -8.5 & 72.0 & 688 & 688 & 0.0 & 0.0 \\
\hline $10 E$ & 4 & $\mathrm{R}$ & 0.136 & 0.249 & -45.0 & 241.0 & 58 & 62 & 6.5 & 116.0 \\
\hline
\end{tabular}


Table 3. PKL spool piece water-flow test results.a/ (Continued)

\begin{tabular}{|c|c|c|c|c|c|c|c|c|c|c|}
\hline \multirow[b]{2}{*}{$\begin{array}{l}\text { Test } \\
\text { no. }\end{array}$} & \multirow[b]{2}{*}{$\begin{array}{c}\text { Spool } \\
\text { no. }\end{array}$} & \multirow{2}{*}{$\begin{array}{l}\text { Flow } \\
\text { direc- } \\
\text { tion } \\
\text { b/ }\end{array}$} & \multicolumn{4}{|c|}{ Velocity $(\mathrm{m} / \mathrm{s})$} & \multicolumn{4}{|c|}{ Momentum flux $\left(\mathrm{kg} / \mathrm{m}-\mathrm{s}^{2}\right)$} \\
\hline & & & Turbine & Urifice & 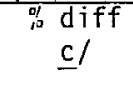 & $\begin{array}{l}\text { Al law } \\
\pm \%=6\end{array}$ & $\begin{array}{l}\text { Drag } \\
\text { Screen }\end{array}$ & Orifice & 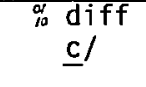 & $\begin{array}{l}\text { A1 low } \\
+x_{-\alpha}^{\prime \prime}\end{array}$ \\
\hline $11 \mathrm{~A}$ & 2 & $\mathrm{~F}$ & 0.130 & 0.248 & -48.0 & 242.0 & 23 & 62 & -63.0 & 116.6 \\
\hline $11 B$ & 2 & $\mathrm{~F}$ & 0.739 & 0.833 & -11.0 & 72.0 & 589 & 691 & -15.0 & 10.0 \\
\hline $11 \mathrm{C}$ & 2 & $\mathrm{~F}$ & 1.122 & 1.208 & -7.1 & 50.0 & 1246 & 1451 & -14.0 & 5.0 \\
\hline 110 & 2 & $\mathrm{~F}$ & 0.738 & 0.834 & -12.0 & 72.0 & 592 & 591 & -14.0 & 10.0 \\
\hline $11 \mathrm{E}$ & 2 & $\mathrm{~F}$ & 0.142 & 0.263 & -46.0 & 228.0 & 31 & 69 & -55.0 & 104.0 \\
\hline $12 A$ & 2 & $\mathrm{~F}$ & 0.142 & 0.249 & -43.0 & 241.0 & 32 & 62 & -48.0 & 116.0 \\
\hline $12 B$ & 2 & $\mathrm{~F}$ & 0.735 & 0.831 & -12.0 & 72.0 & 593 & 688 & -14.0 & 10.0 \\
\hline $12 \mathrm{C}$ & 2 & $\mathrm{~F}$ & 1.123 & 1.206 & -6.9 & 50.0 & 1254 & 1448 & -13.0 & 5.0 \\
\hline 120 & 2 & $\mathrm{~F}$ & 0.748 & 0.836 & -11.0 & 72.0 & 591 & 695 & 0.6 & 10.0 \\
\hline $12 \mathrm{E}$ & 2 & $\mathrm{~F}$ & 0.144 & 0.249 & -42.0 & 241.0 & 32 & 62 & -48.0 & 116.0 \\
\hline $13 A$ & 2 & $\mathrm{R}$ & 0.357 & 0.256 & 39.0 & 234.0 & 99 & 65 & 52.0 & 111.0 \\
\hline $13 B$ & 2 & $\hat{k}$ & 0.937 & 0.836 & 12.0 & 72.0 & 708 & 695 & 1.9 & 10.0 \\
\hline $13 \mathrm{C}$ & 2 & $\mathrm{R}$ & 1.284 & 1.207 & 6.5 & 50.0 & 1529 & 1448 & 5.6 & 5.0 \\
\hline 130 & 2 & $\mathrm{R}$ & 0.924 & 0.836 & 11.0 & 72.0 & 709 & 695 & 2.0 & 10.0 \\
\hline $13 E$ & 2 & $\mathrm{R}$ & 0.357 & 0.256 & 39.0 & 234.0 & 108 & 65 & $6 r .0$ & 111.0 \\
\hline $14 \mathrm{~A}$ & 2 & $\mathrm{R}$ & 0.347 & 0.249 & 39.0 & 241.0 & 108 & 62 & 74.0 & 116.0 \\
\hline $14 B$ & 2 & $\mathrm{R}$ & 0.920 & 0.828 & 11.0 & 72.0 & 697 & 680 & 2.5 & 11.0 \\
\hline $14 \mathrm{C}$ & 2 & $\mathrm{R}$ & 1.300 & 1.204 & 8.0 & 50.0 & 1501 & 1441 & 4.2 & 5.0 \\
\hline 140 & 2 & $\mathrm{R}$ & 0.920 & 0.830 & 11.0 & 72.0 & 698 & 684 & 2.0 & 11.0 \\
\hline $14 \mathrm{E}$ & 2 & $\mathrm{R}$ & 0.357 & 0.256 & 39.0 & 234.0 & 110 & 65 & 69.0 & 111.0 \\
\hline $15 \mathrm{~A}$ & 3 & $\mathrm{~F}$ & 0.631 & 0.616 & 2.4 & 97.0 & 325 & 376 & -14.0 & 19.0 \\
\hline $15 B$ & 3 & $\mathrm{~F}$ & 0.420 & 0.423 & 0.7 & 142.0 & 139 & 178 & -22.0 & 40.0 \\
\hline 150 & 3 & $\mathrm{~F}$ & 0.630 & 0.616 & 2.3 & 97.0 & 325 & 376 & -14.0 & 19.0 \\
\hline $15 D$ & 3 & $\mathrm{~F}$ & 0.916 & 0.914 & 0.2 & 66.0 & 769 & 829 & -7.2 & 8.7 \\
\hline
\end{tabular}

a/ Test results based on calculations of equations (24) and (25).

$\underline{b} / F$ is forward flow, and $R$ is reverse flow.

C ${ }_{\%}$ diff is the percentage difference of the readings between the spool-determined and orifice-determined velocities and momentum fluxes.

d/Allow $+\%$ is the allowable percentage reading errors due to instrument tolerances of $1 \%$ of full scale. 
The perforinance of the turbine flowmeter during acceptance tests was well within the allowable tolerance limits for all of the tests. This is shown in Table 3 by comparing the percentage of reading differences column ( $\%$ diff) and the allowable percentage difference column (Al low $\underline{\alpha}_{2}$ ).

The performance of the drag screen in measuring momentum flux is generally within allowable tolerances. Sone tests exceed tolerances; however, considering the possible error of the orifice meter, the results appear reasonable. For test no. $9 C$, a $9.6 \%$ difference is observed versus an allowable $1 \%$ difference and is an indication of a possible bad data point. 


\section{REFERENCES}

(1) W. Stein, Measurements, Error Analysis, and Calculations of Water and Stean Individual Mass Flow Rates, Velocities, and Related Flow Parameters Obtained From Single-Phase and Two-Phase Prototype Tests of the PKL Instrumented Spool Pieces for the U. S. NRC-RSR 3-D Program, Lawrence Livermore Laboratory, Liverinore, Calif., UCRL-52717 (September 1979).

(2) R. F. Smellie, PKL Instrumented Spool Piece Final Design Review, EG\&G, Inc., San Ramon Operations, MES-014, Tab 2, p. 35 (April 1979).

(3) G. D. Lassahn, Loft Experimental Measurements Uncertainties Analyses. Volume 16: Loft Three-Beam Gamina Densitometer System, Tree-NUREG-1089 (February 1978).

(4) G. D. Lassahn, Loft Three-Beam Densitometer Data Interpretation, Tree-NUREG-1111 (0ctober 1977).

(5) Calculations developed by J. Colson, INEL, Idaho Falls, Idaho; private communication W. Stein/J. Colson (January 1973).

(6) H. Estranda, Jr., and J. D. Sheppard, Some Aspects of Interpreting Two-Phase Flow Measurements in Instrumented Piping Spool Pieces, NUREG-0280 (June 1977).

(7) W. Stein, Integral Acceptance Test Plan for 80.8-mm Horizontal (Type A), 80.8-mm Vertical (Type AV), and 113-mm Horizontal (Type B) PKL Instrumented Spool Piece Systems, EG\&G, Inc., San Ramon Operations, MES-010, Revision A (May 1979). 
APPENDIX A

PKL SPOOL PIECE DRAWINGS

(EG\&G DRAWING NOS. 137086, 137087, AND 137088) 


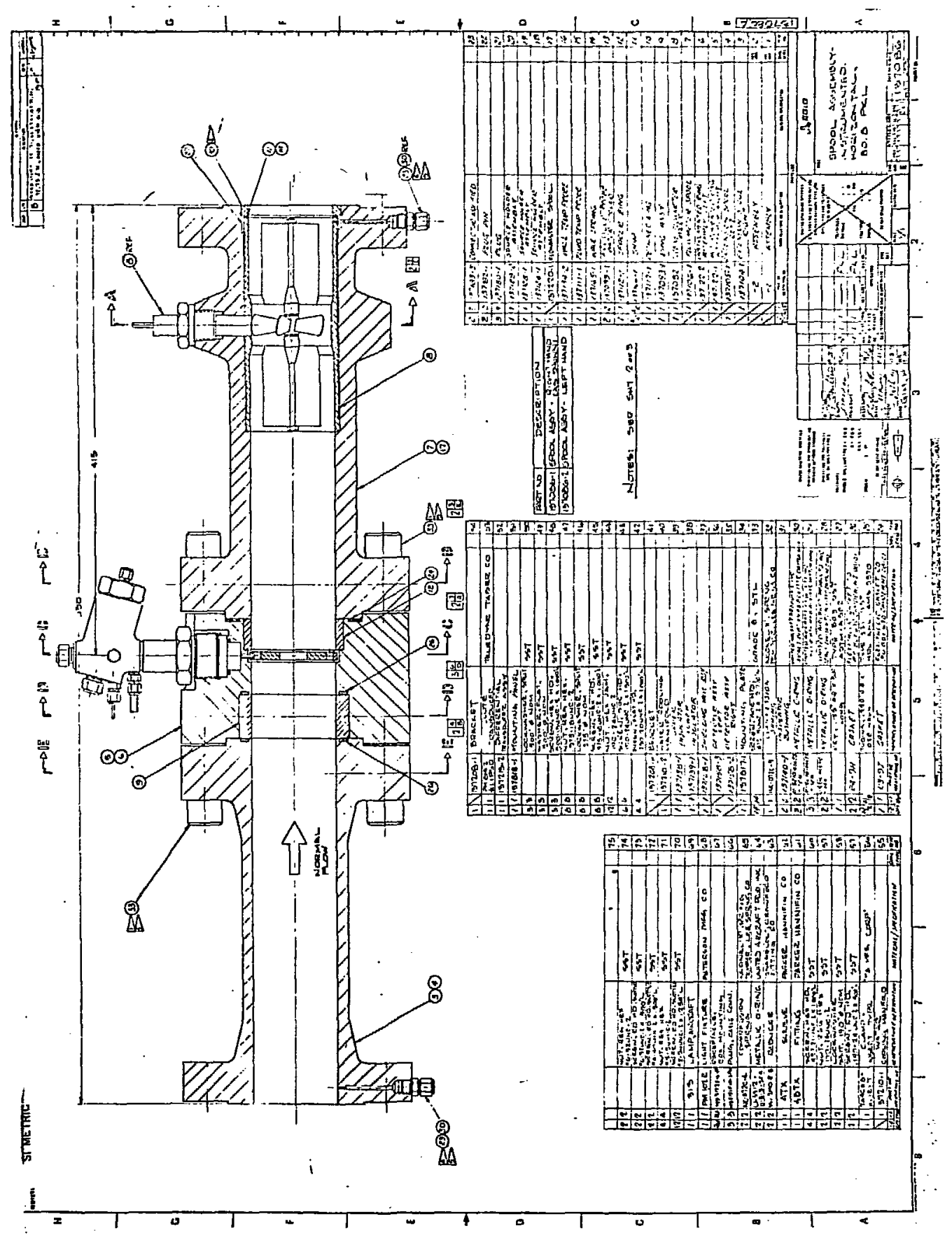




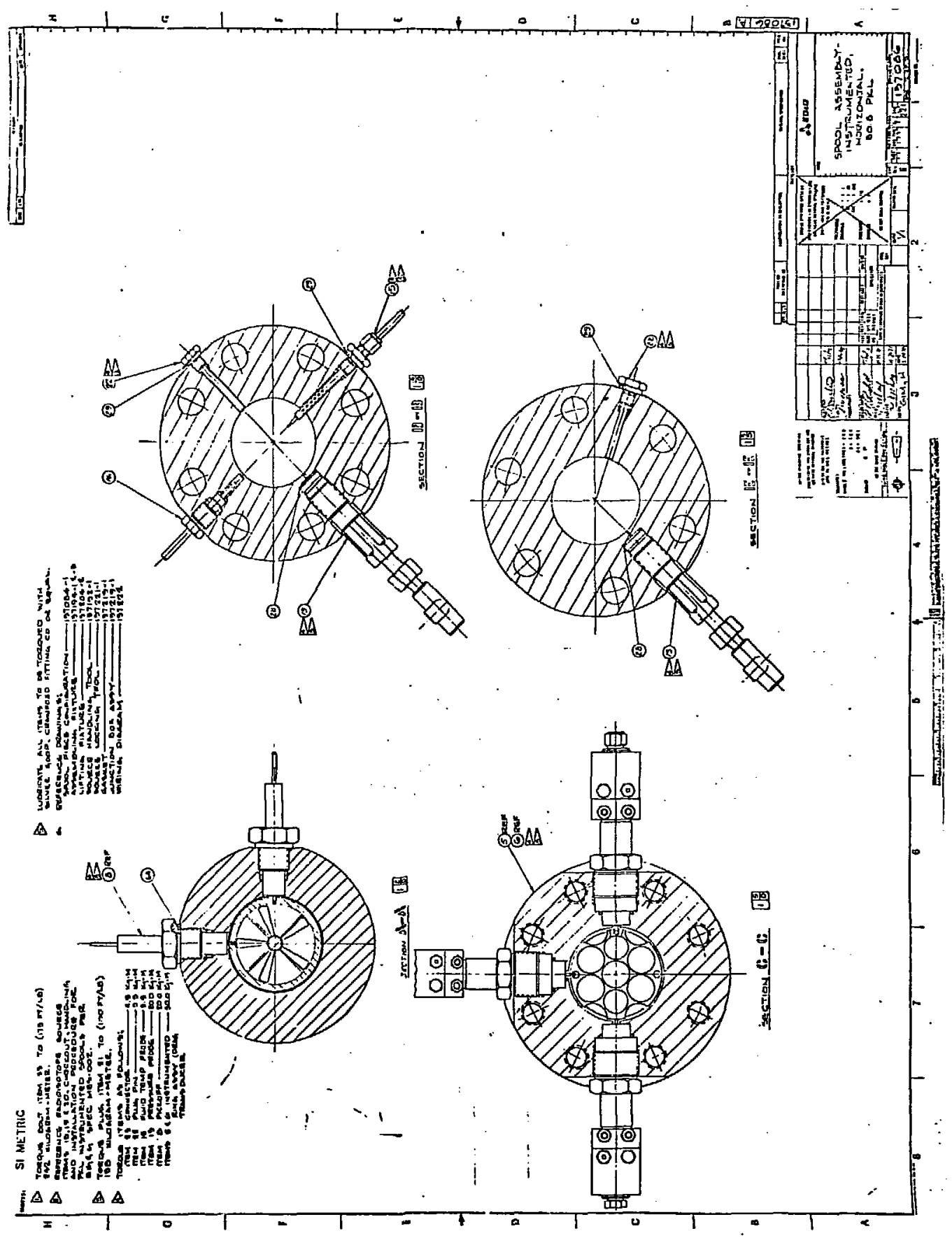




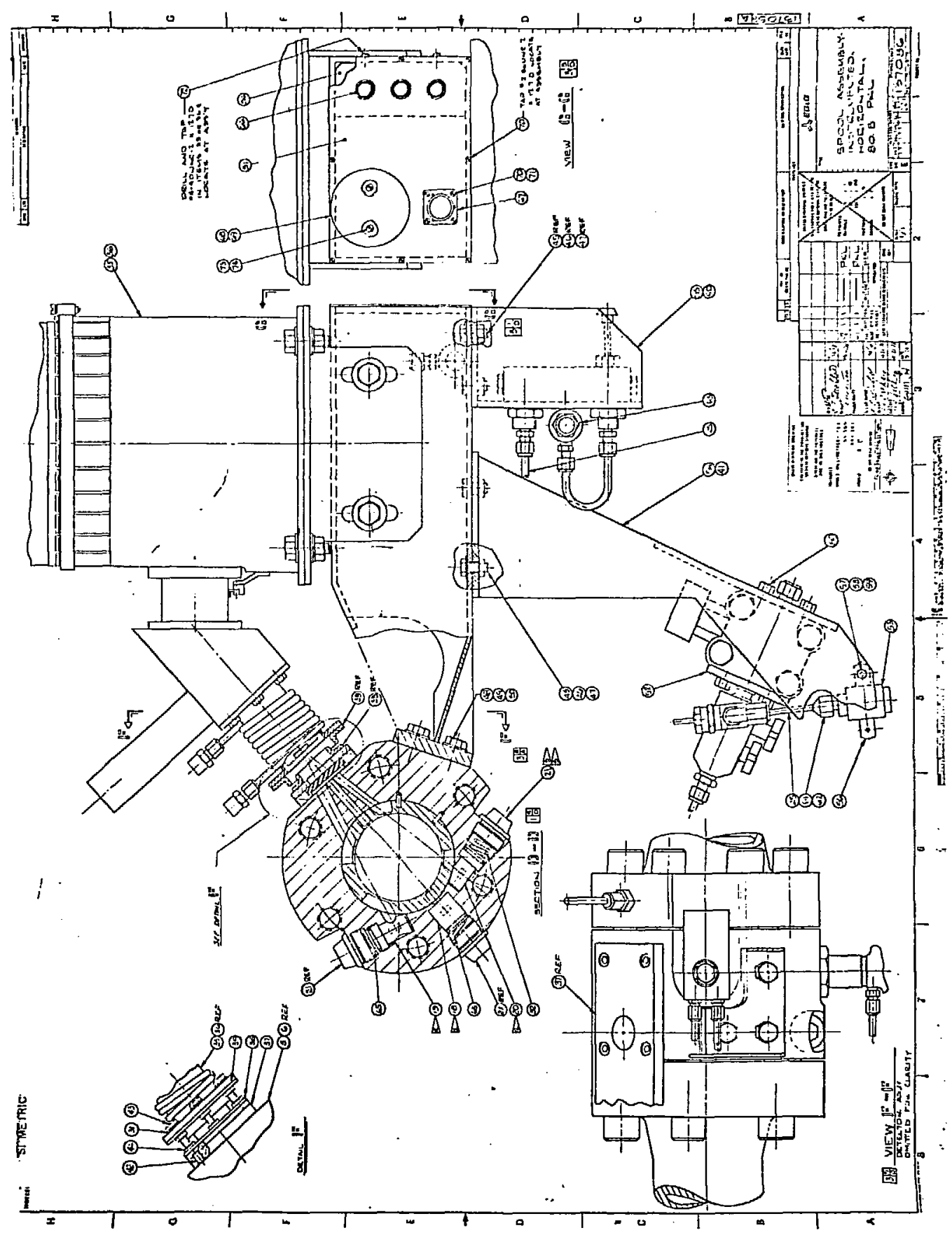




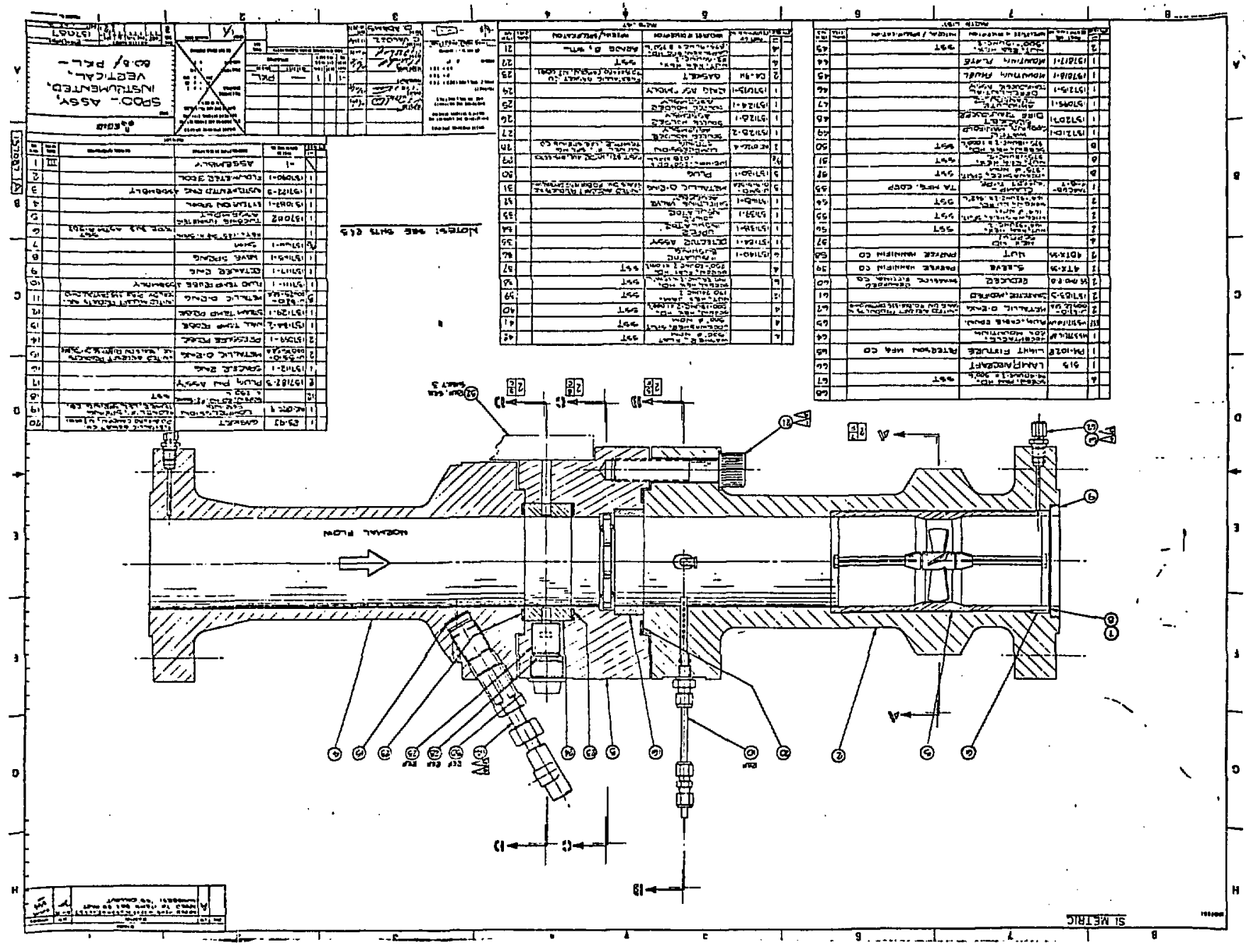




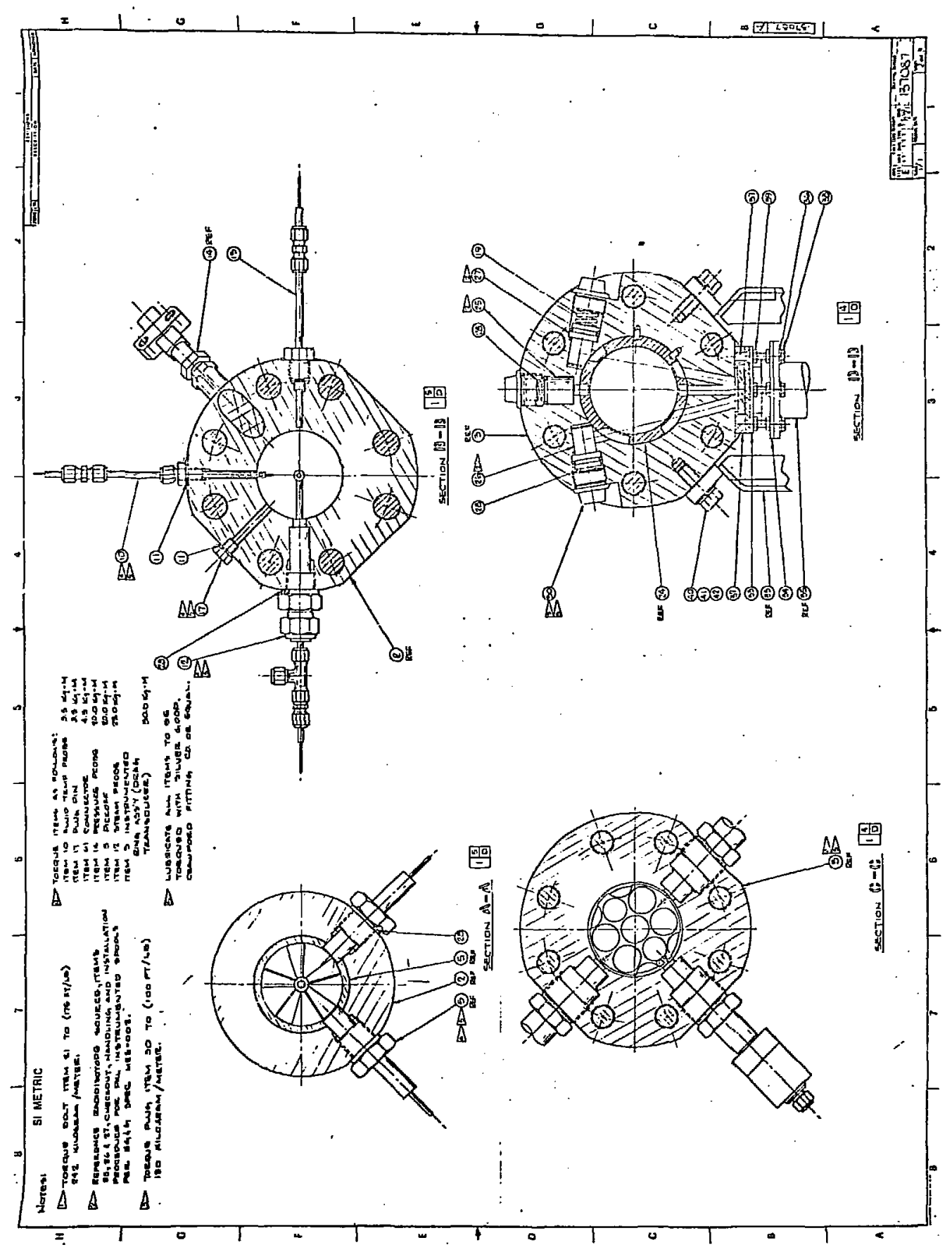




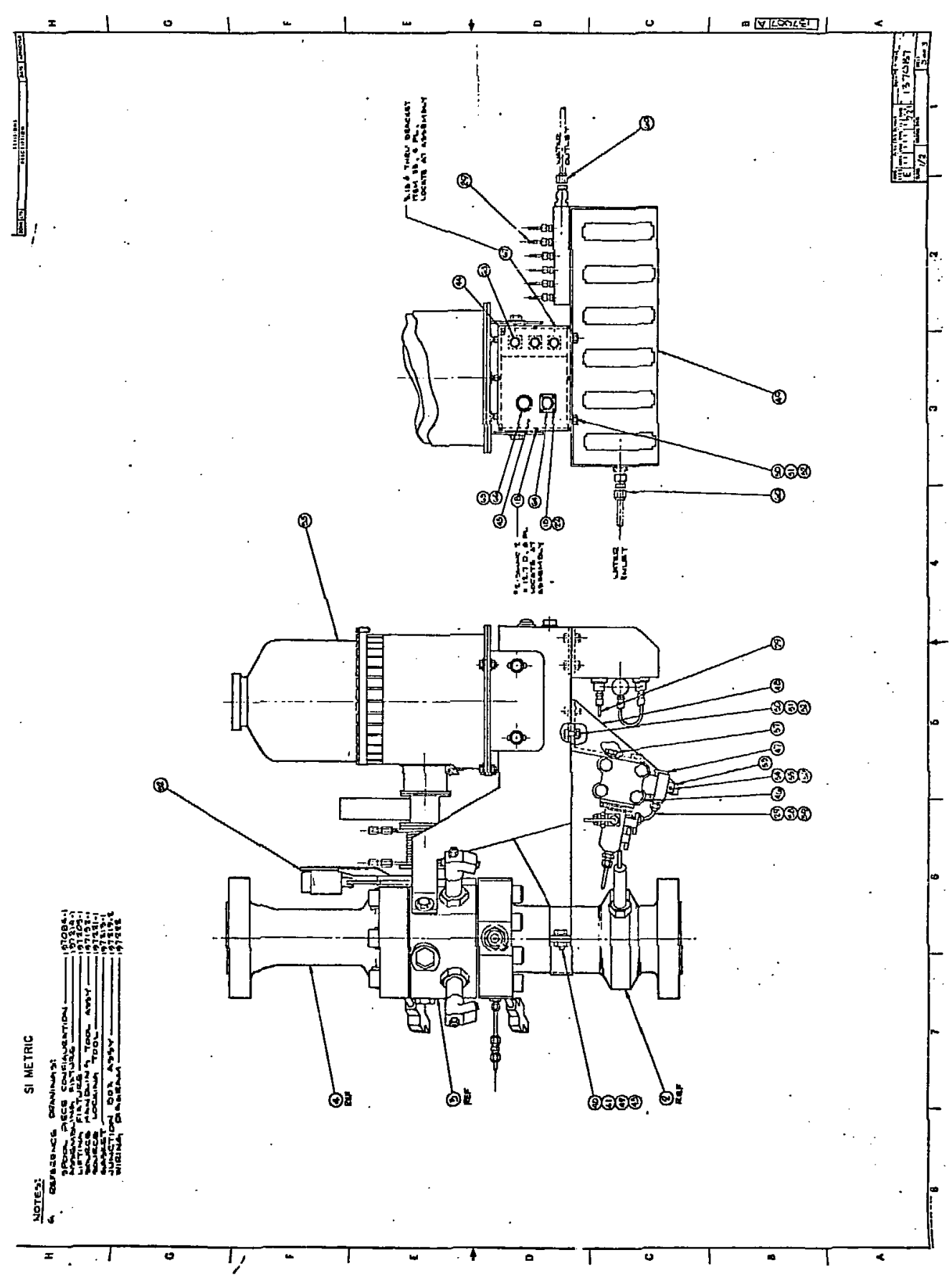




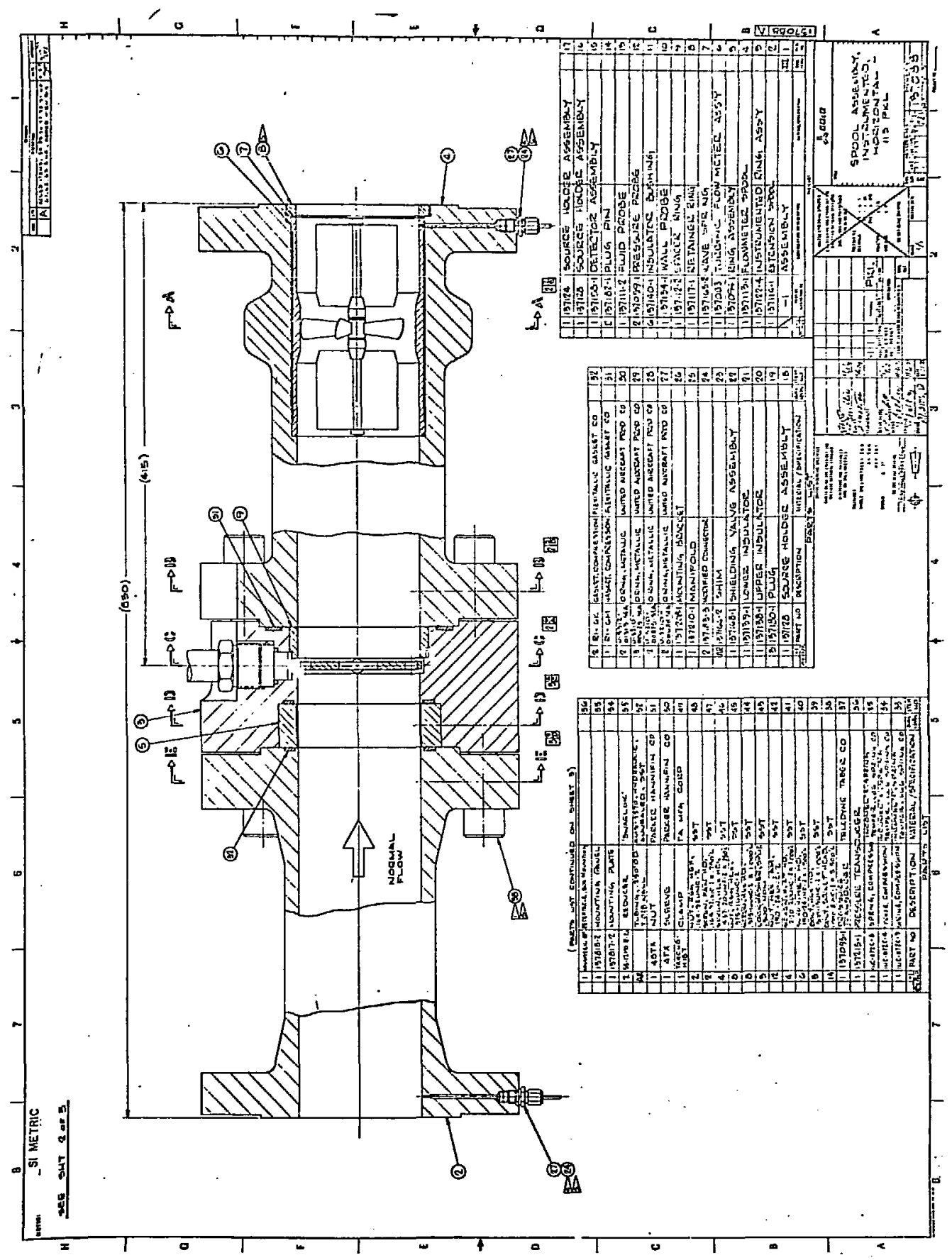




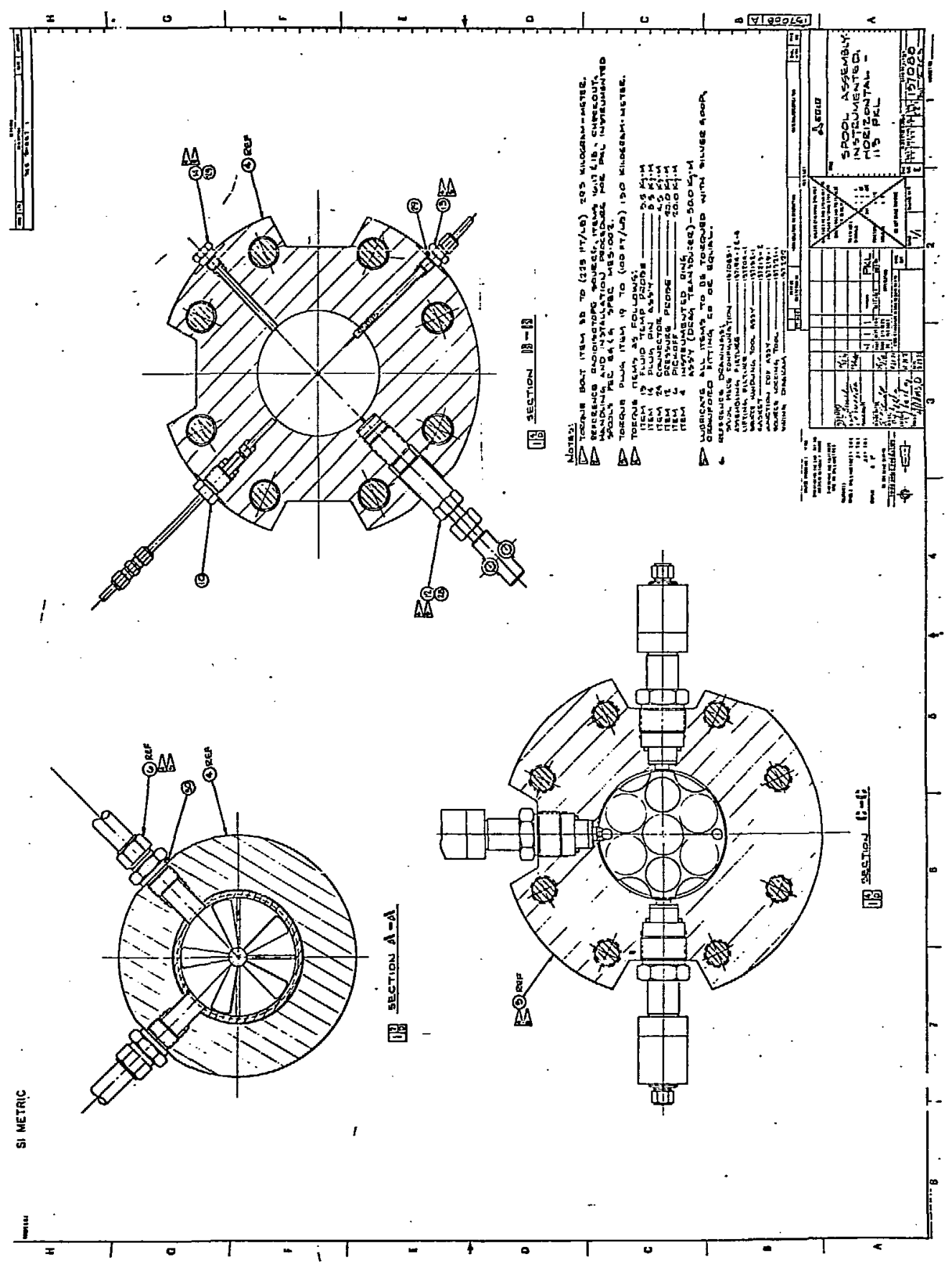




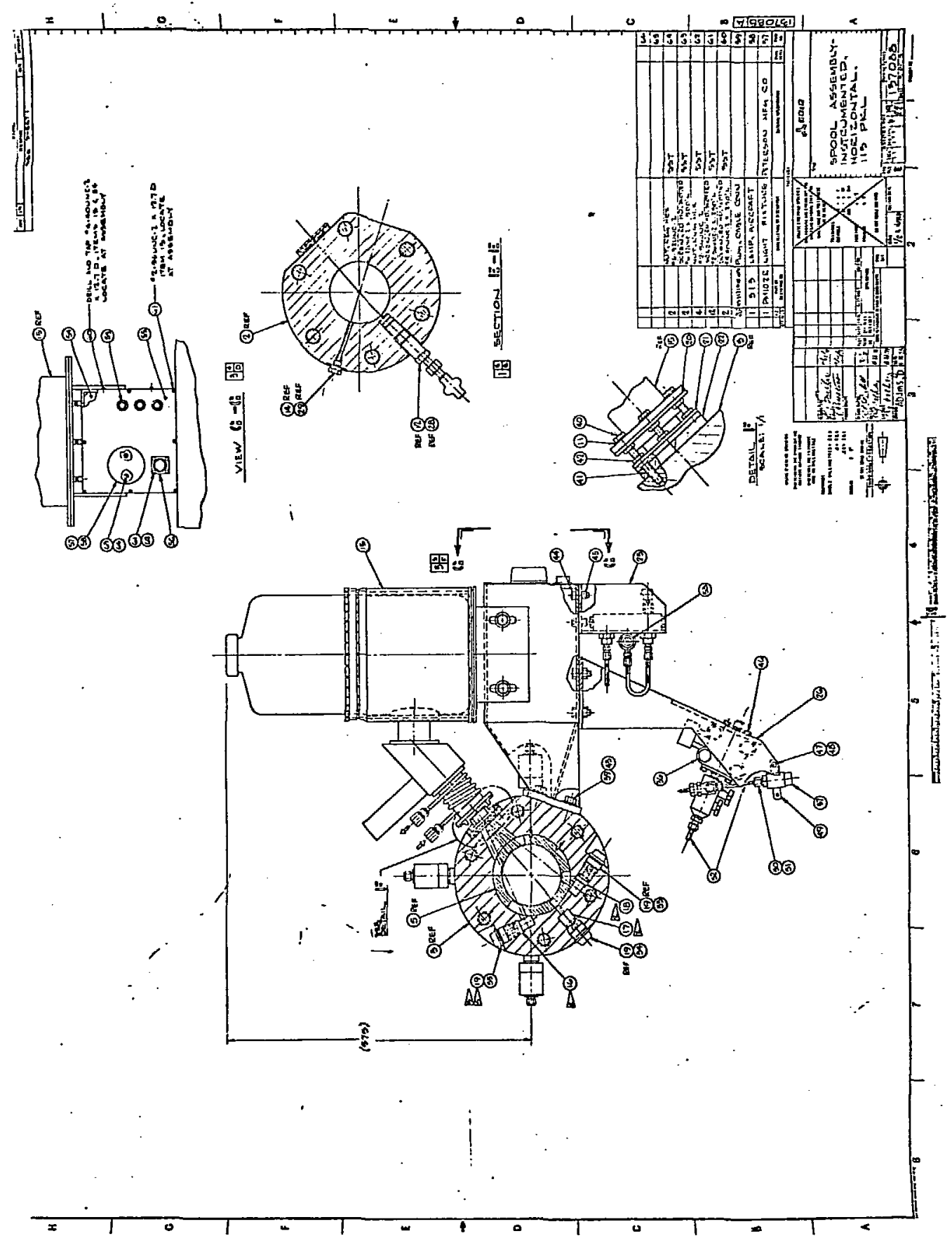


APPENDIX B

PKL SPOOL PIECE TEST RESULTS DATA 
APPENDIX B

Data obtained from PKL acceptance tests are presented in this appendix. For each of the tests, one spool was tested at a time and the data channels were scanned at $10-\mathrm{s}$ intervals.

The date of each test is shown in the heading of the data printout. The test sequence number is given in the heading for each test and identified as "RUN N0." Al so identified and repeated for each $10-\mathrm{s}$ interval is the number of the spool tested (i.e., 1, 2, 3, or 4). Two lines of data are printed out by the DAS for each 10-s interval starting at time equal to zero. The time in seconds from the start of the test is printed at the left of each data 1 ine. The first 1 ine of data presents primarily engineering unit data and the second line presents primarily calculated parameters. Headings for the data in each line are printed out only for the initial set of data lines. For subsequent $10-\mathrm{s}$ intervals, the letters $E$ or $A$ are printed to the left and right of each data line. The letter $E$ identifies the line containing primarily engineering unit data. The letter A identifies the line containing calculated parameters.

Identification of abbreviations and symbols used in the printout is given below:

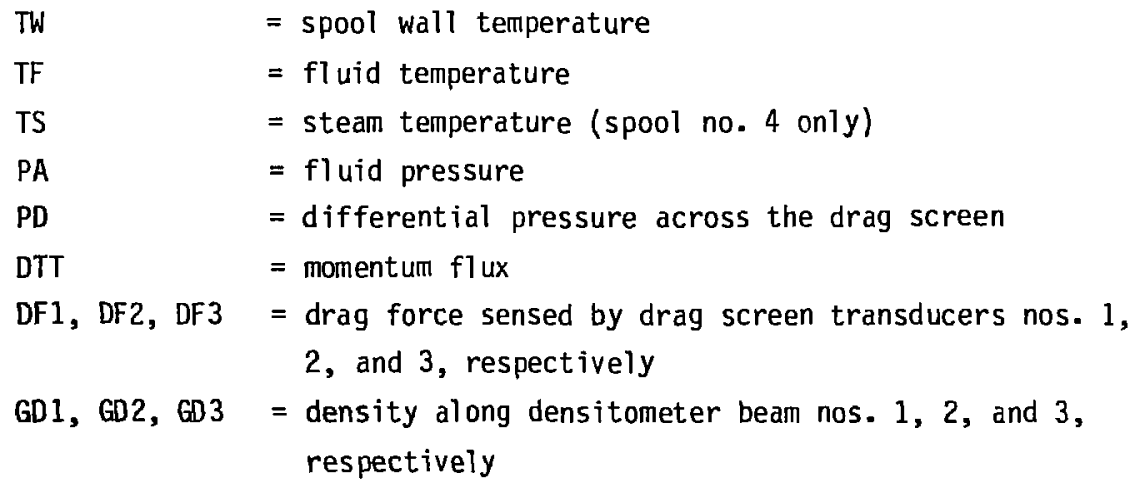




$\begin{array}{ll}\text { FTW } & =\text { turbine neter velocity obtained from a turbine water } \\ & \text { calibration } \\ = & \text { turbine meter velocity obtained from a turbine air } \\ & \text { calibration } \\ \text { FTA } & \text { average pipe cross sectional density obtained from } \\ & \text { densitometer beam readings } \\ = & \text { fluid void fraction } \\ \text { RHOF } & \text { total water mass flow rate based on a calculation } \\ \text { VOID } & \text { using vaiues of RHOF and FTW for density and velocity } \\ \text { MAFT }= & \text { density of the water phase } \\ \text { WATER-DENS }= & \text { water velocity based on equation (20) } \\ \text { WATER-VEL }= & \text { water total mass flow rate based on equation (19) } \\ \text { WATER-MAFW }= & \end{array}$

In calculations of the average pipe cross-sectional density (RHOF) in subcooled water flows, the readings from densitorneter beam no. 3 (DB3) are not used. The upper range of density that this beam can measure is $44.3 \mathrm{~kg} / \mathrm{m}$, and this value is printed out whenever densities along this beam exceed its upper range.

For tests involving spool nos. 2 and 3 , the densitometer was not in operation due to an equipment failure and the data presented represents values due to a dummy input signal.

WS/ar/LSM \#5 


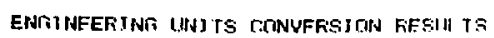

DATE 27-JULN-TO RIIN NN. 5

\begin{tabular}{|c|c|c|c|c|c|c|c|c|c|c|c|c|c|}
\hline $\begin{array}{l}\text { TIME } \\
\text { (SEC) }\end{array}$ & TW, & 13. $\begin{array}{l}\text { TF } \\
\text { CEI_E }\end{array}$ & $s)^{T S}$ & $\begin{array}{l}F A \\
\text { (KFA) }\end{array}$ & $\begin{array}{c}\text { FII } \\
\text { (KFFiti }\end{array}$ & 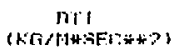 & IIFI & 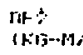 & 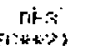 & ind & (i)1: & $\begin{array}{l}\text { Gres } \\
\text { Mimisi }\end{array}$ & $\mathrm{FI}$ กी \\
\hline 0. OOE & 29.13 & 31.75 & 0.00 & 1.85. 76 & 0.000 & 9а.; & $0, \alpha \geqslant 7$ & $0.00 \%$ & $-0 . \cos$ & 1001.100 & $\because A, A+1$ & 44. הNDEF & \\
\hline
\end{tabular}

\section{ANALYSIS MF DATA}

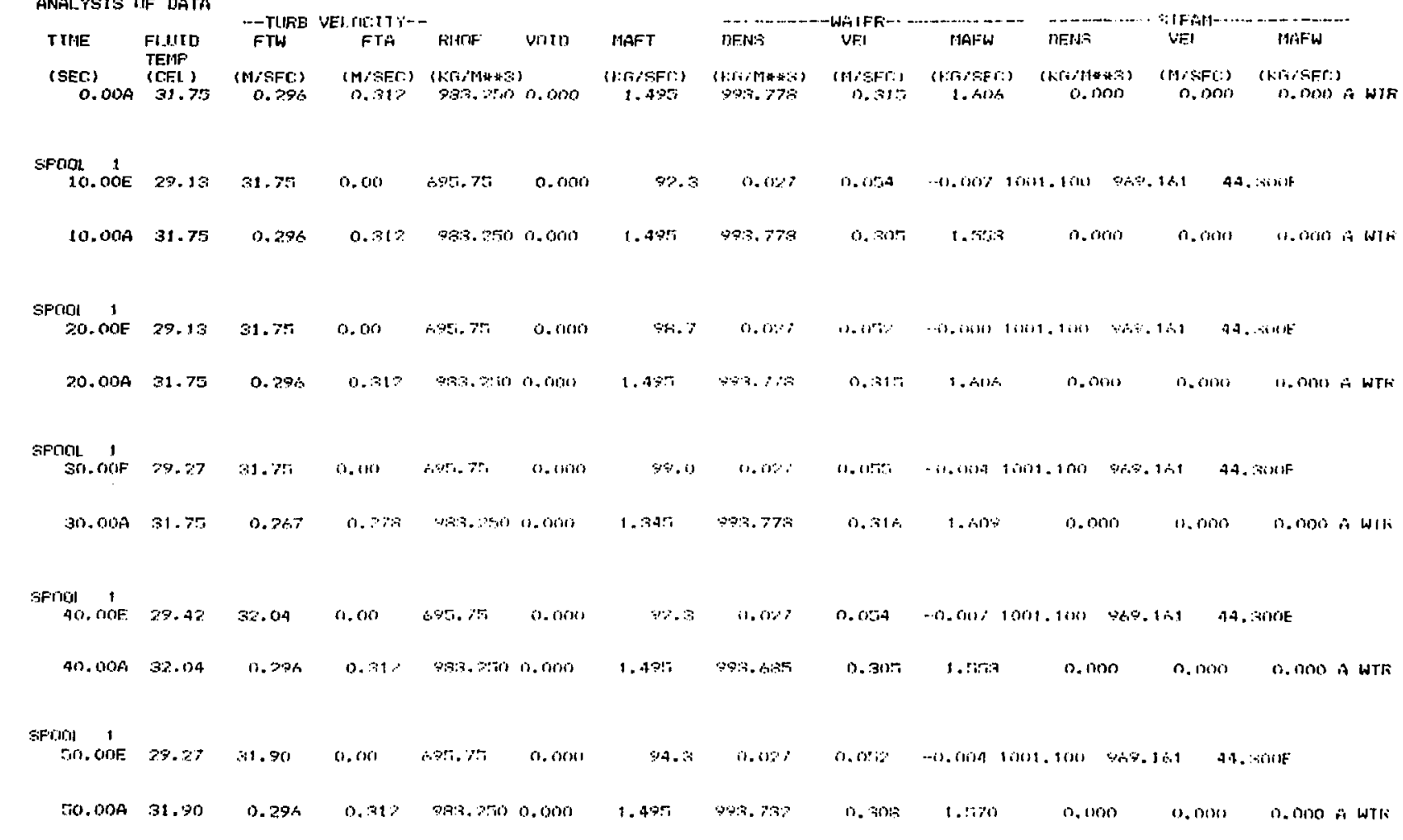




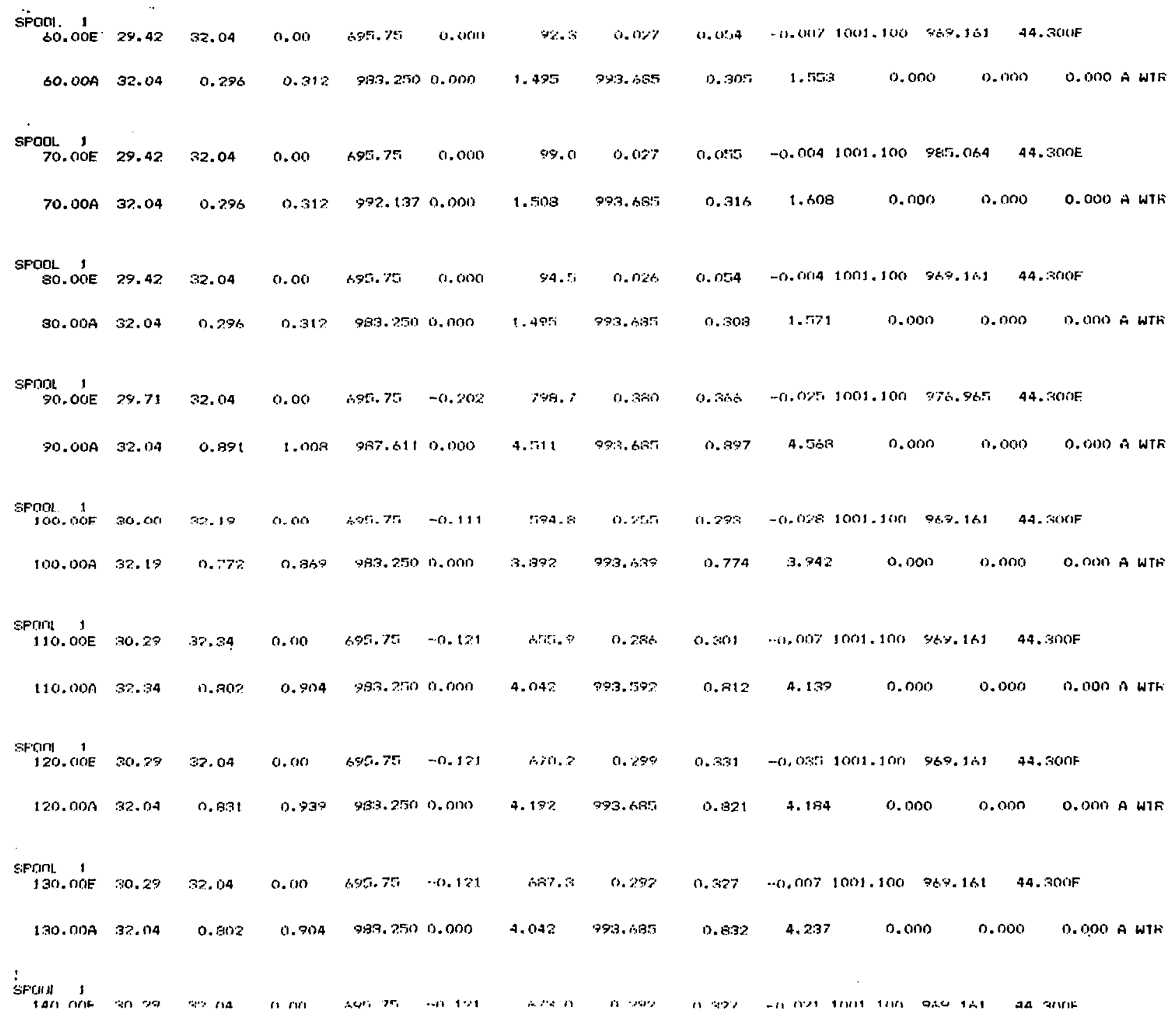




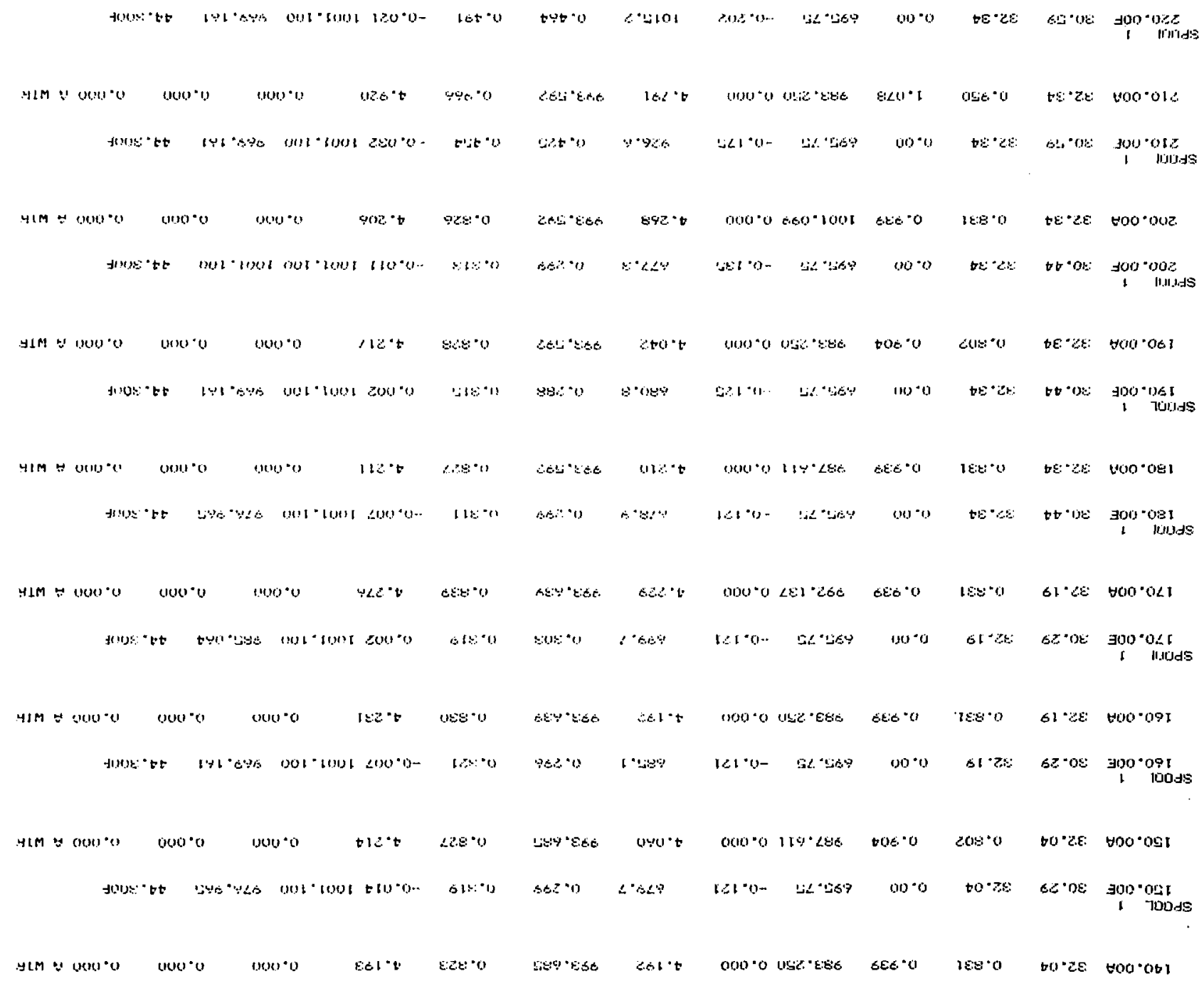




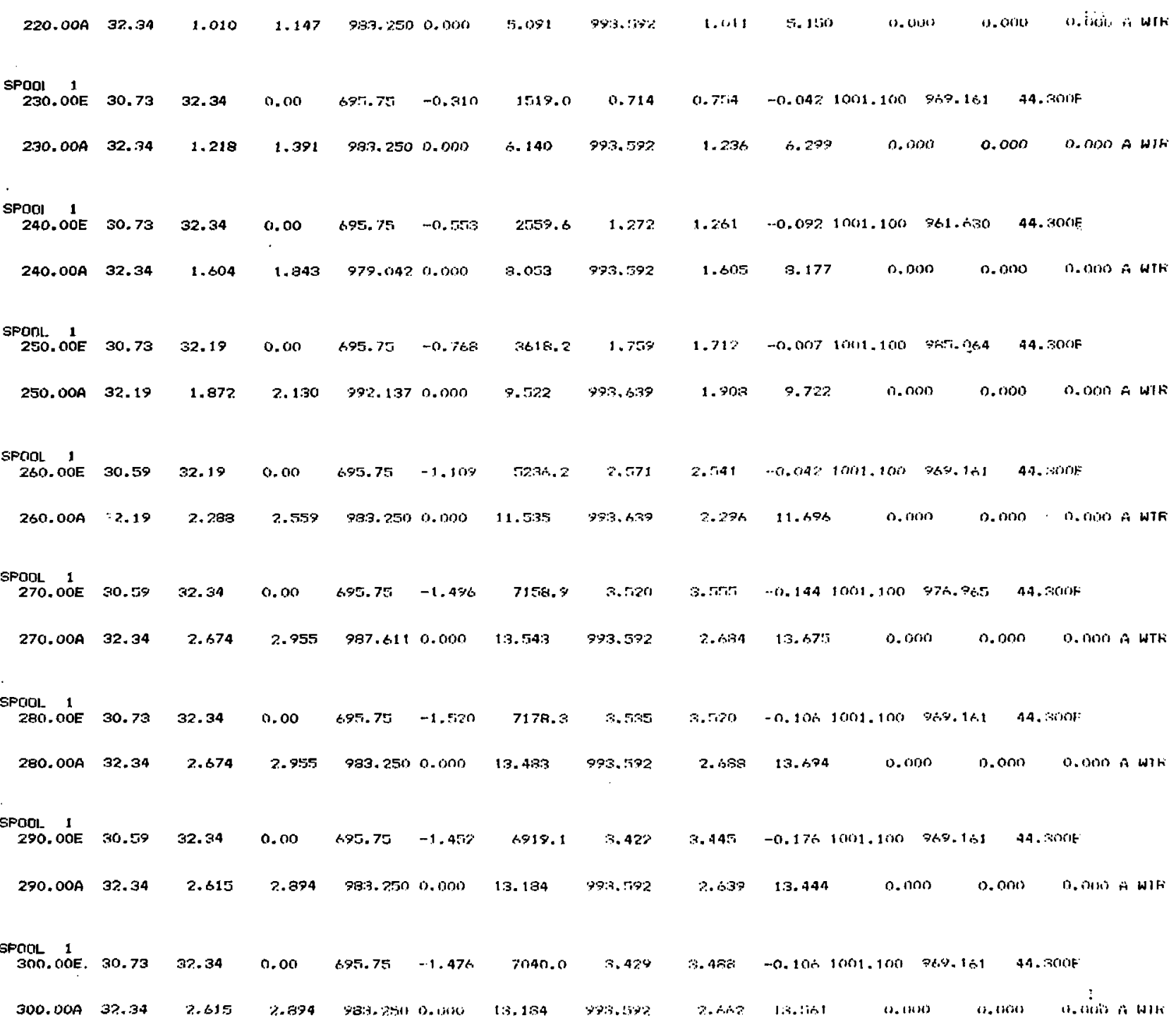




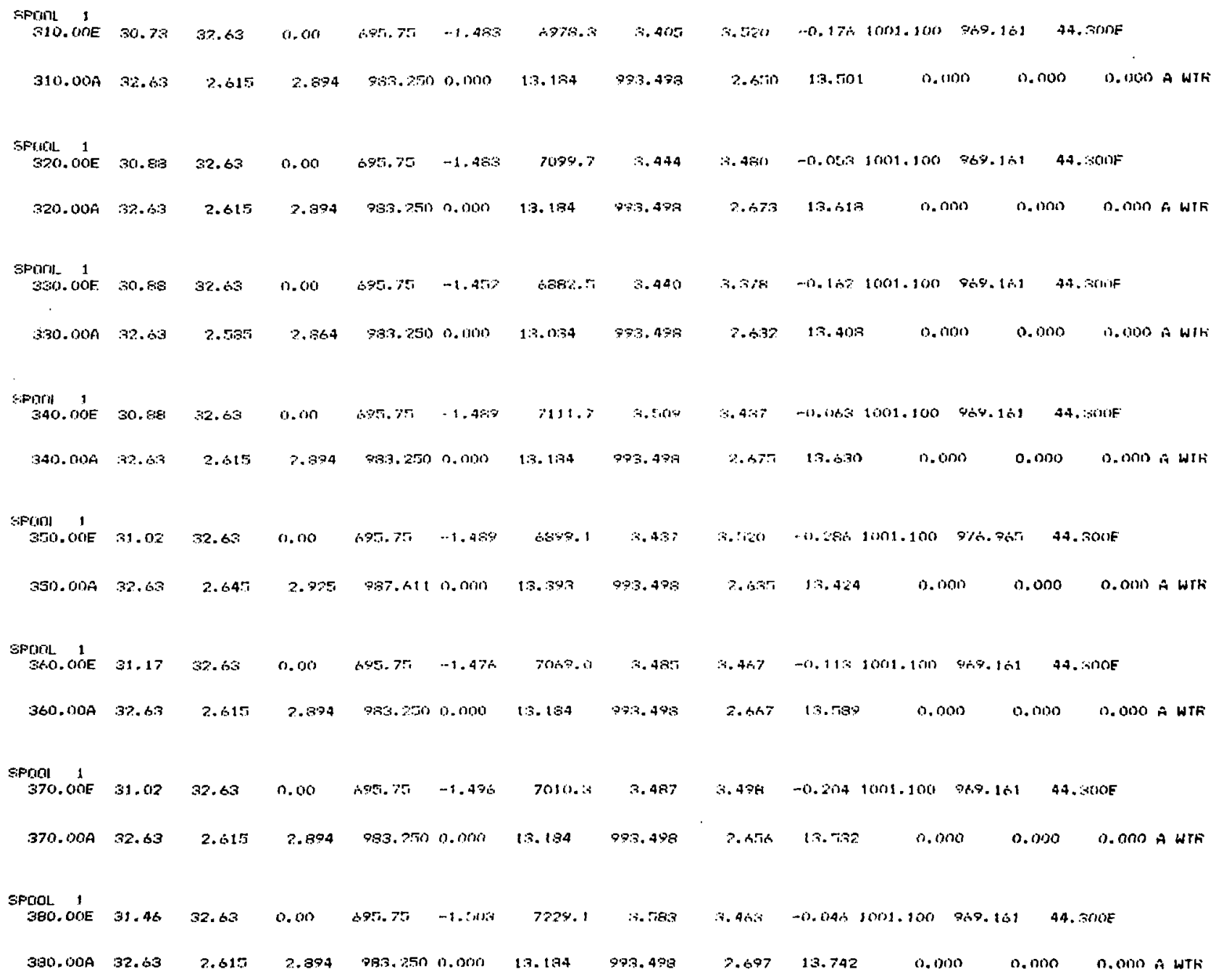




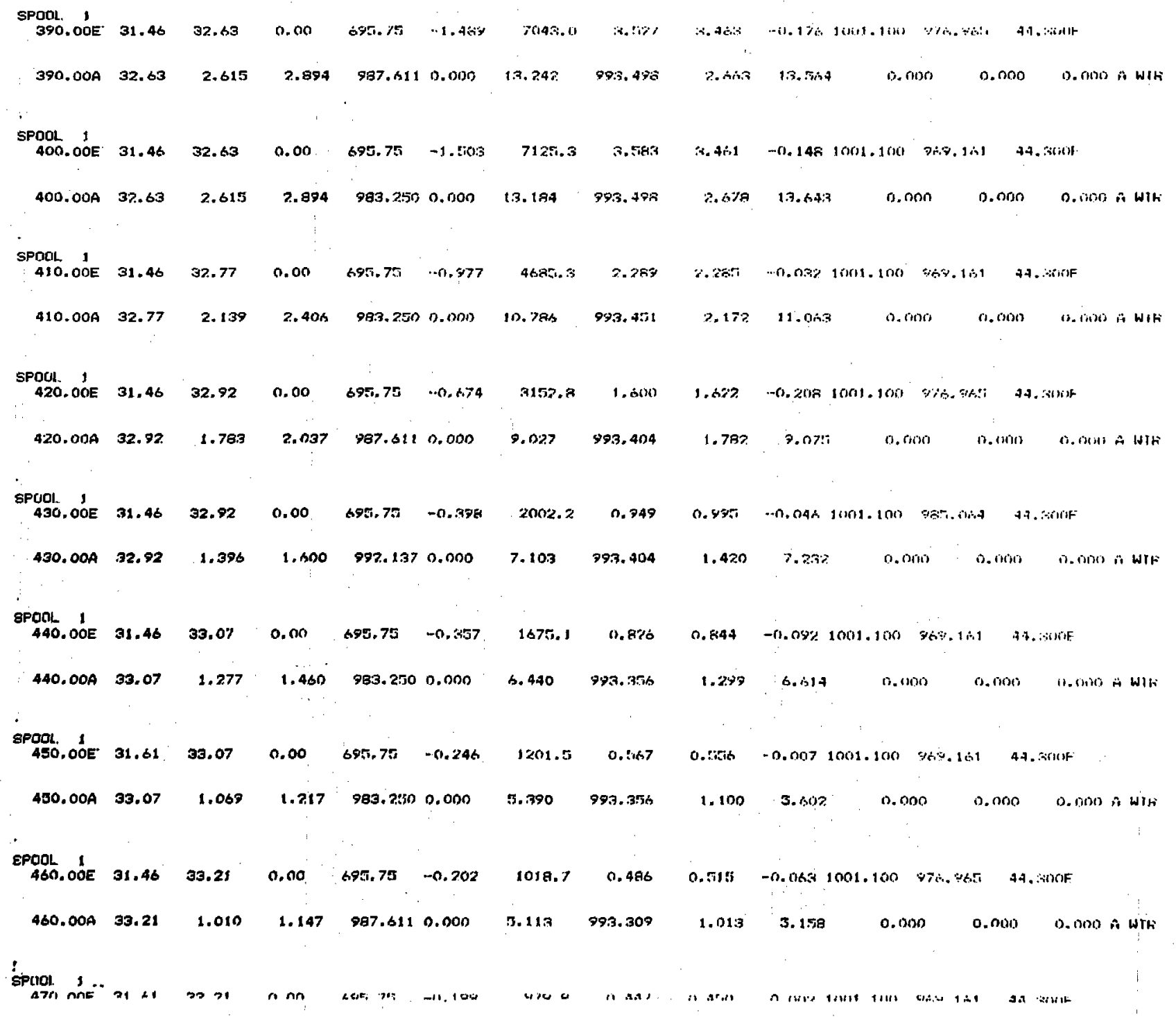




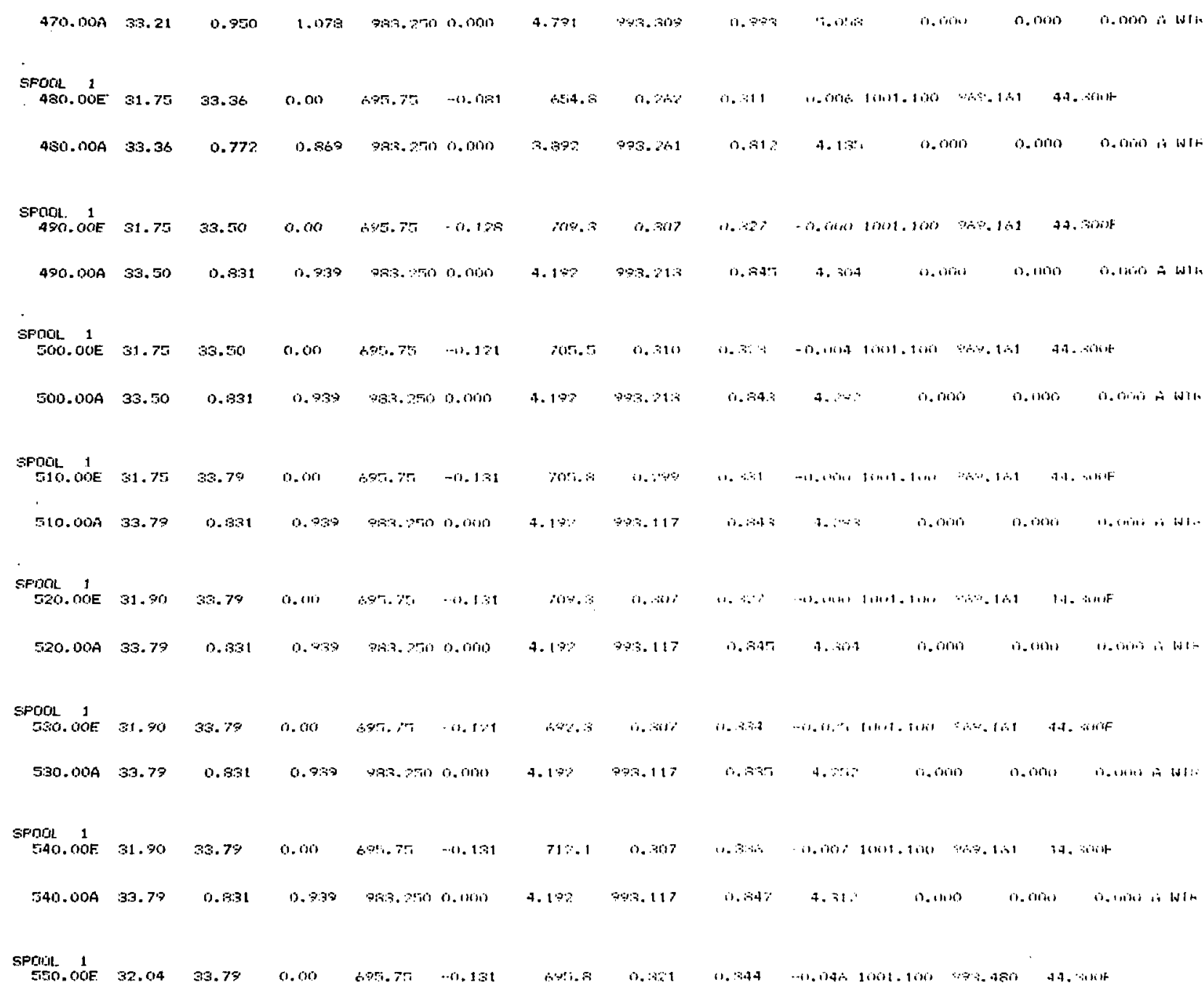




\section{$550.00 \mathrm{~A} \quad 33.79$}

o. 531

0. P:3

SPROL 1

$560.004 \quad 33.79$

33.79

0.00

0.831

$0.7: 30 \quad 792.1370 .000$

SPOEII 1

$570.00 \mathrm{E}$

$570.00 \mathrm{~A} \quad 33.7$

33. 79

$0.00 \quad 695.75 \quad 0.014$

$98: 2.250$ 0.0m

\section{SPLCL 1}

$590.009 \quad 33.79$

33.70

0.270

0.00

מิเ. 75

0.300

$17.312 \quad 9: 3.2000 .0010$

SFGOL. 1

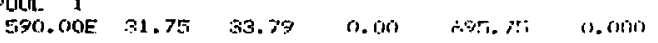

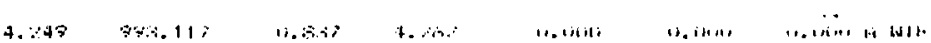

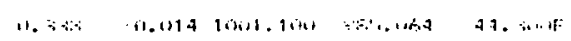

4.29

0.540

4.28

i. insis

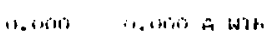

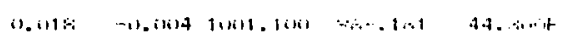

a.ingis

w. niai

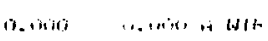

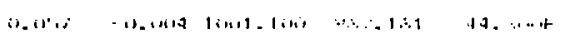

$1.4 \% 5 \% 9311 \%$

$4.3: 30$

$1, \ldots, \cdots \quad$ H.

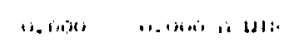
inil.; i. in.t

1). illat:

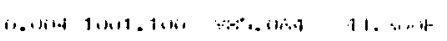
$5 \geqslant 0$

SPOOL 1

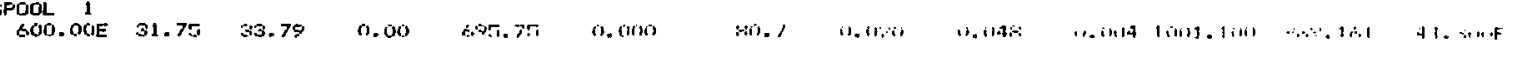

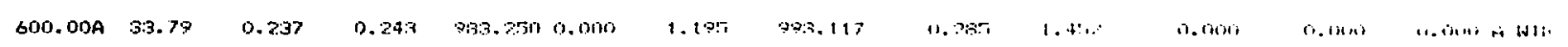

SFCOOL

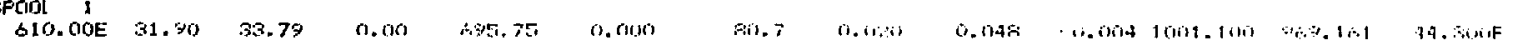

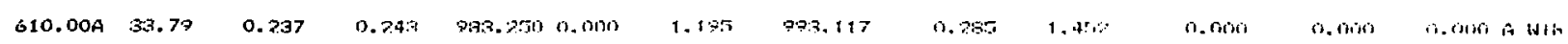

SFOOL 1

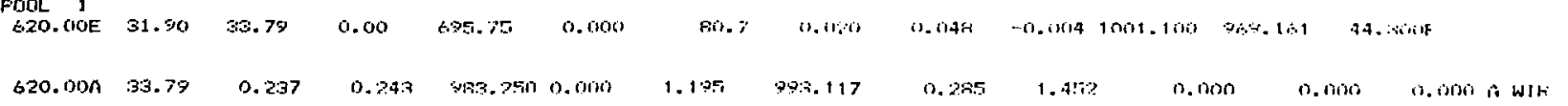

SPOOL 1

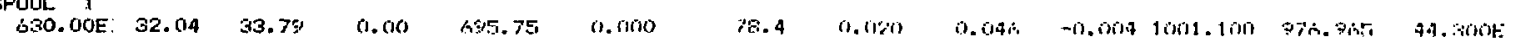

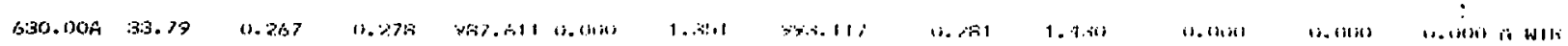




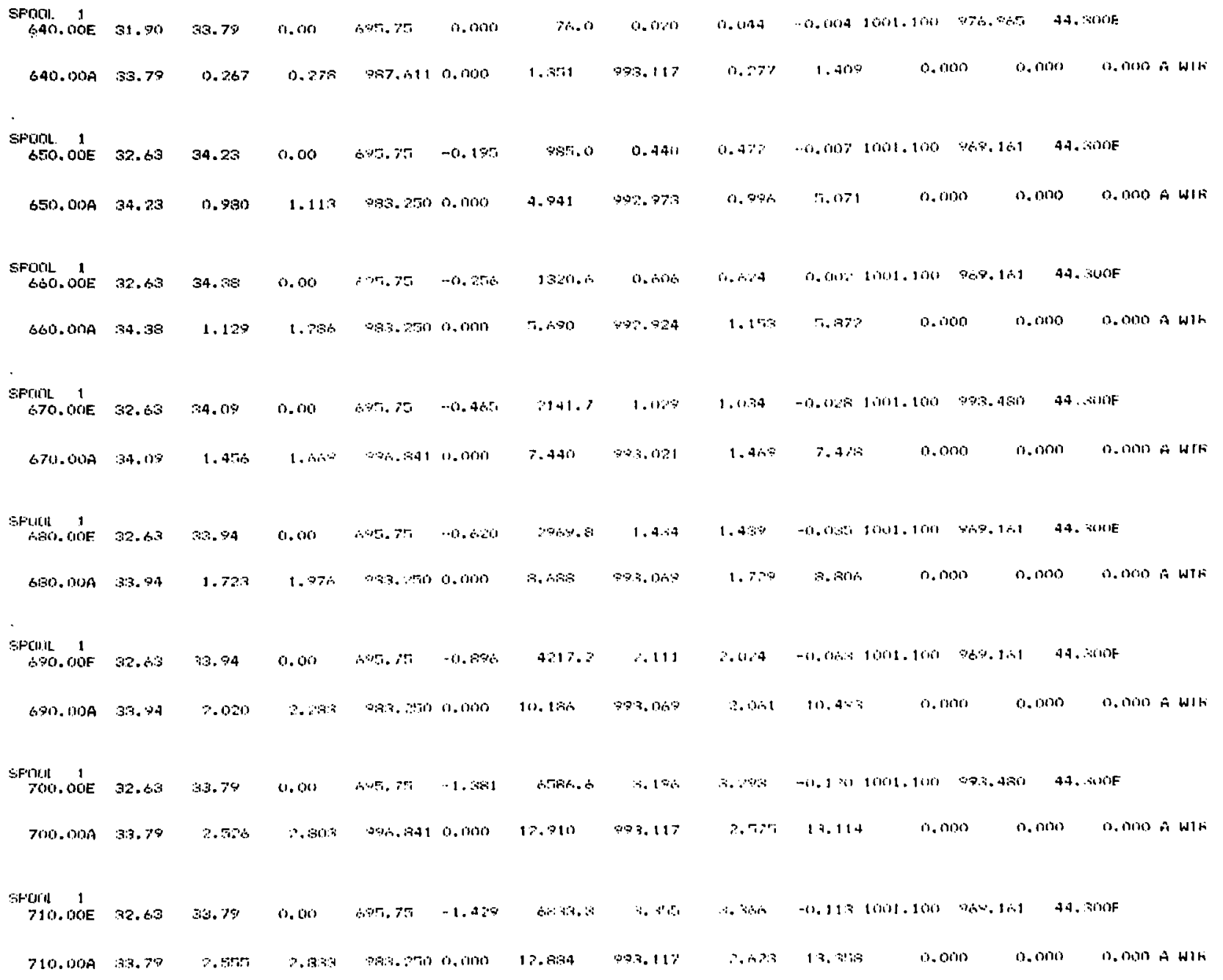




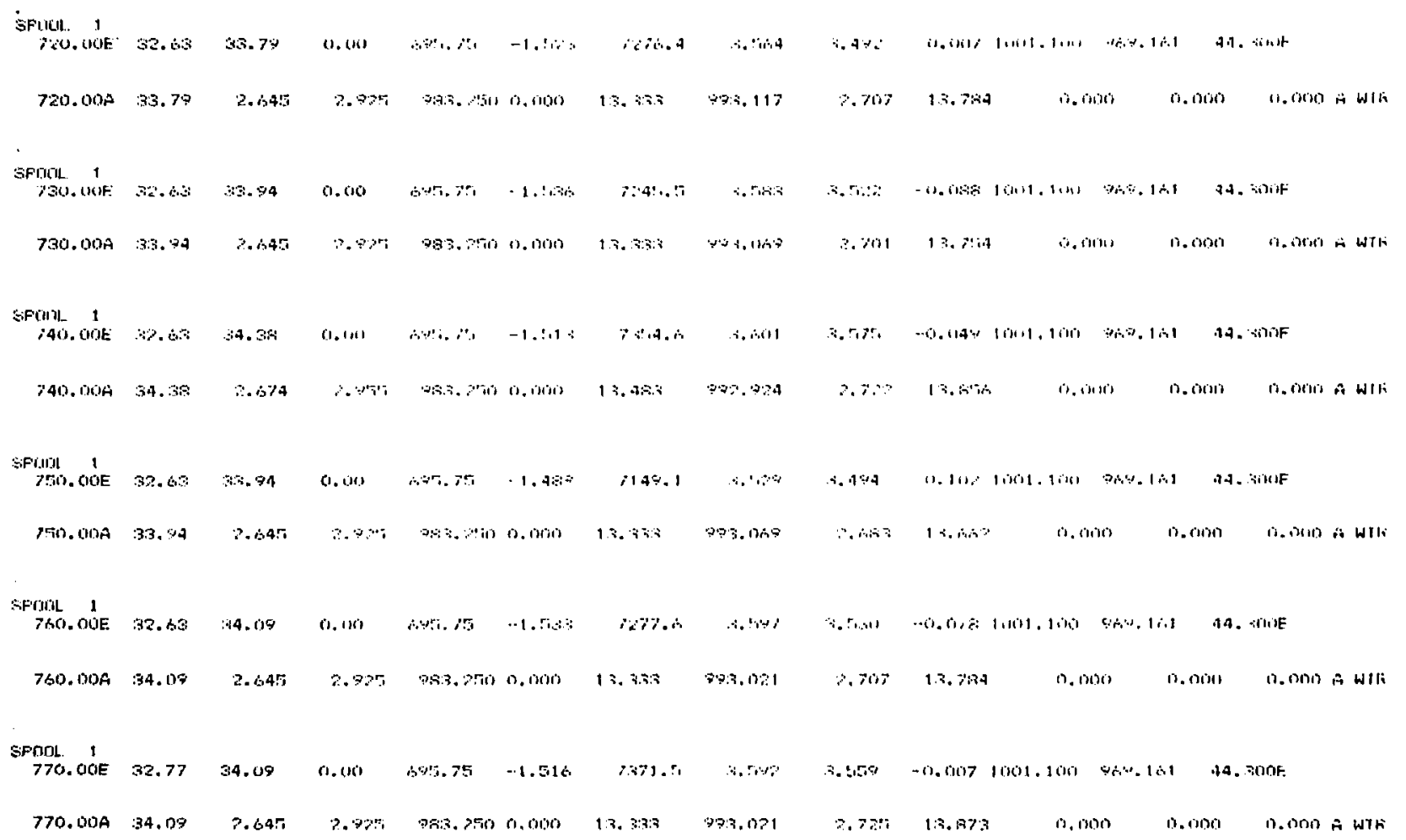




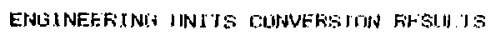

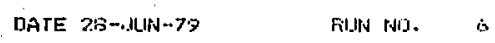

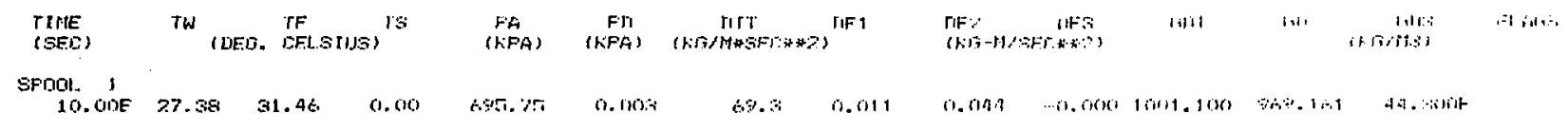

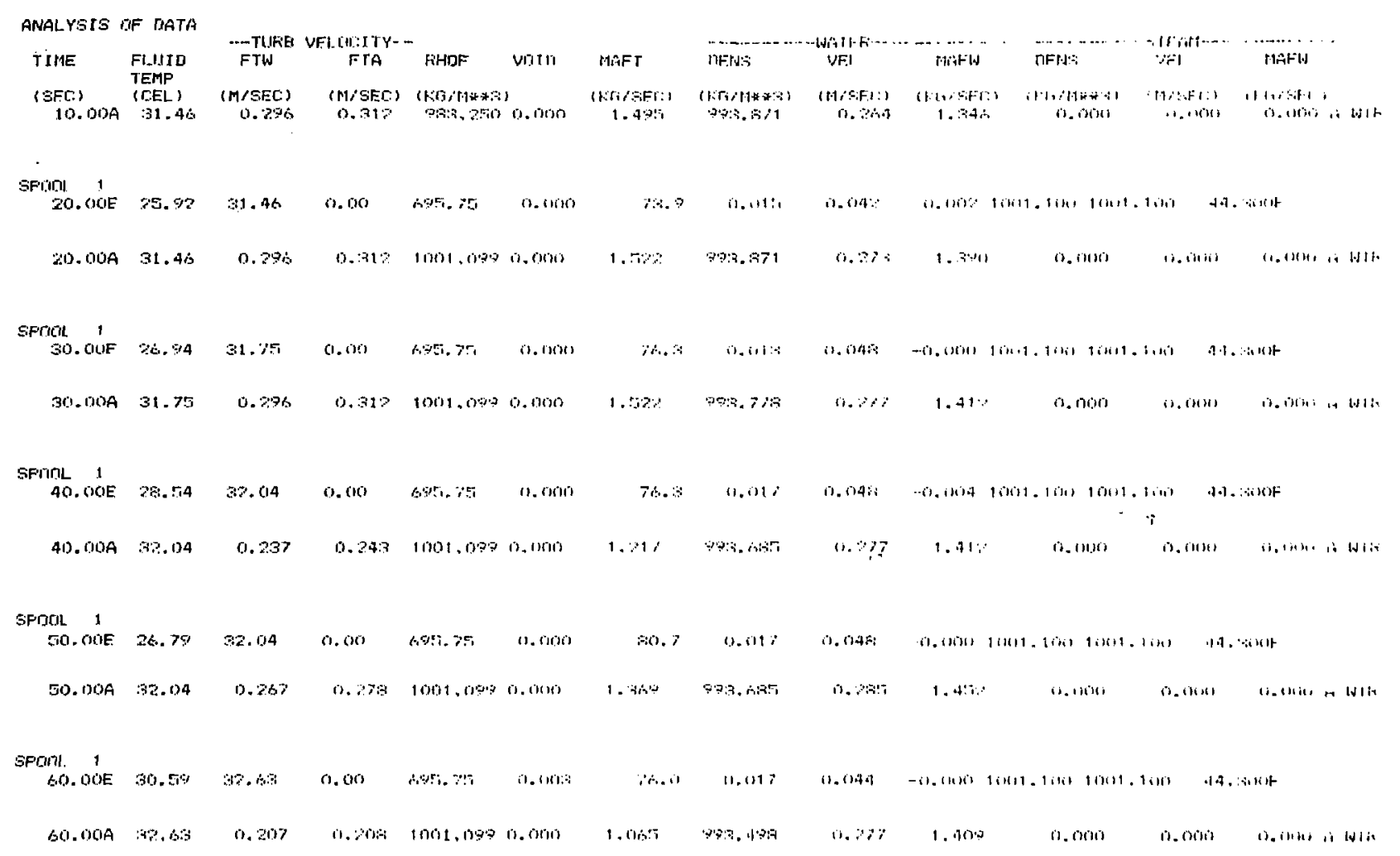




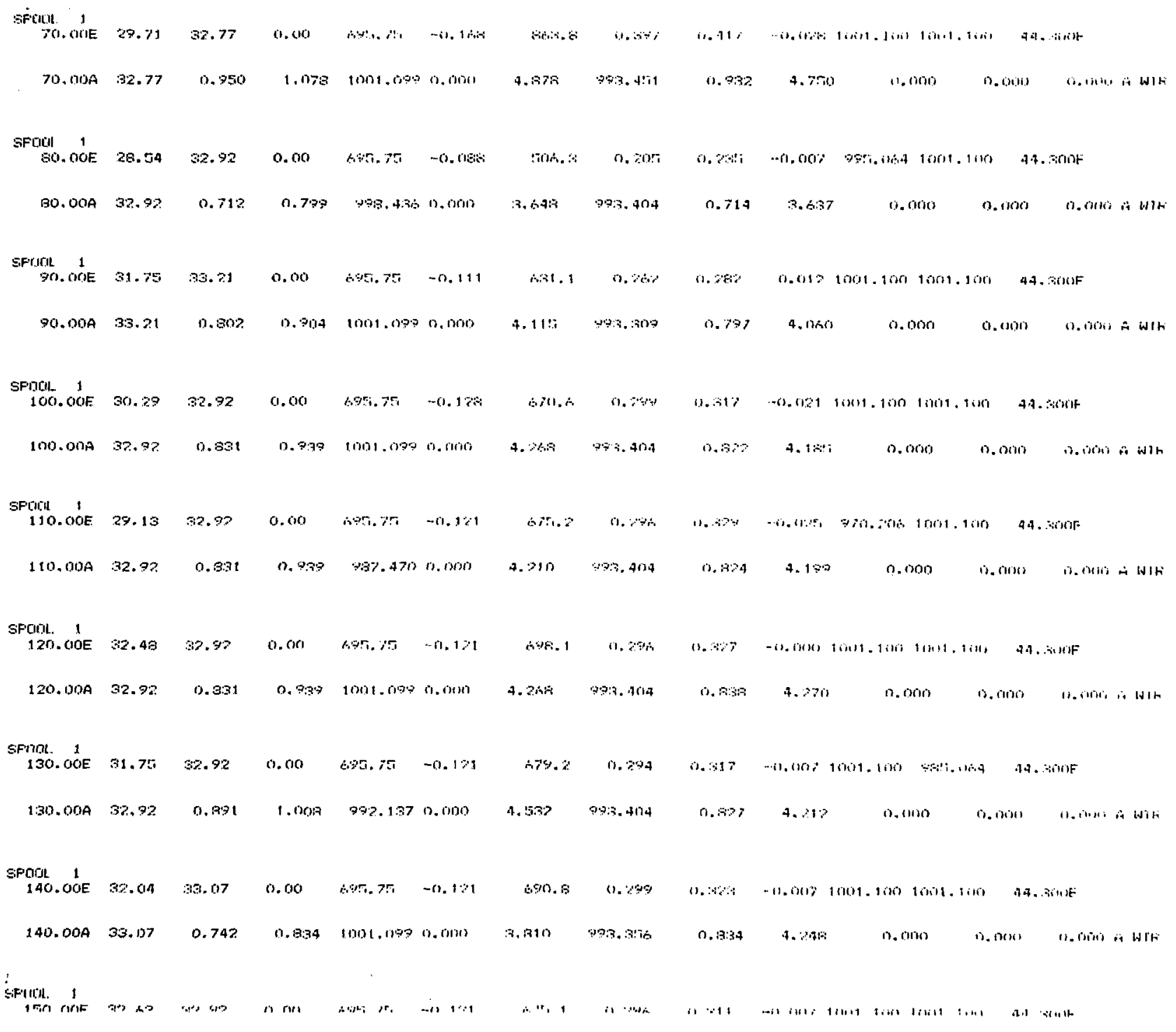




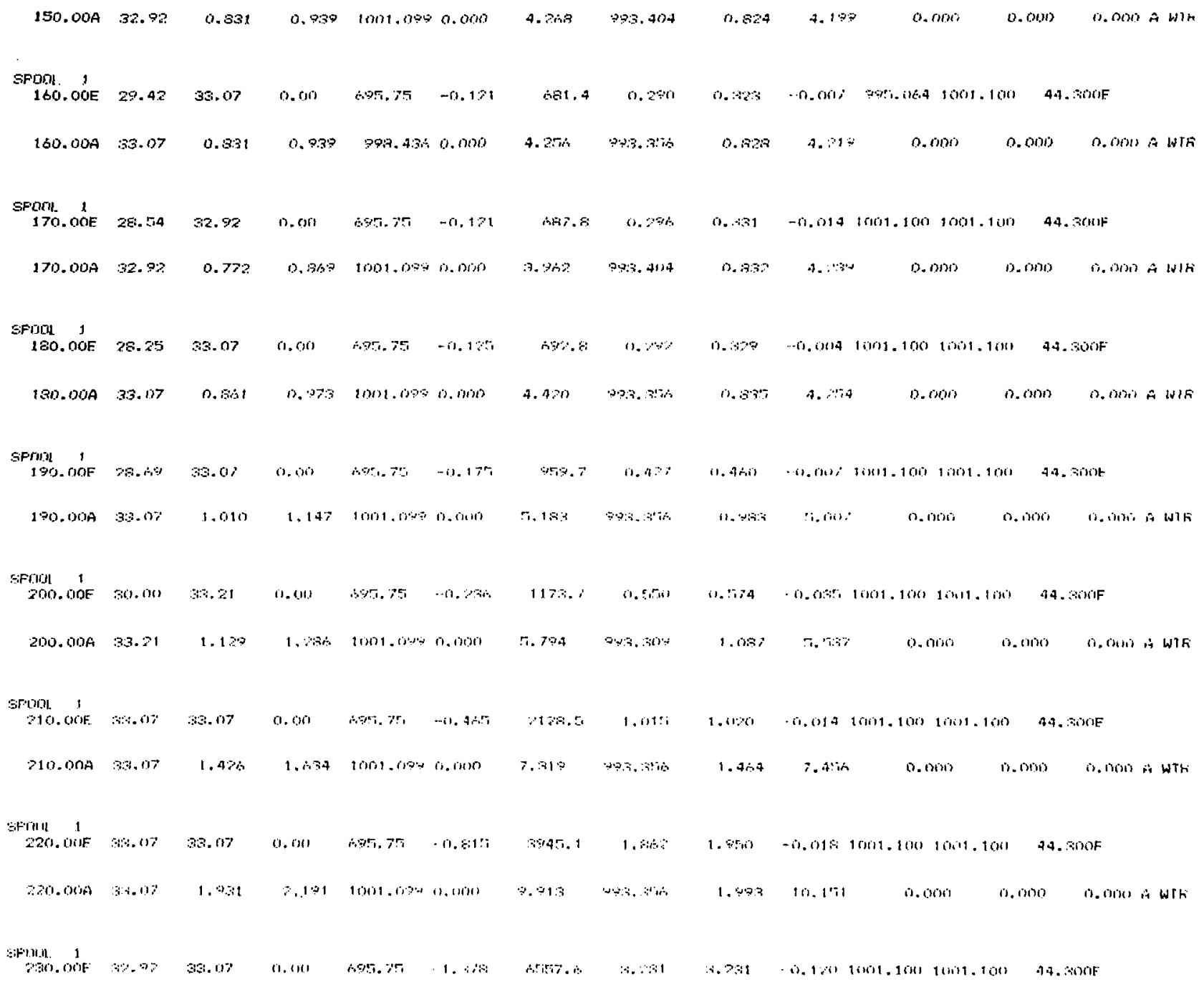




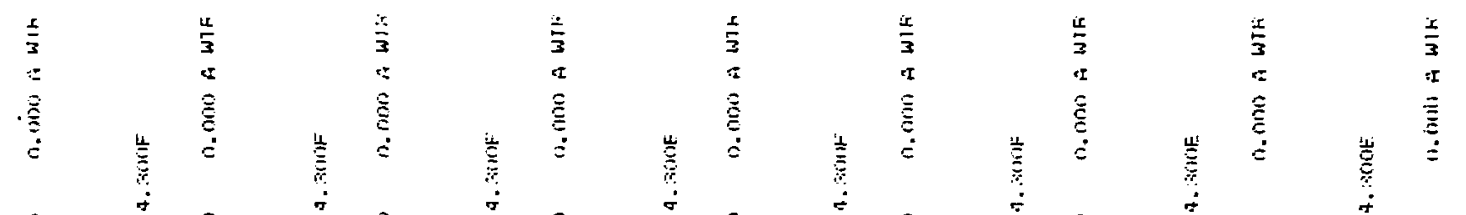

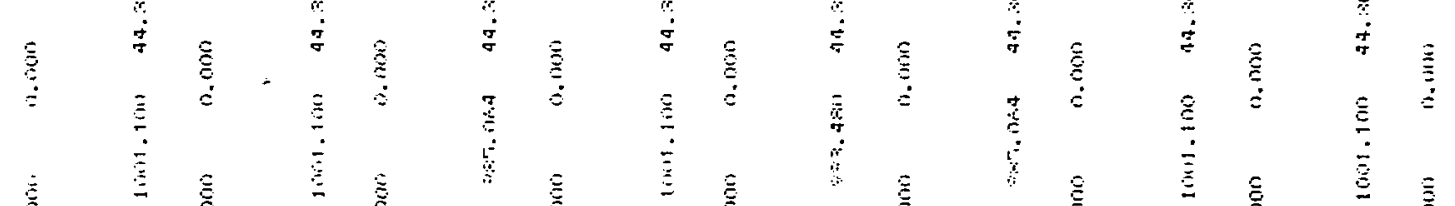

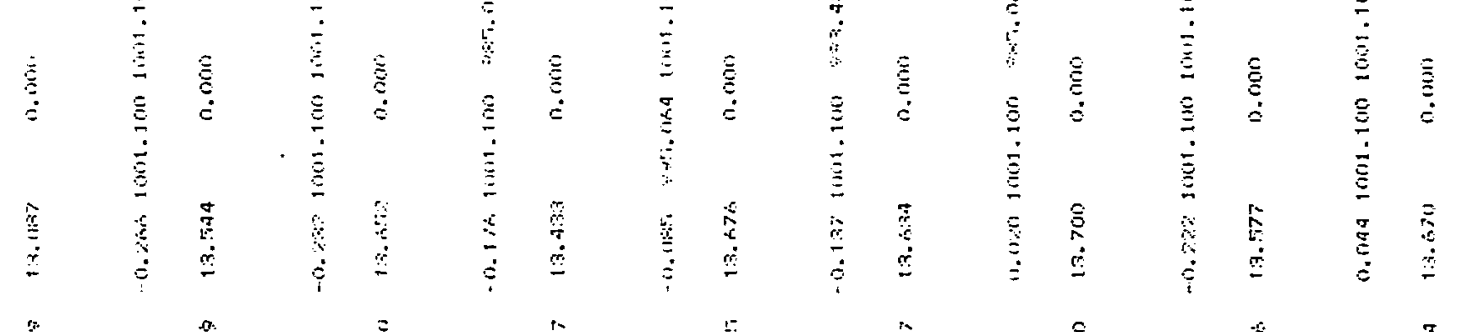

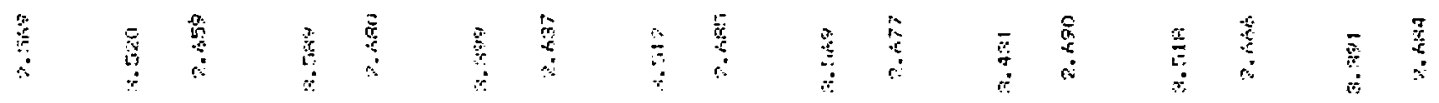

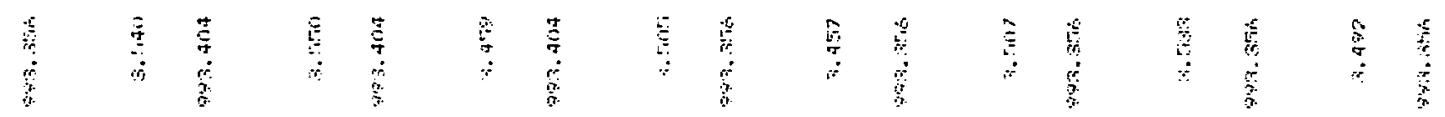

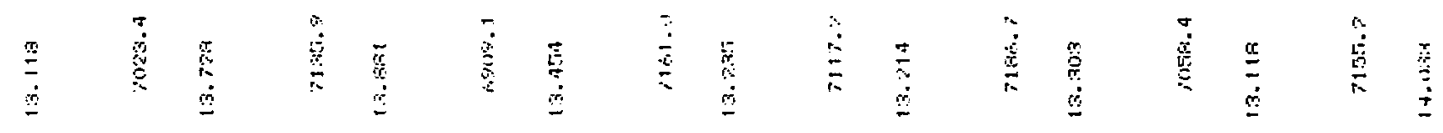

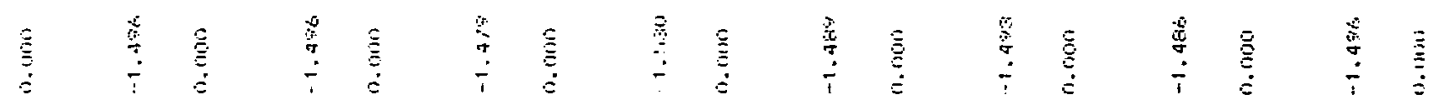

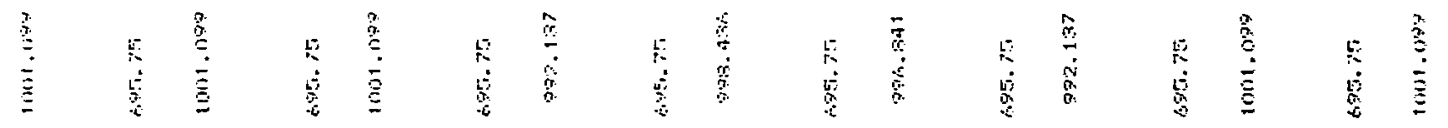

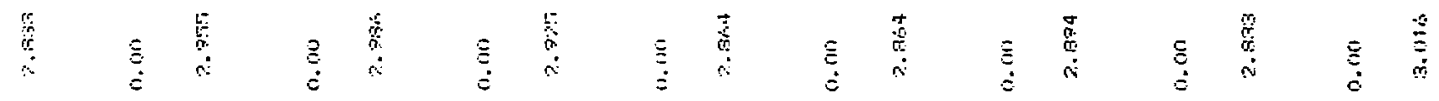

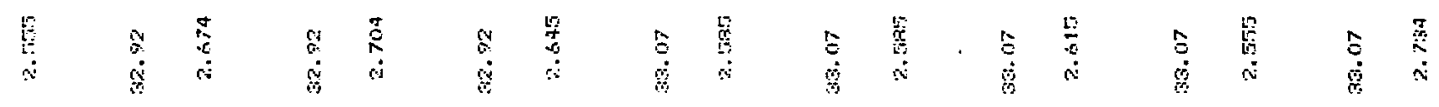

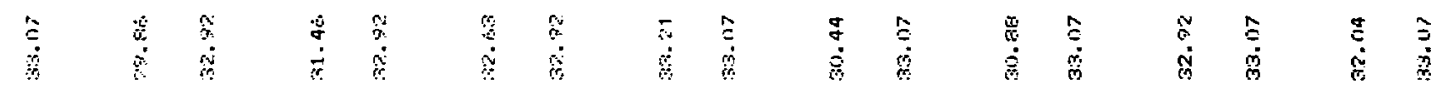

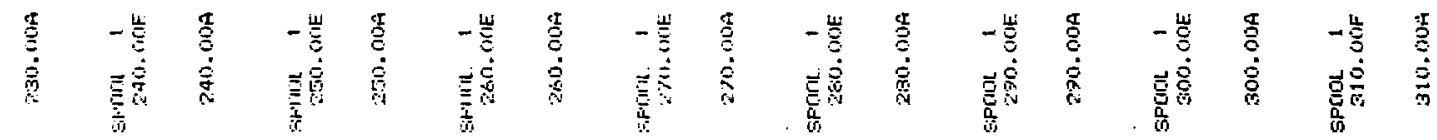




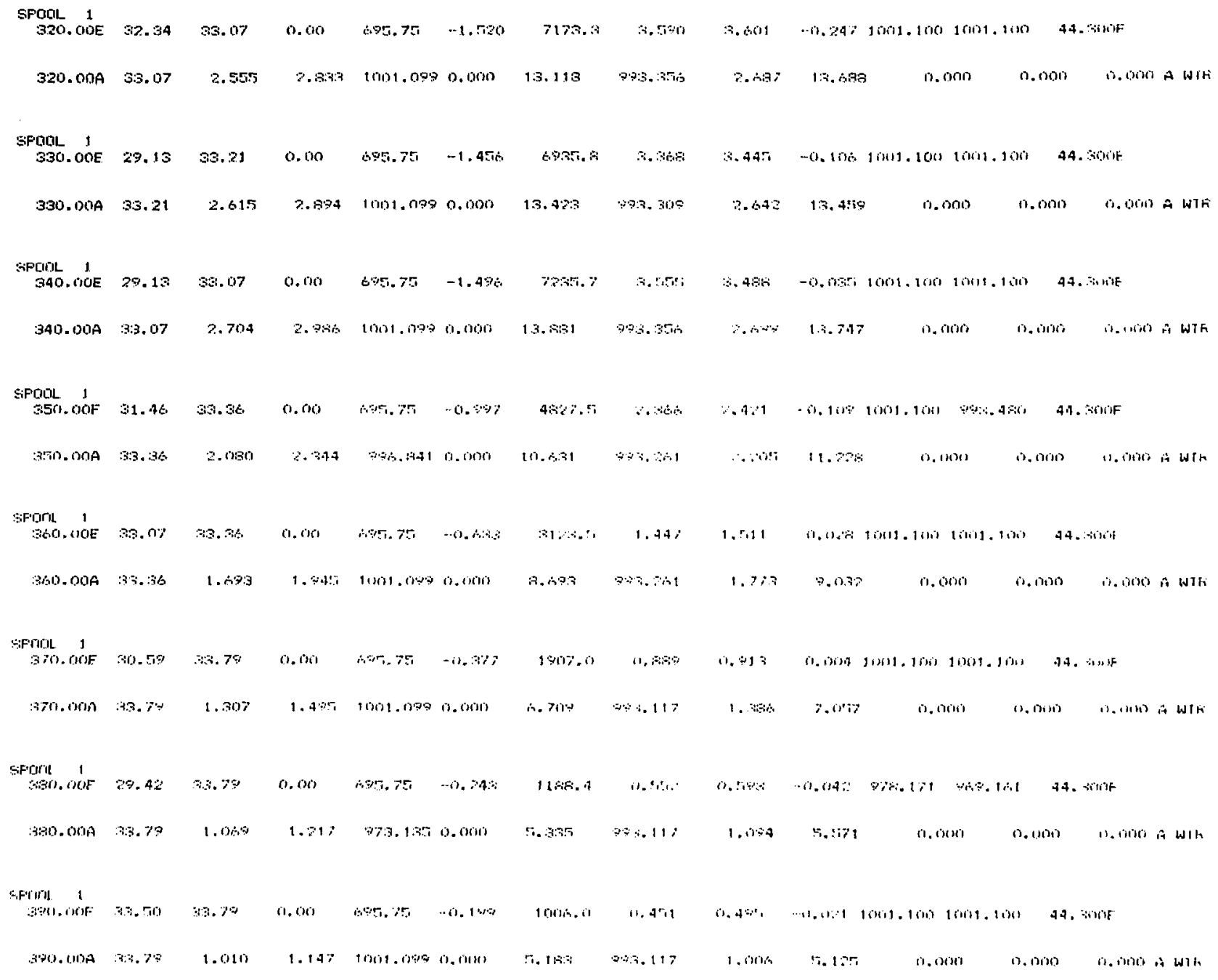




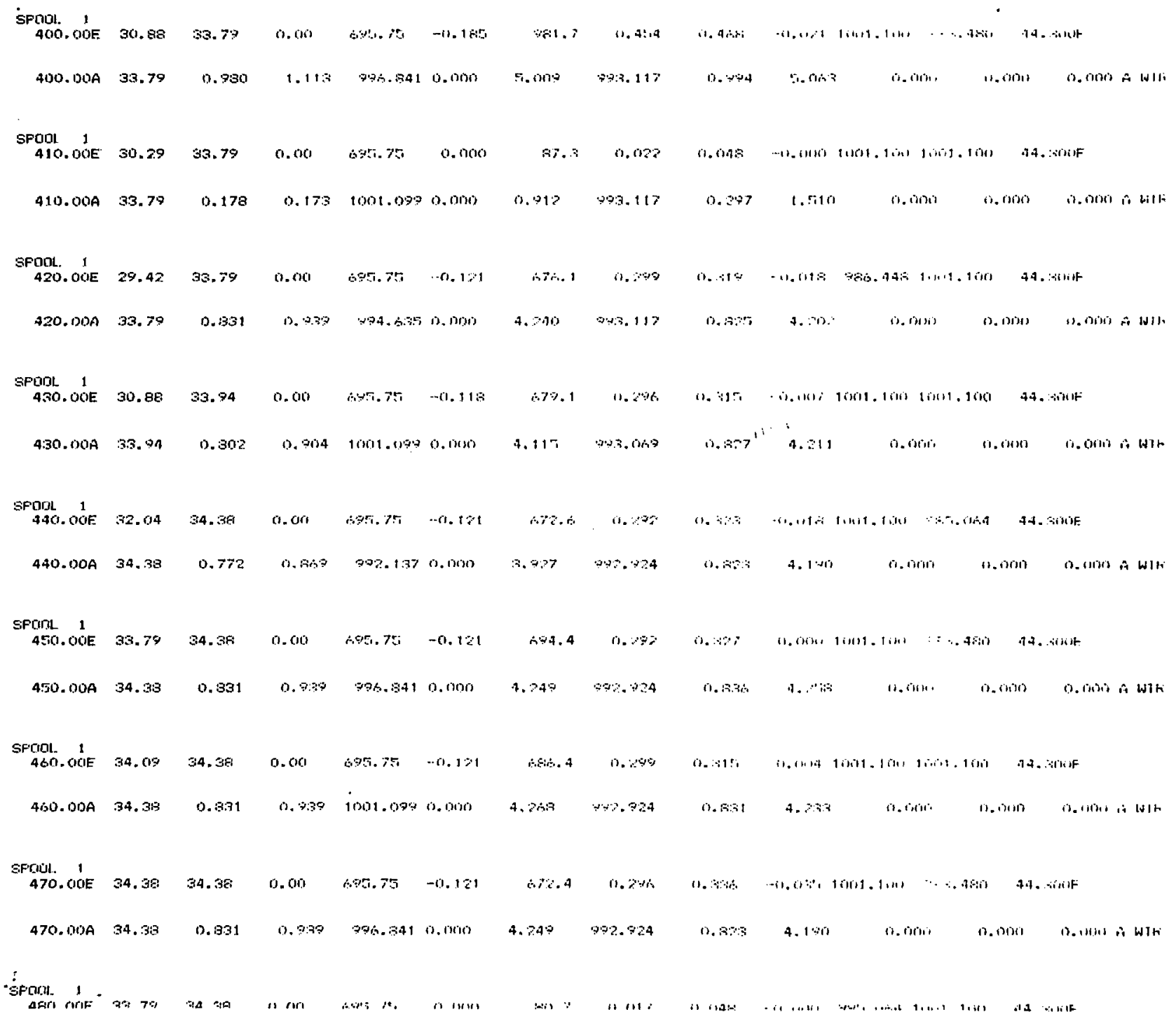




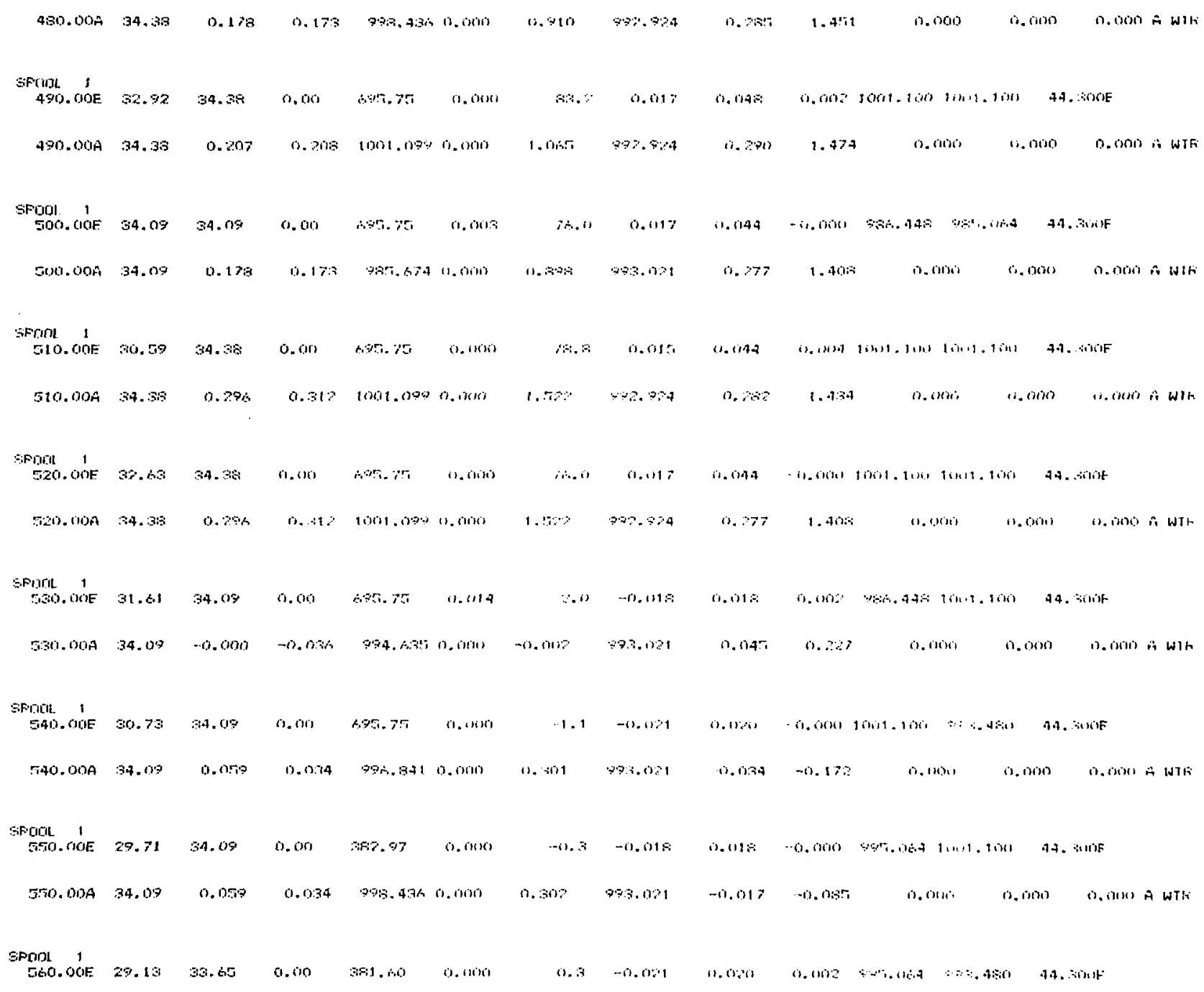




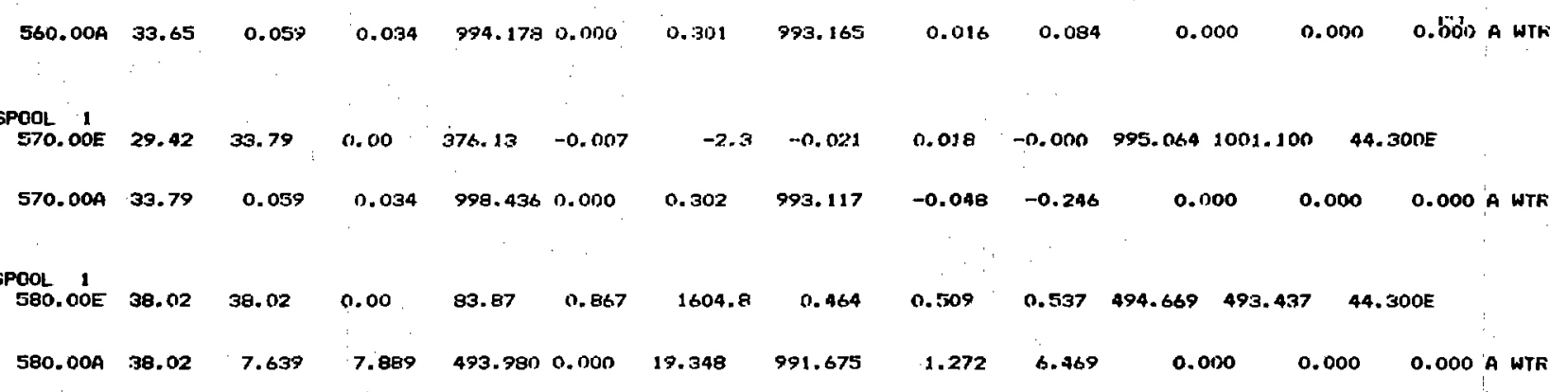




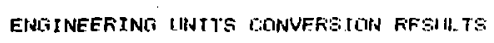

DATE $23-111 N-79$ RIINS NIT, $;$

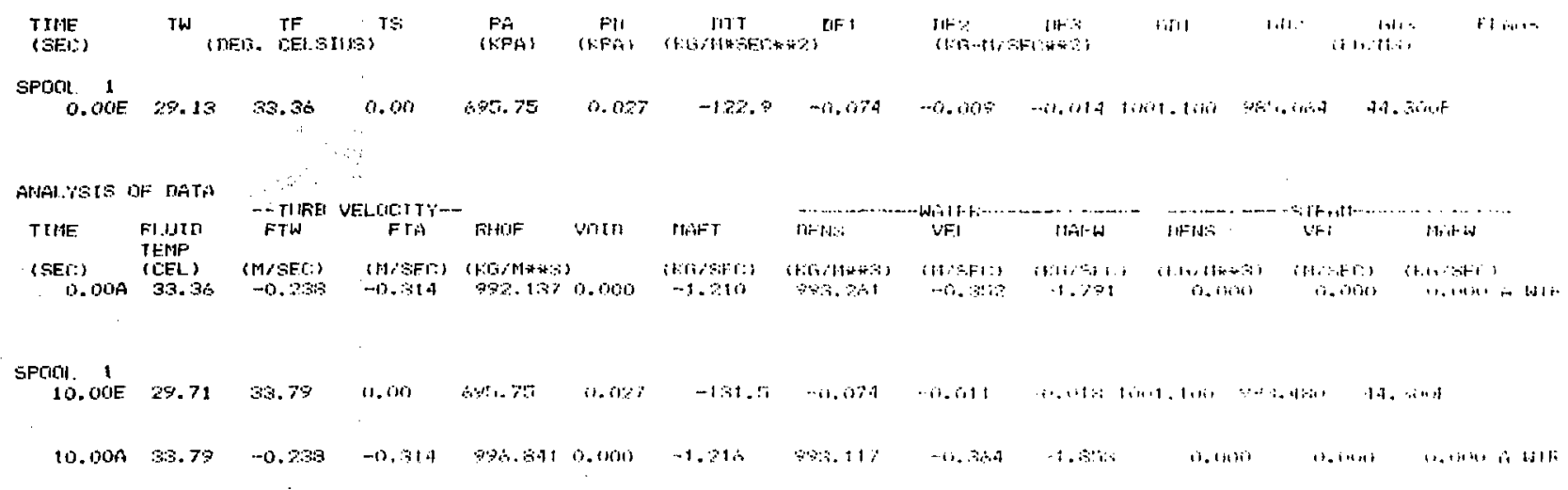

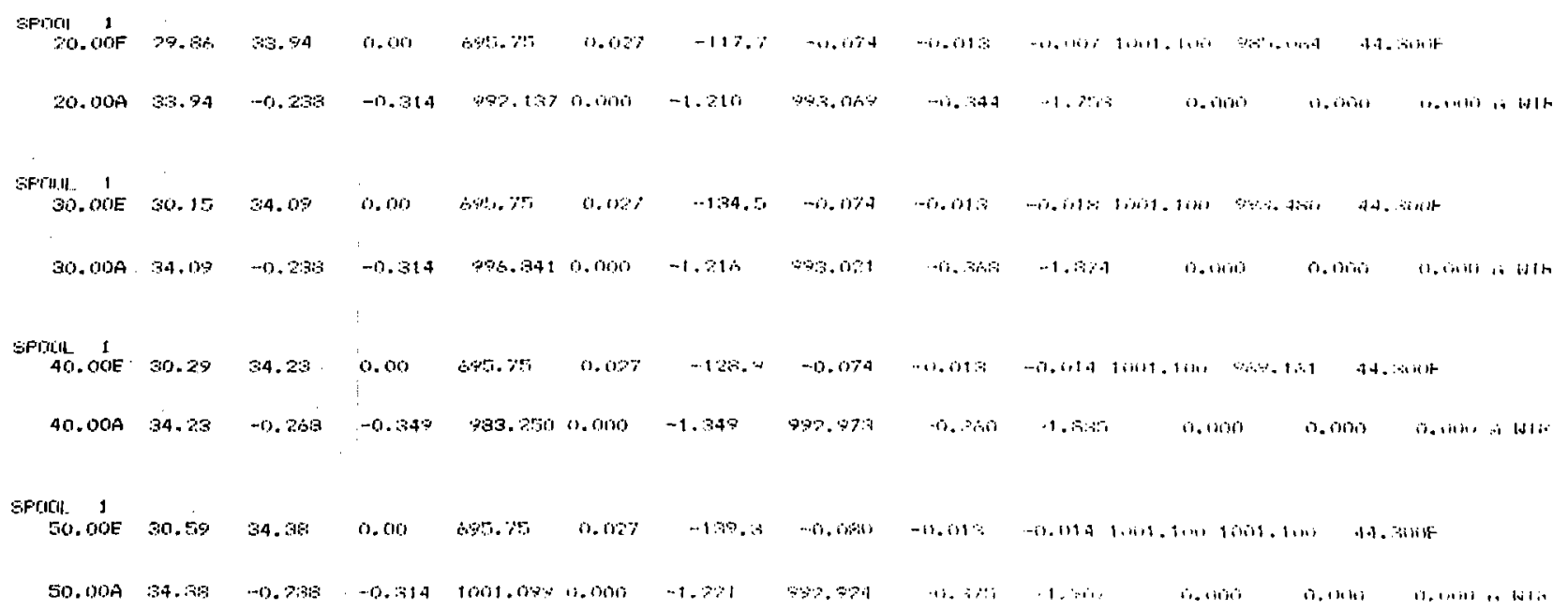




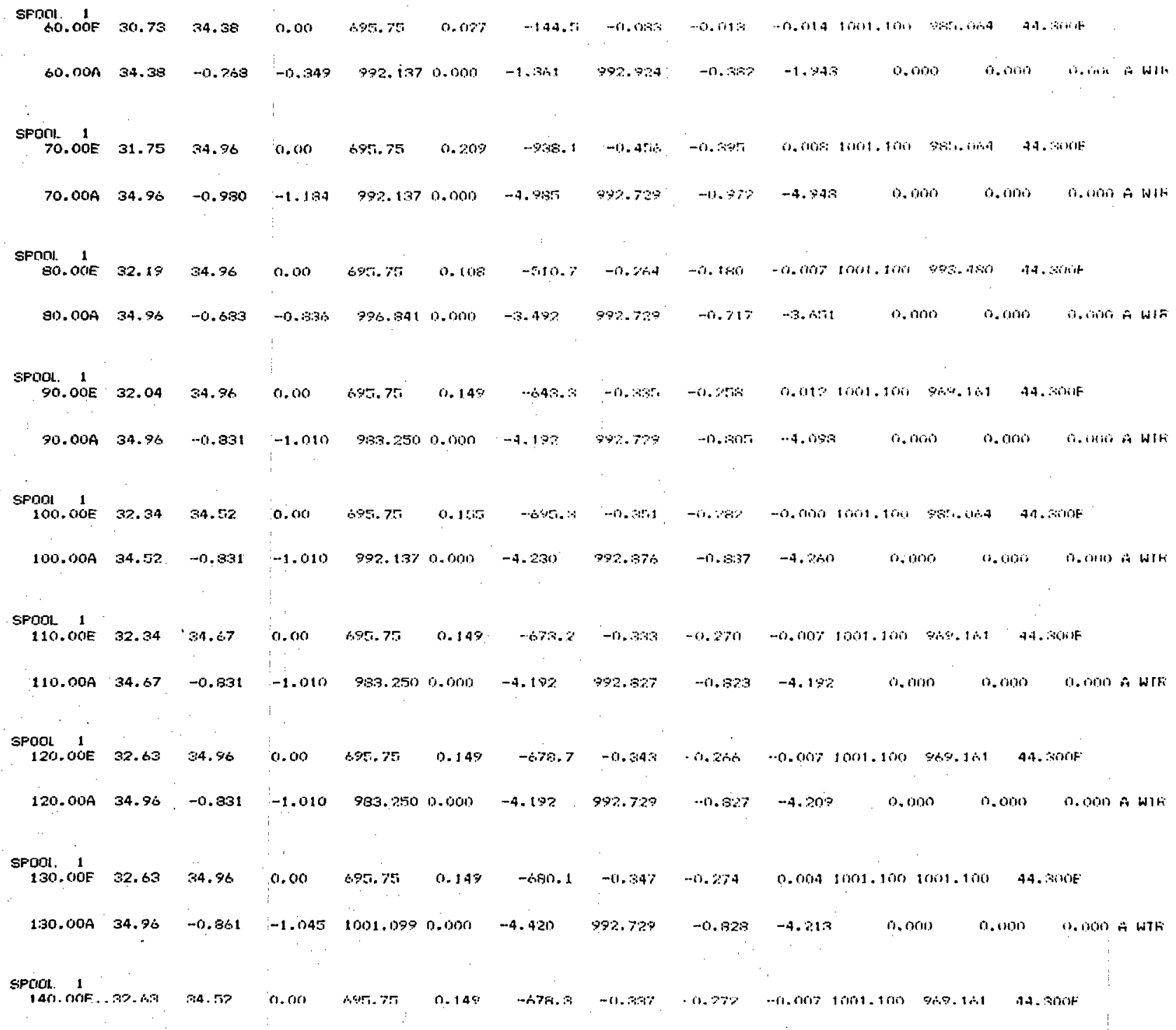




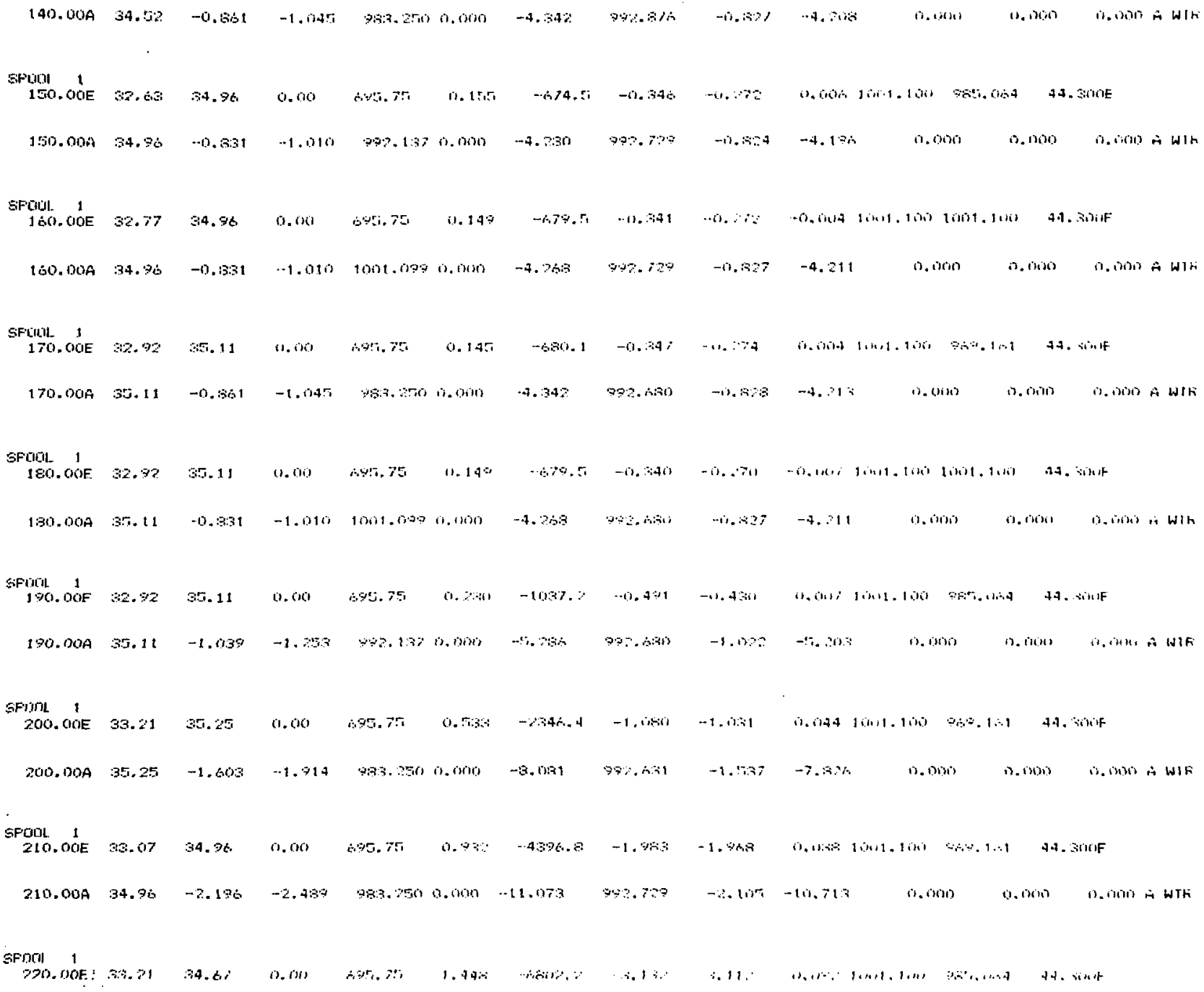




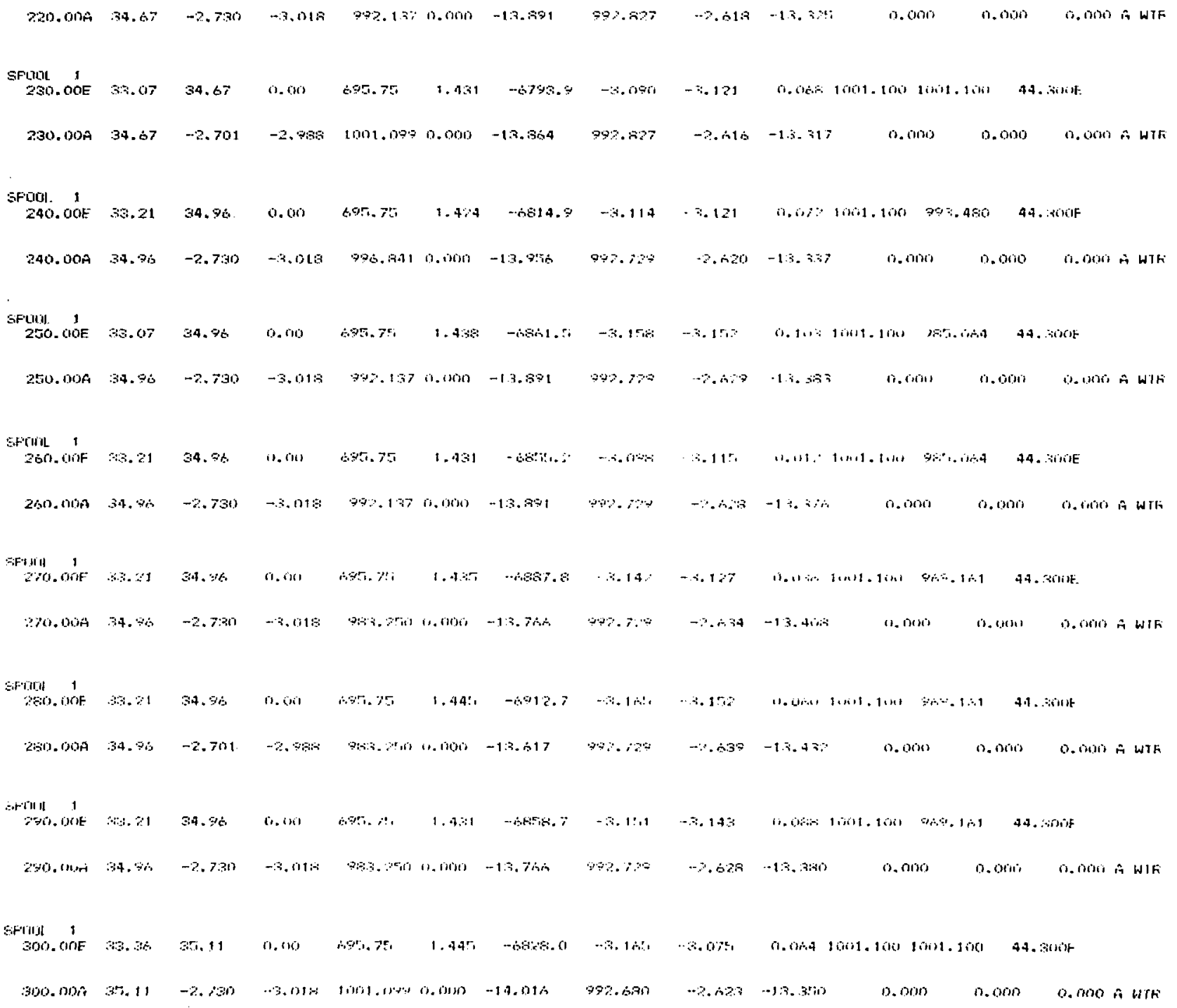




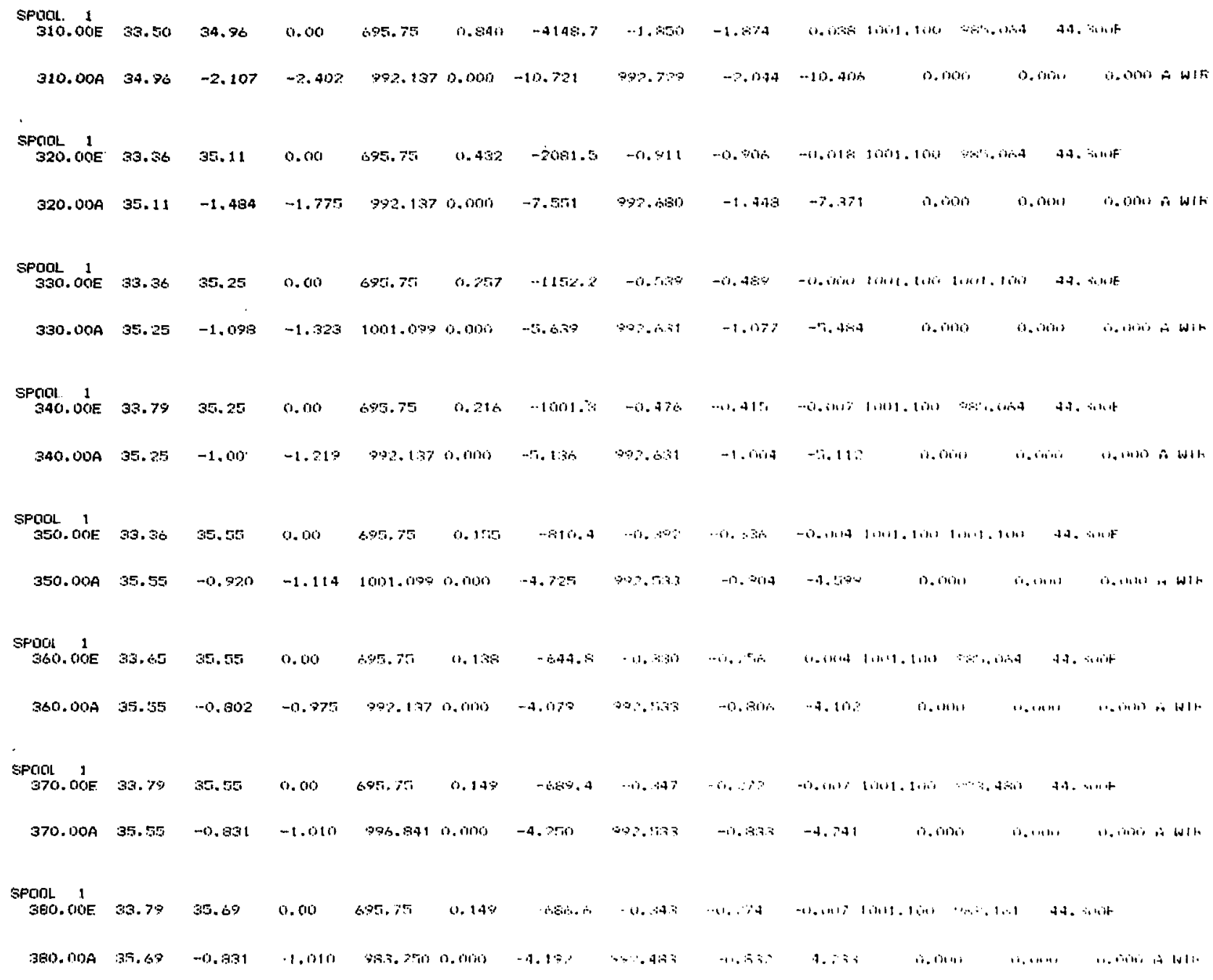




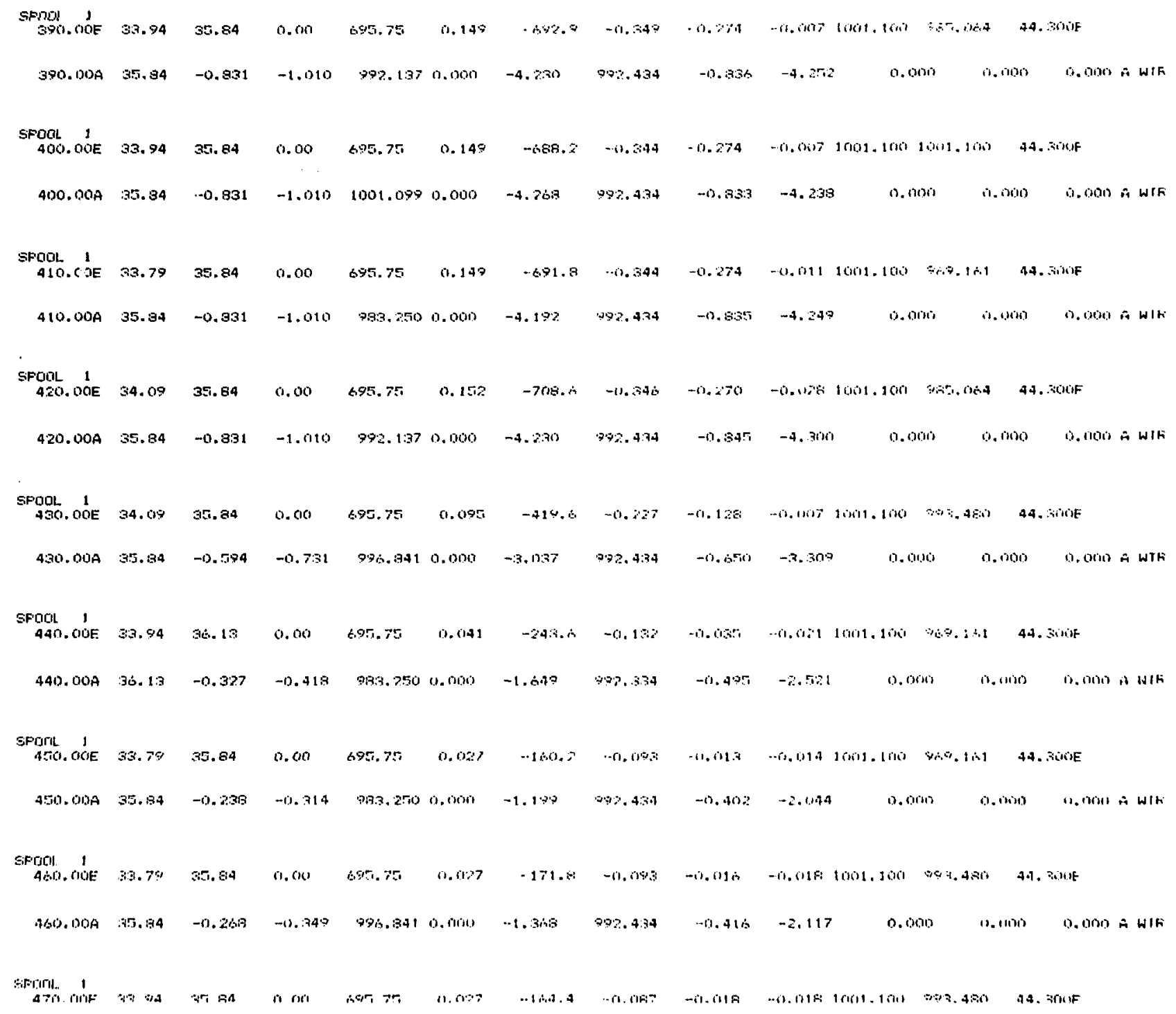




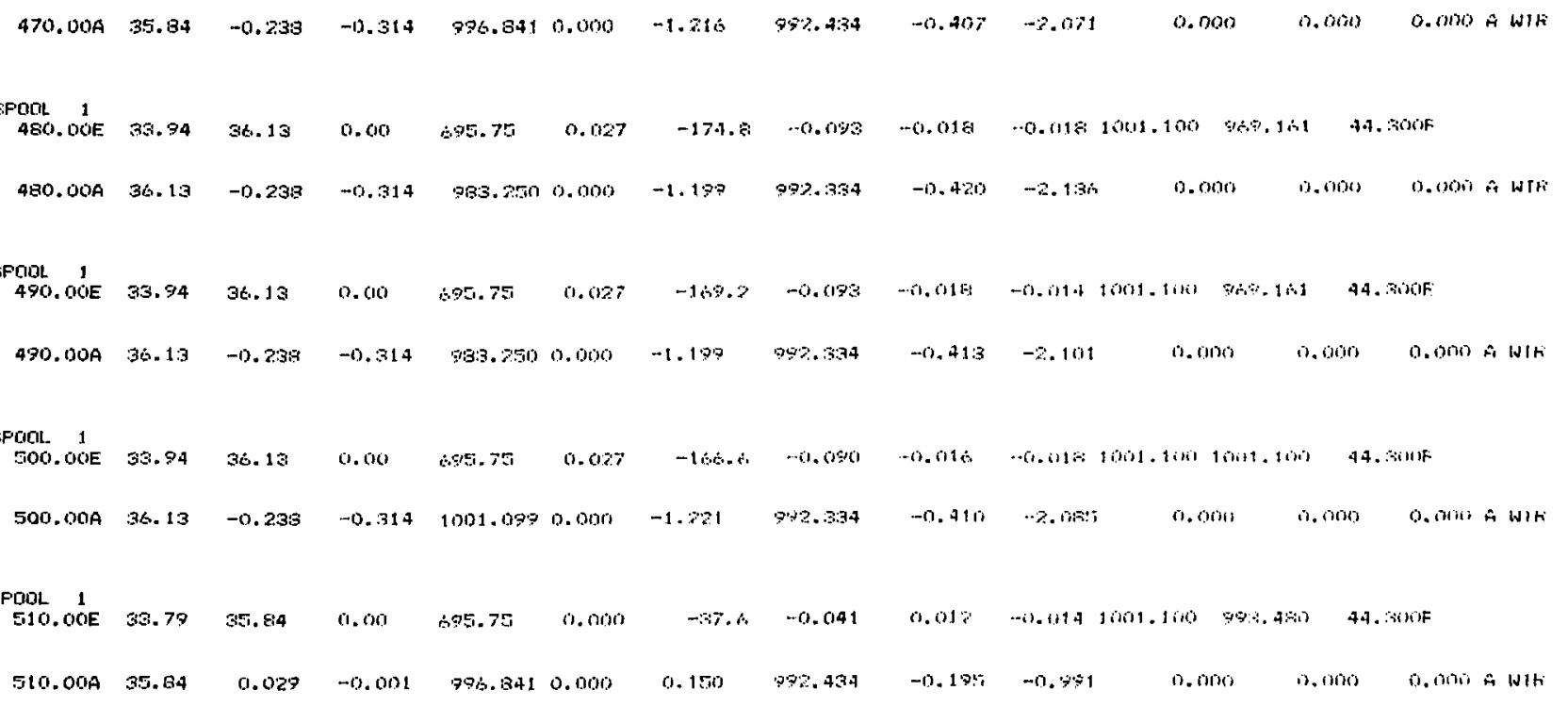


EINTIINFERINII IINTIS CONVERSINAN RESIIITS

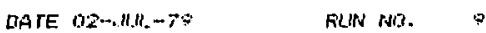

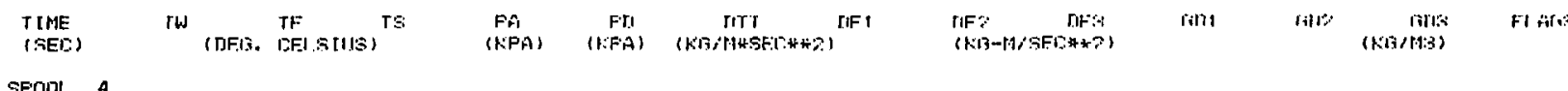

SFTIIII. 4

10. $\mathrm{nOF} \quad 24.45 \quad 26.79$

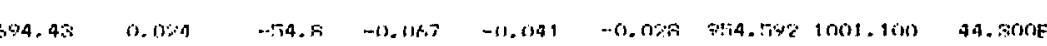

ANAI.YSIS DF MATA

\begin{tabular}{|c|c|c|c|c|c|c|c|c|c|c|c|c|}
\hline & & & Ut:1 Itr:1 1 Y- & & & & & 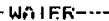 & $\cdots$ & & $-S T$ & 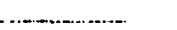 \\
\hline TIME. & $\begin{array}{l}\text { FI.IIID } \\
\text { TFFIF }\end{array}$ & FTW & Fra & RHIOF & לוד & MAFT & DENS & VET. & MAFW & DFRS & VE.I. & MAFW \\
\hline (SEC:) & (CEE) & (M/SEC) & (MISEC) & 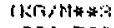 & & (1:THISEC: & kni & Miseril & thesi & 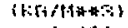 & $\{M / S i F r)$ & $z(x)$ \\
\hline $10.00 \mathrm{~A}$ & $2 A .79$ & -0.136 & -17.120 & 780.58 & 0.000 & $-0,4 B b$ & 995,2813 & -10.235 & $-1.19 B$ & 10.000 & 0.000 & $0.000 \mathrm{~A}$ \\
\hline
\end{tabular}

sFina.

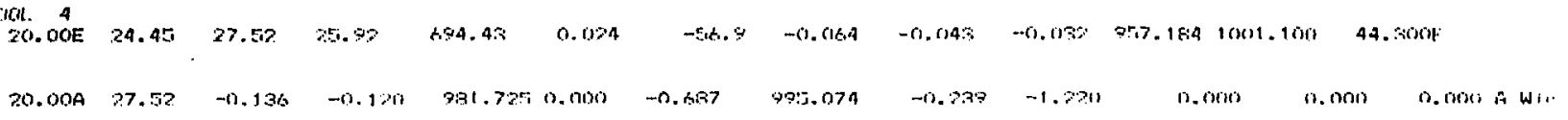

ต

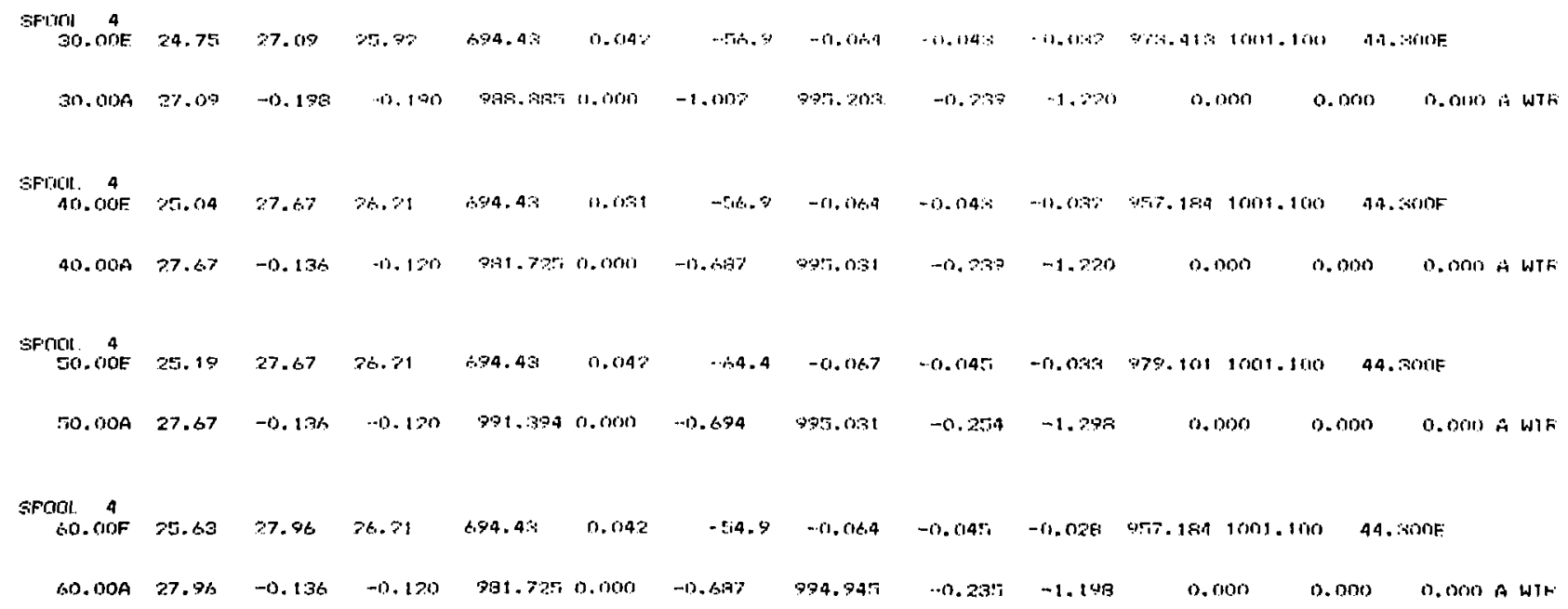




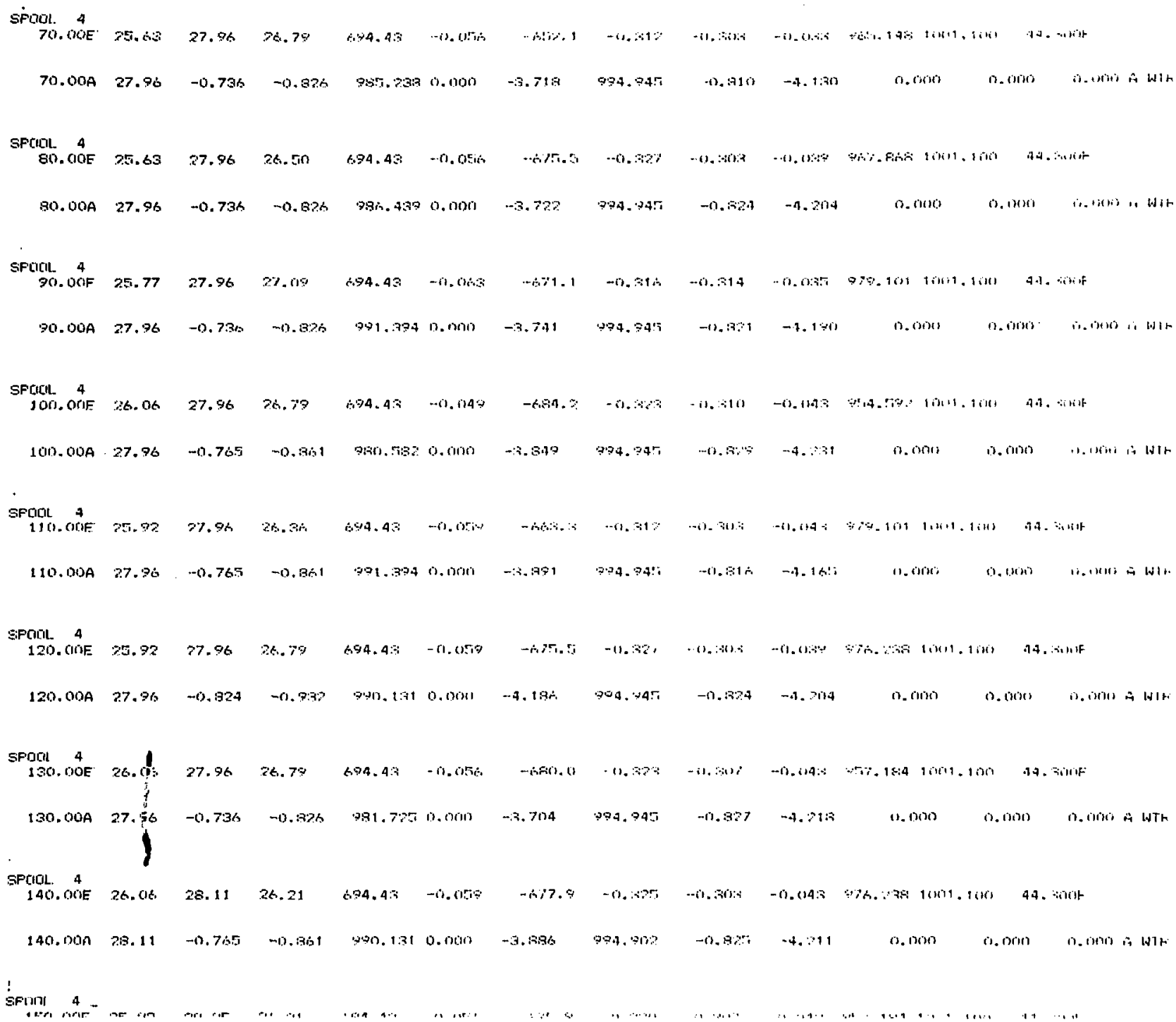




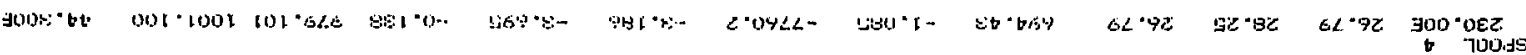

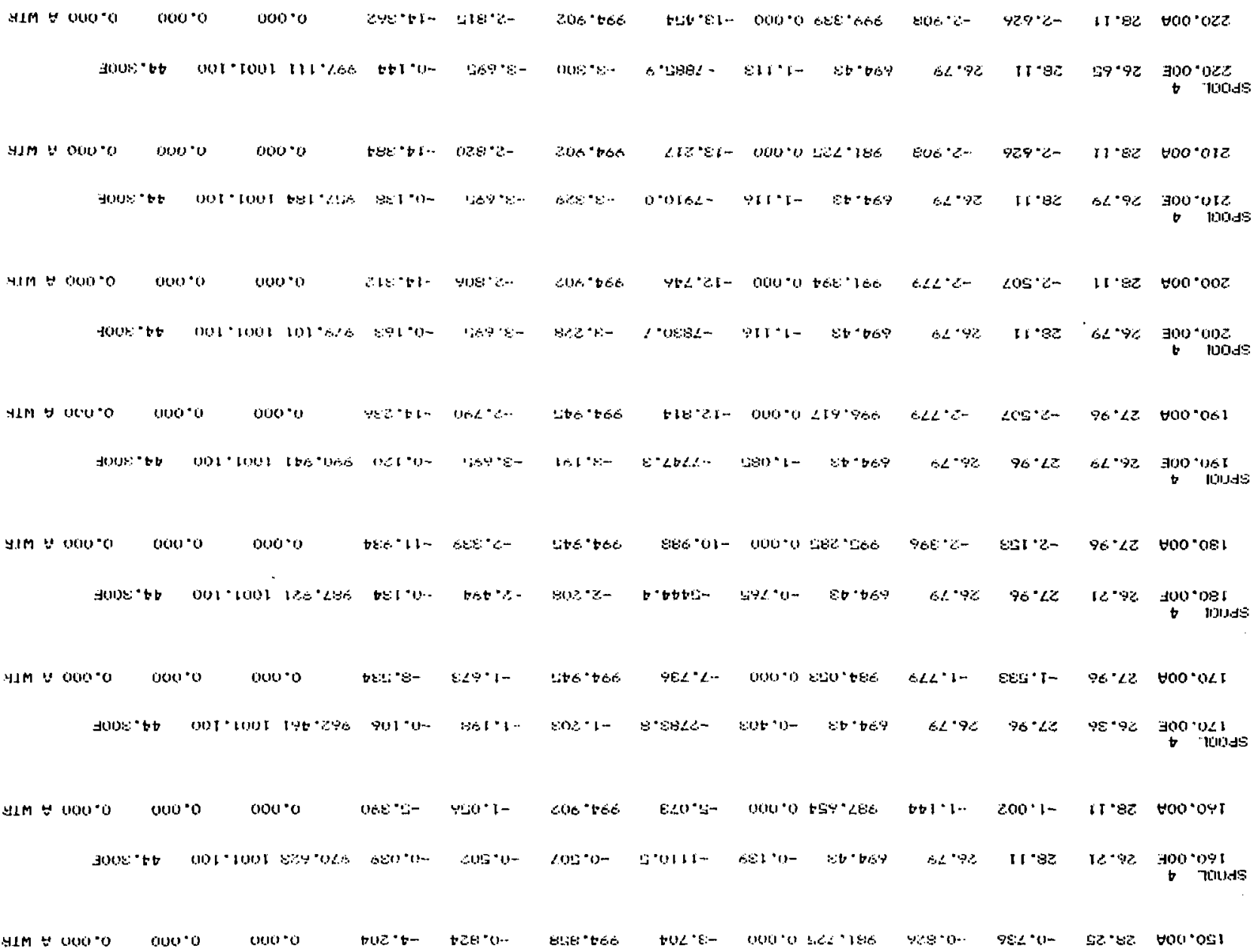

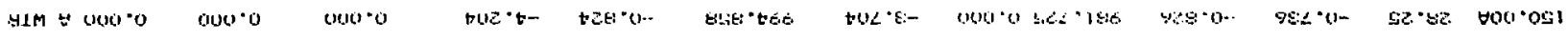




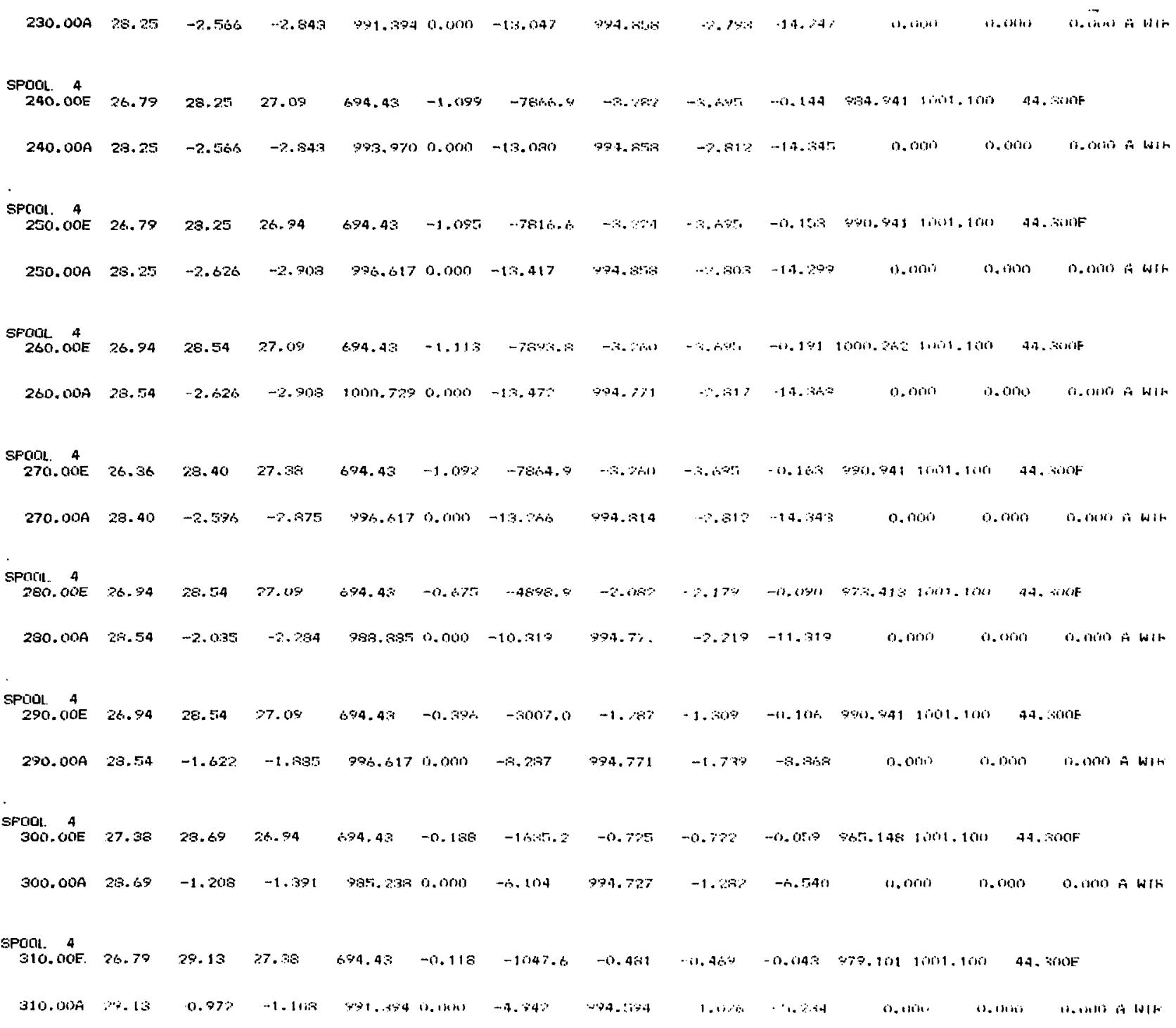




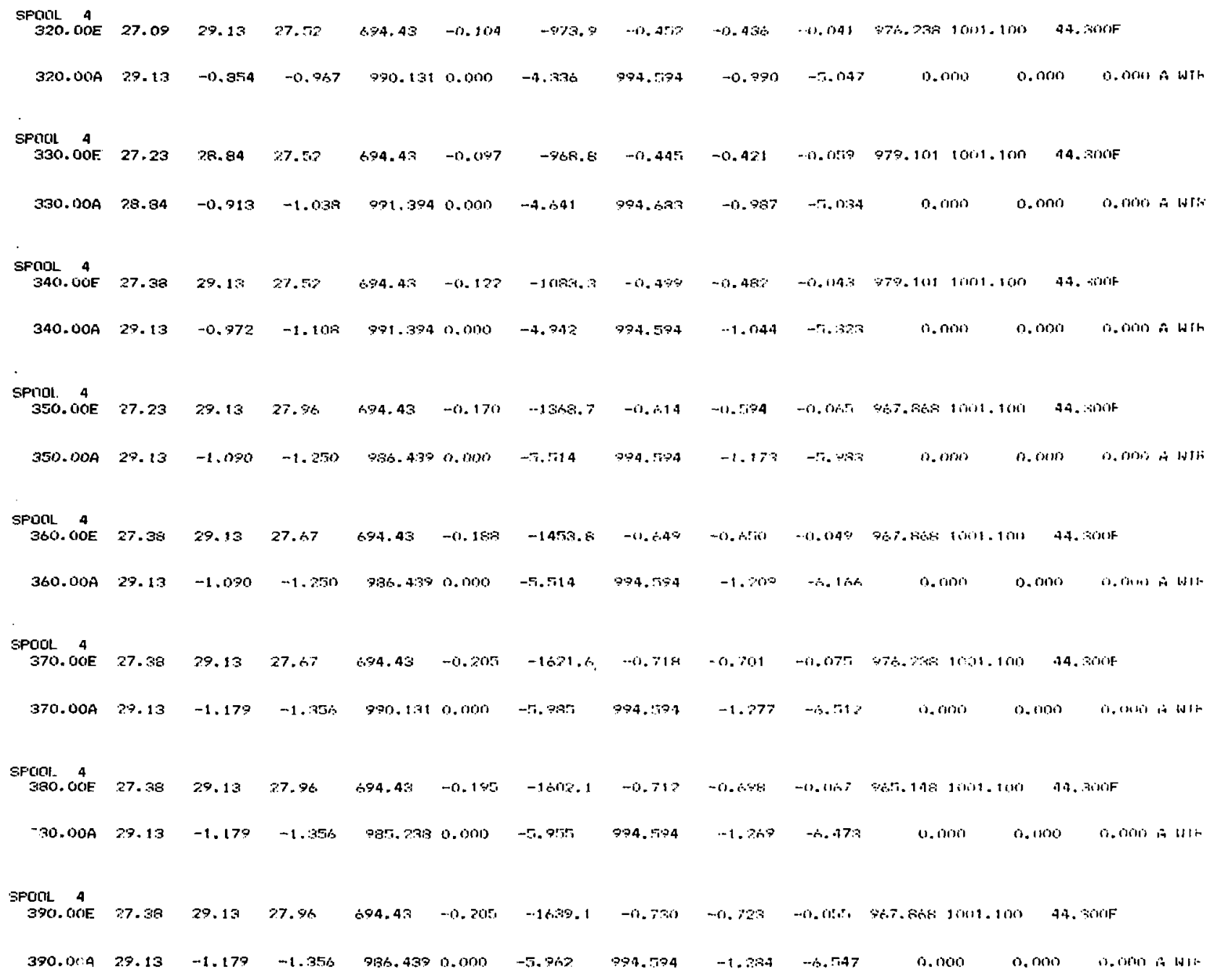




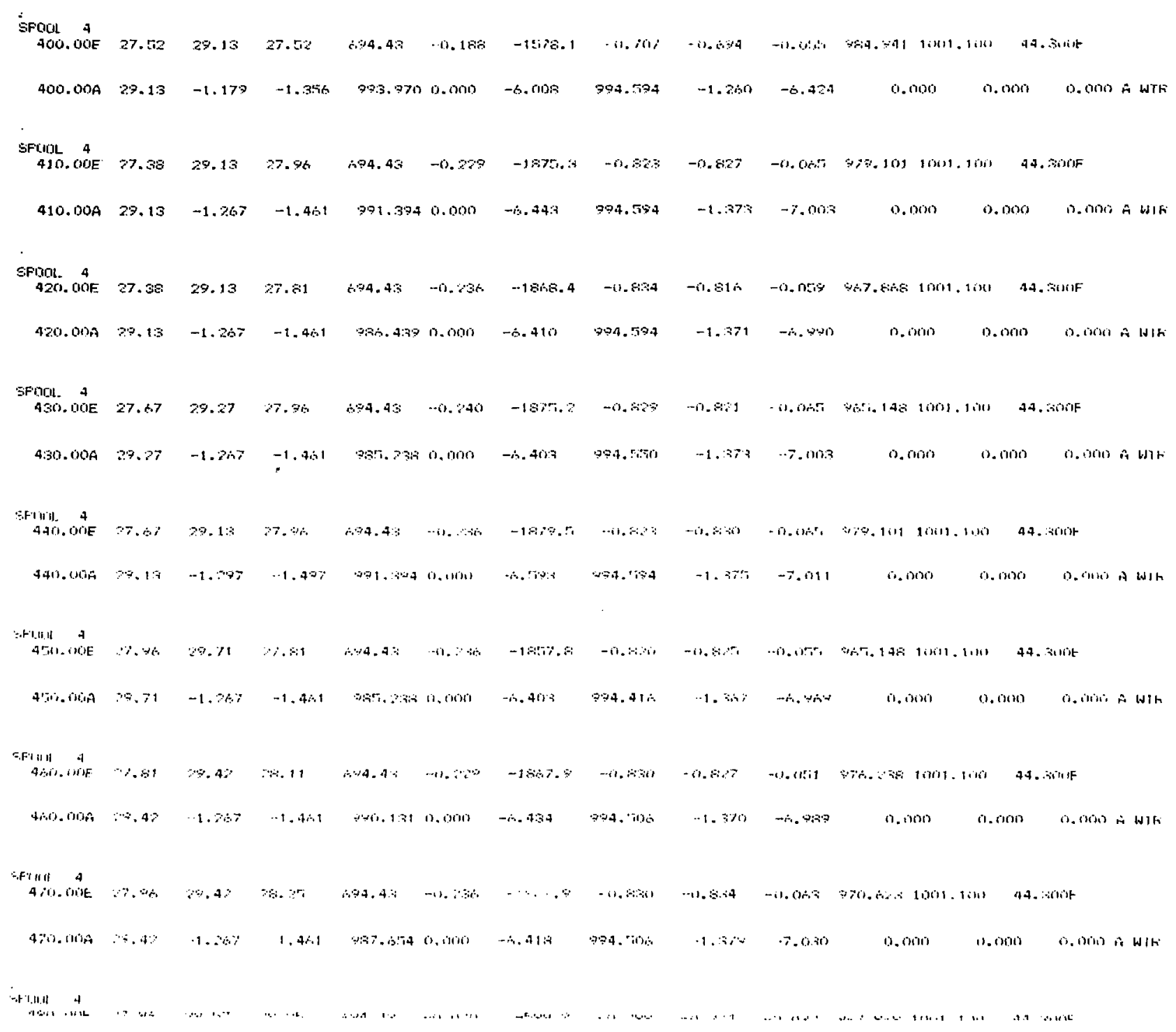




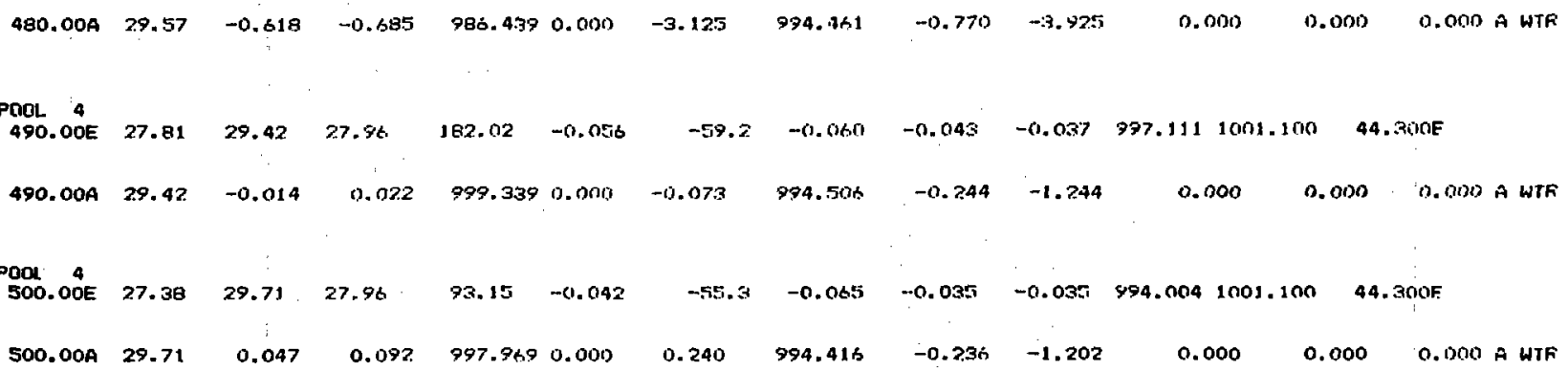


ENOINEERING UNITS CONVERSION RESIIITS

DATE O2-JUH.-79 RIIN NIT. 10

\begin{tabular}{|c|c|c|c|c|c|c|c|c|c|c|c|c|}
\hline $\begin{array}{l}\text { TIME } \\
\text { (SEC) }\end{array}$ & TW & IDEGS. & $\begin{array}{l}\text { TF } \\
\text { CELISIISS) }\end{array}$ & TS & $\begin{array}{l}F A \\
\left(K^{\prime} P A\right)\end{array}$ & $\begin{array}{c}\mathrm{FI} \\
(\mathrm{FA})\end{array}$ & $\underset{\left.\left(K 13 / P+S F_{1}\right)+2+2\right)^{\mathrm{DF} 1}}{\mathrm{nTT}}$ & 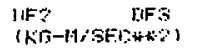 & BII1 & ant & 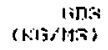 & FI Ans \\
\hline
\end{tabular}
10.005
$30.29 \quad 28.54$
694.43
0.045.
$-6.7 \quad-0.075$
$-0.047 \quad-0.028 \quad 957.184 \quad 1001.100$
4. . $\rightarrow$ rite

ANALYSIS DF DATA

\begin{tabular}{|c|c|c|c|c|c|c|c|c|c|c|c|c|}
\hline \multirow{2}{*}{ TIME } & \multirow{2}{*}{$\begin{array}{l}\text { FluIno } \\
\text { TEMP }\end{array}$} & \multirow{2}{*}{$\begin{array}{c}\text {-TTURB } \\
\text { FTW }\end{array}$} & VFLCICI I Y Y-- & \multirow{2}{*}{ FHHIF } & \multirow[b]{2}{*}{ תזt } & \multirow[b]{2}{*}{ MÄFT } & \multicolumn{3}{|c|}{ 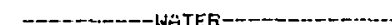 } & \multicolumn{3}{|c|}{ - } \\
\hline & & & FTA & & & & DENS & VFI & MAFW & nENS & Vil & MAFW \\
\hline (SEC) & (CEL) & (M/SFC) & (MT/SFE) & (KG/MH+3 & & $(K, T ; B F C)$ & $(1,0,1+4+3)$ & (M,EEC) & ( $r:\{i, E F(i)$ & (K:Cishlatis) & (MISFCO & ita, sF( ) \\
\hline $10.00 \mathrm{~A}$ & 30.27 & -0.193 & $-10.1 \% 0$ & 981.725 & 0.000 & -0.995 & 994.736 & -0.281 & $-1, .330$ & 0.000 & 11.000 & 0.000 A WTH \\
\hline & & & & & & & & & & & & \\
\hline $20.00 \mathrm{E}$ & $27.0 \%$ & 30.29 & 28.69 & 694.43 & 0.045 & $-\dot{c} \Omega . \dot{c}$ & -0.064 & -0.0147 & $-1,0.42$ & c.5. 1451001 & 1000 & Sillte \\
\hline
\end{tabular}

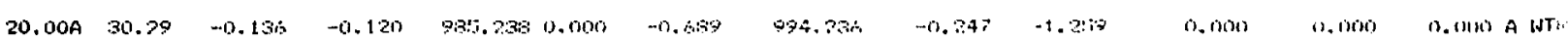

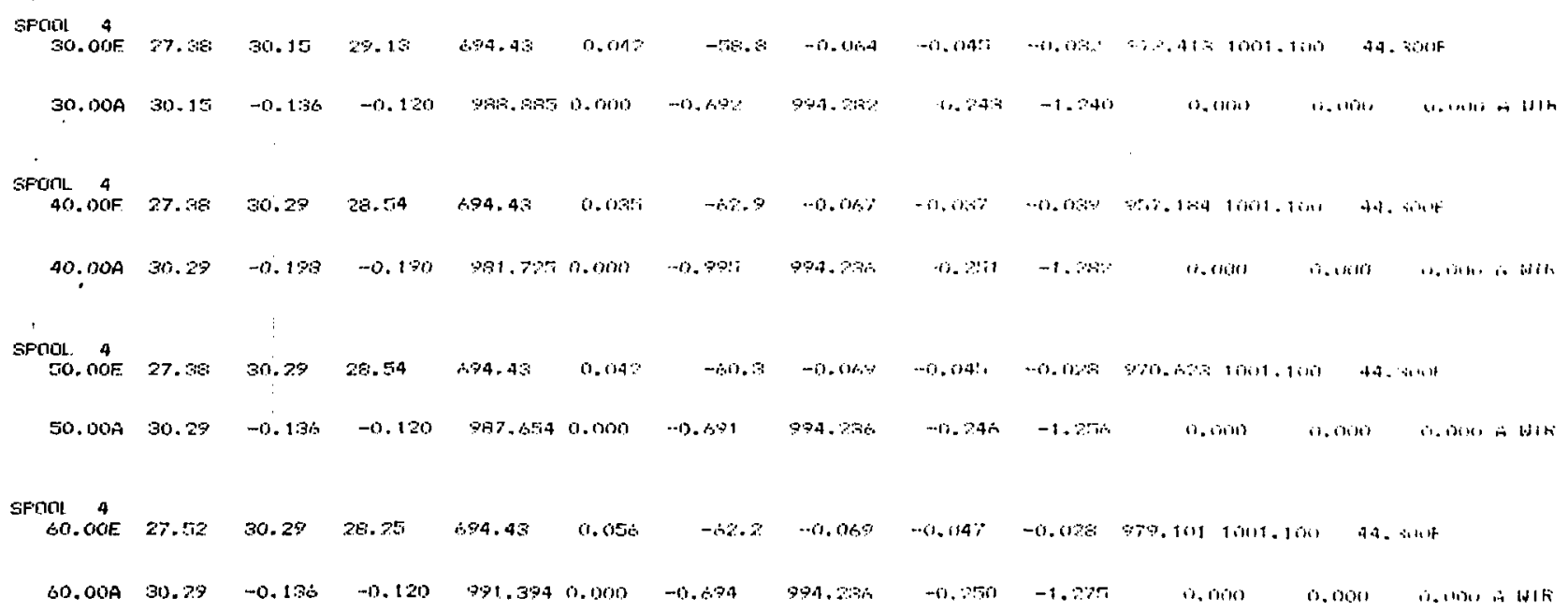




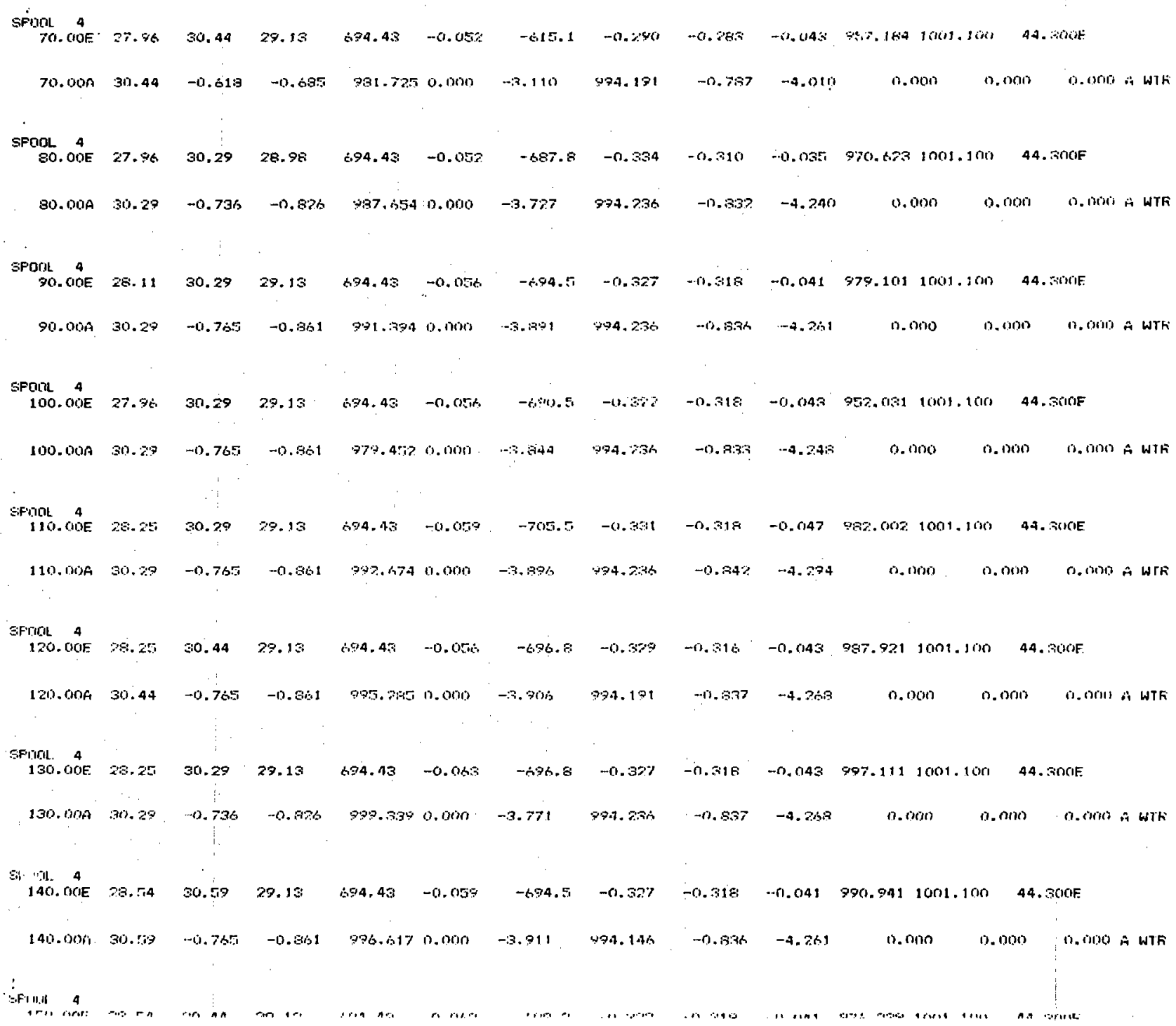




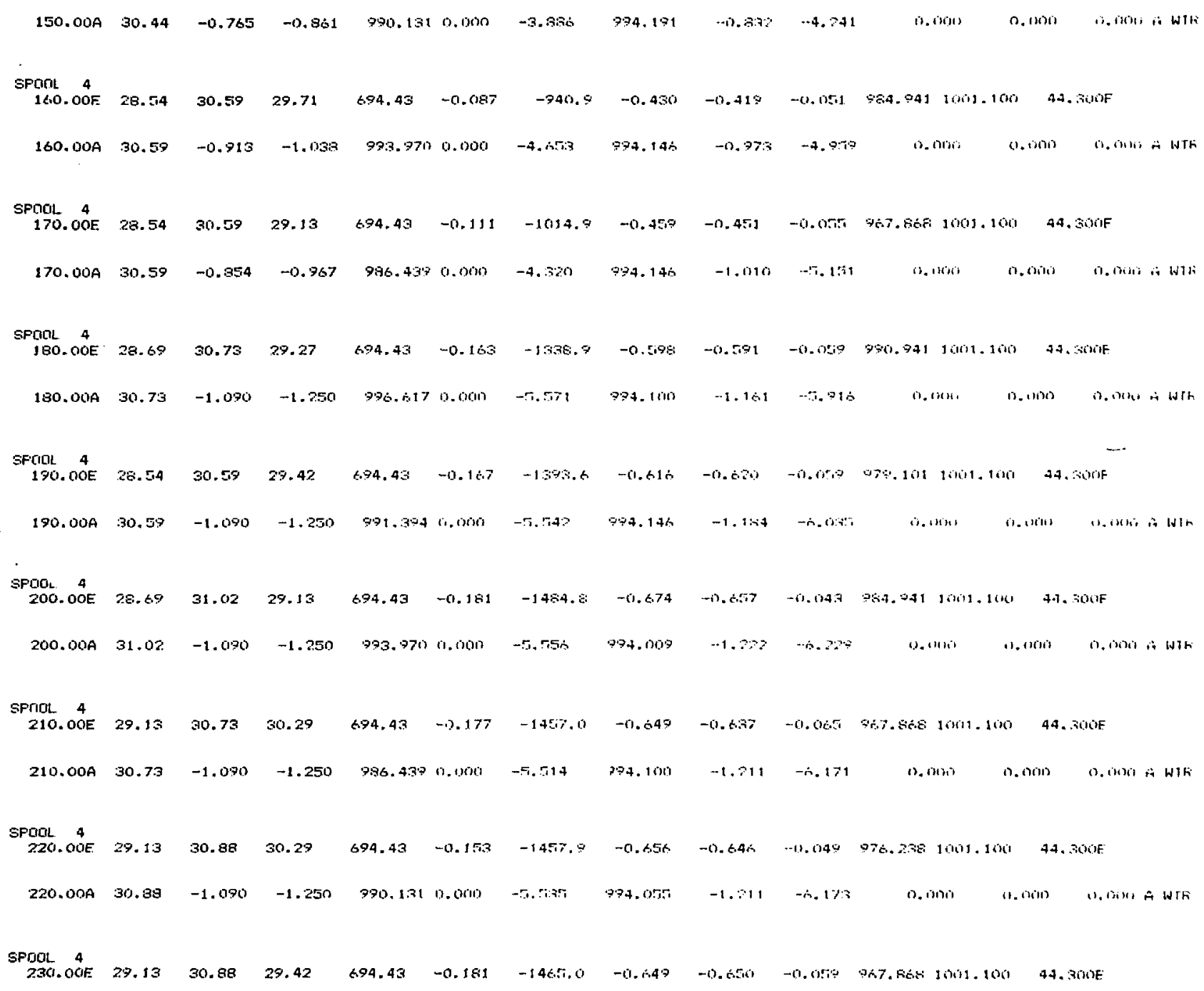




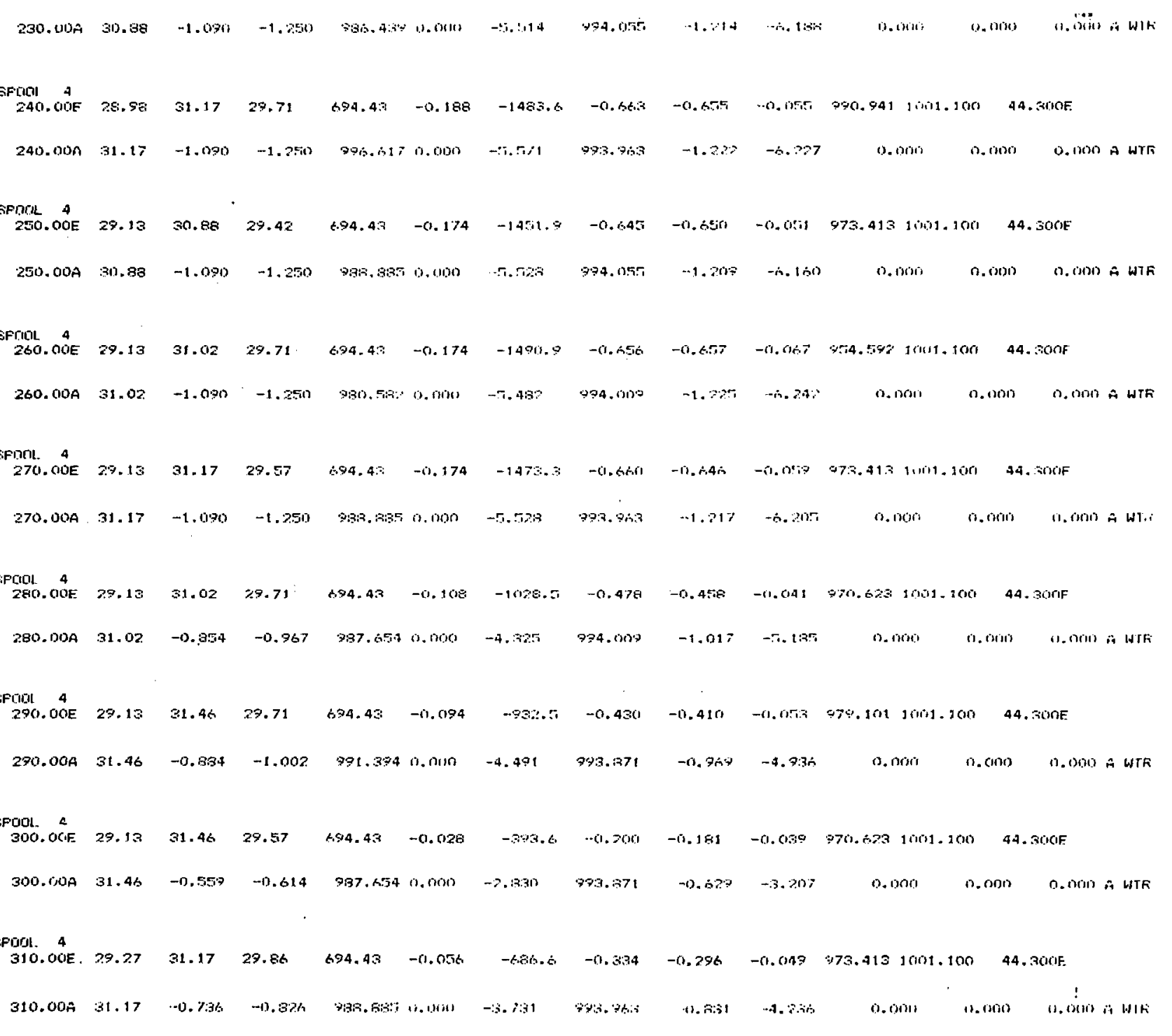




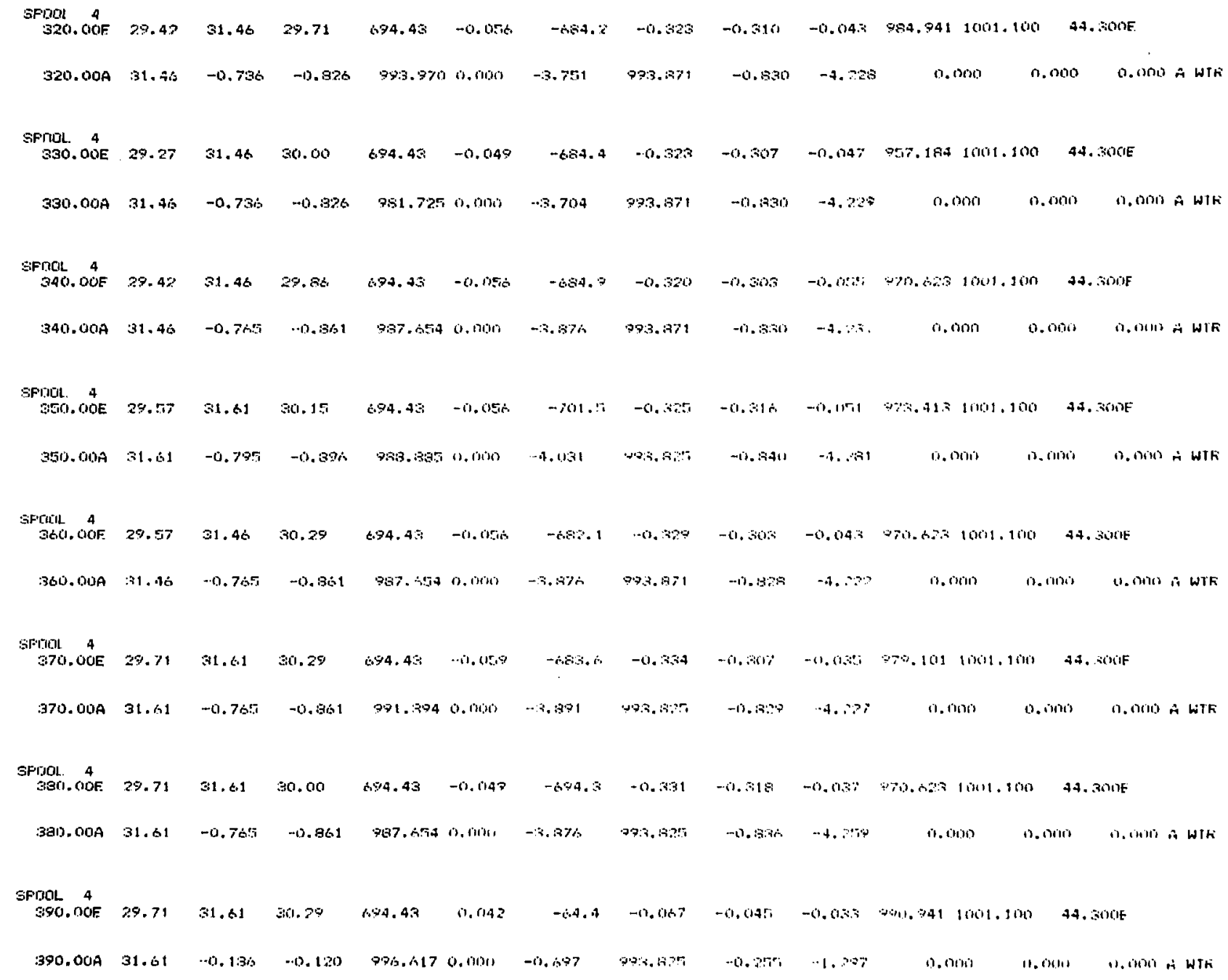




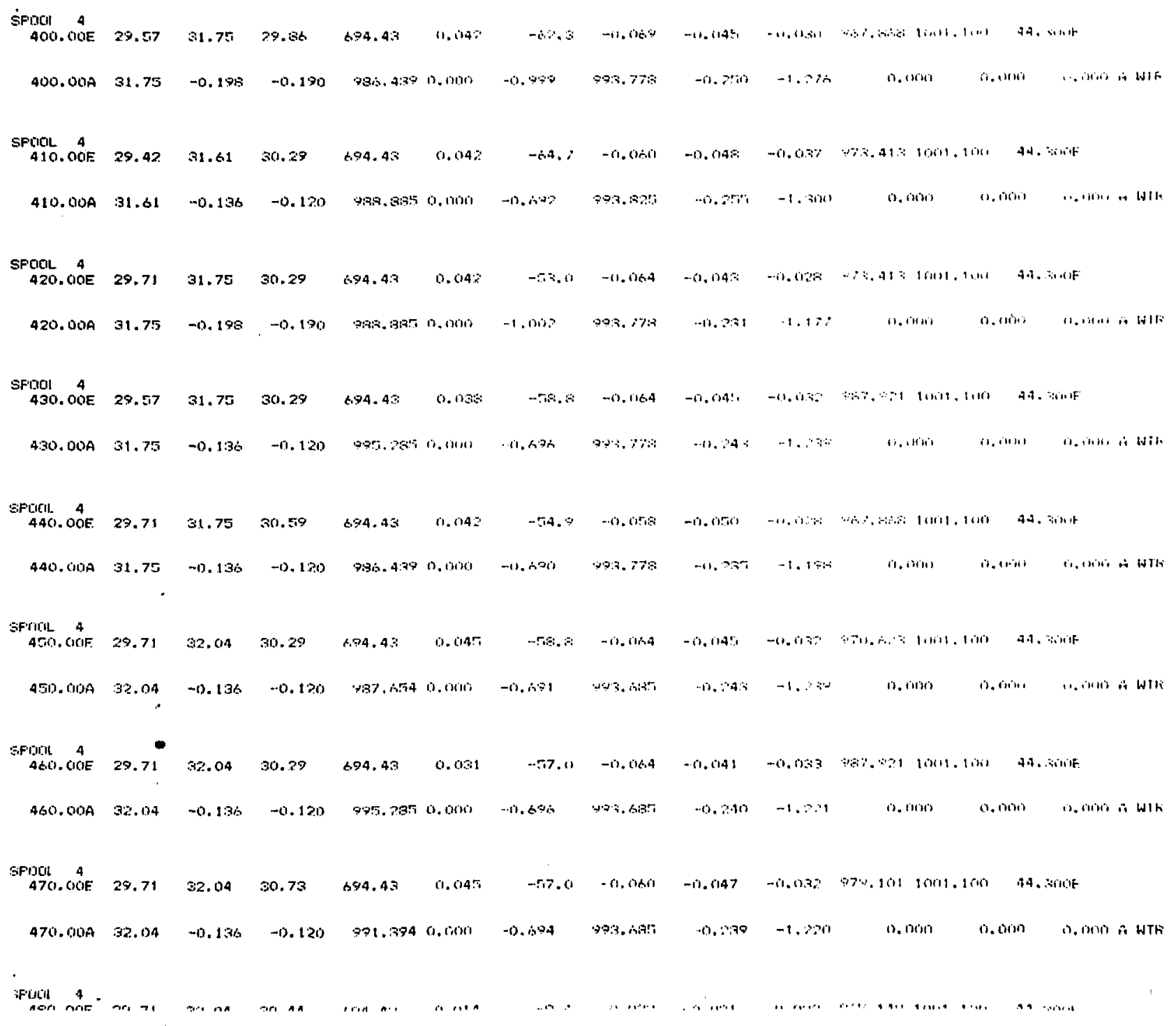




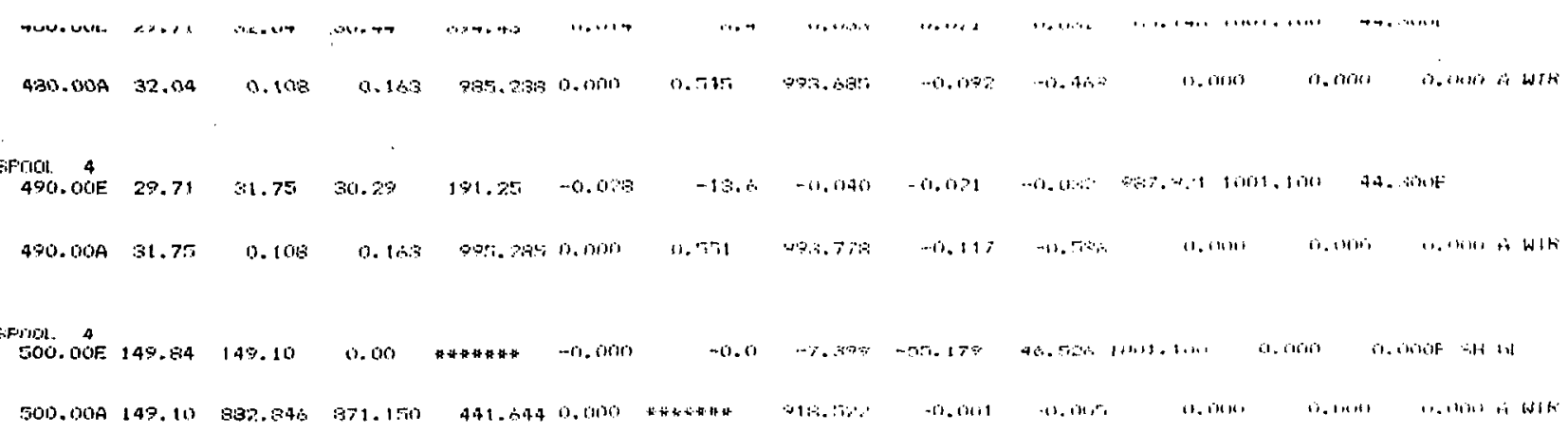


ENGINEERINO UNITS CQNUERSION RESULTS

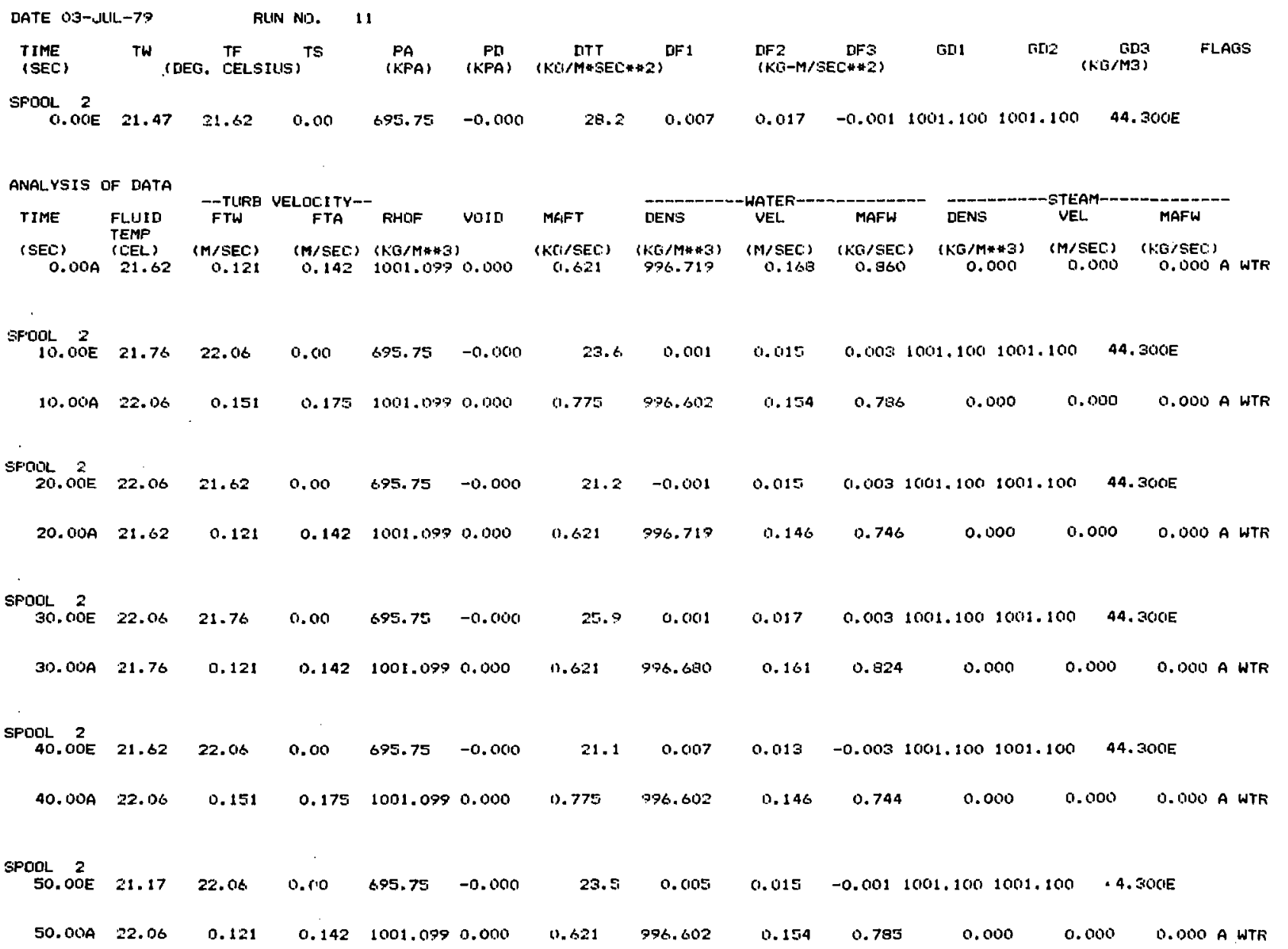




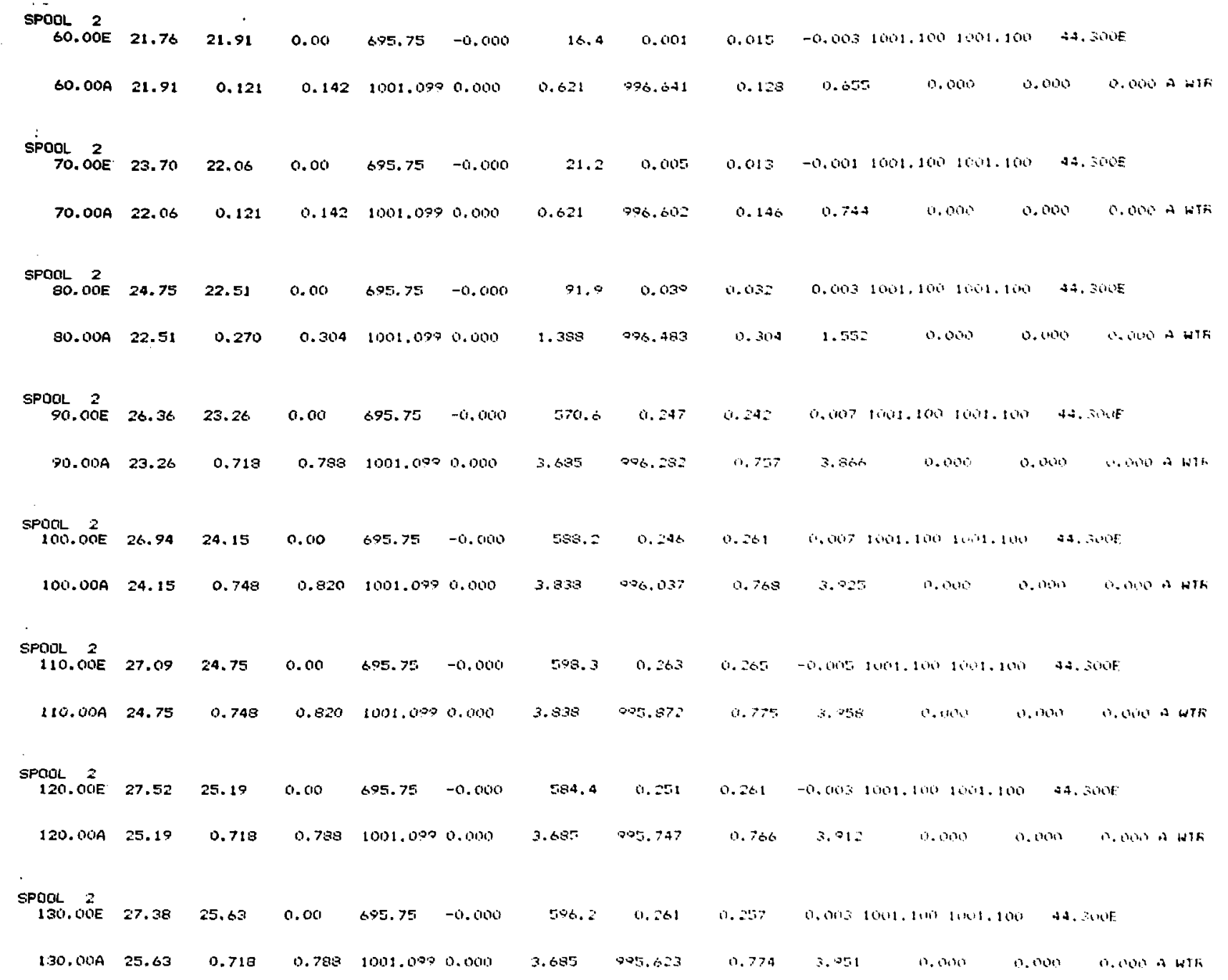

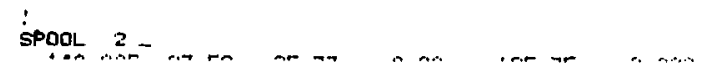




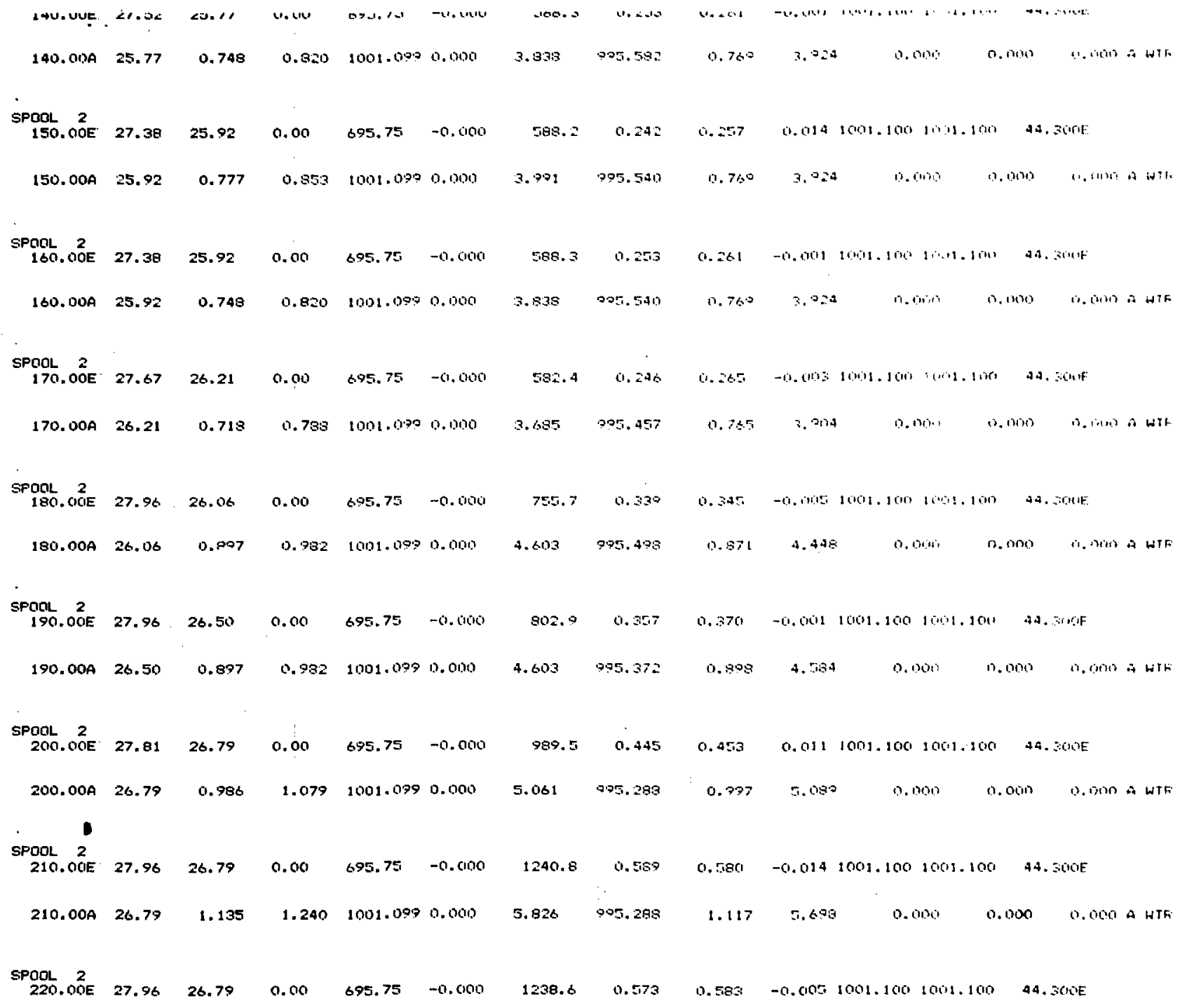




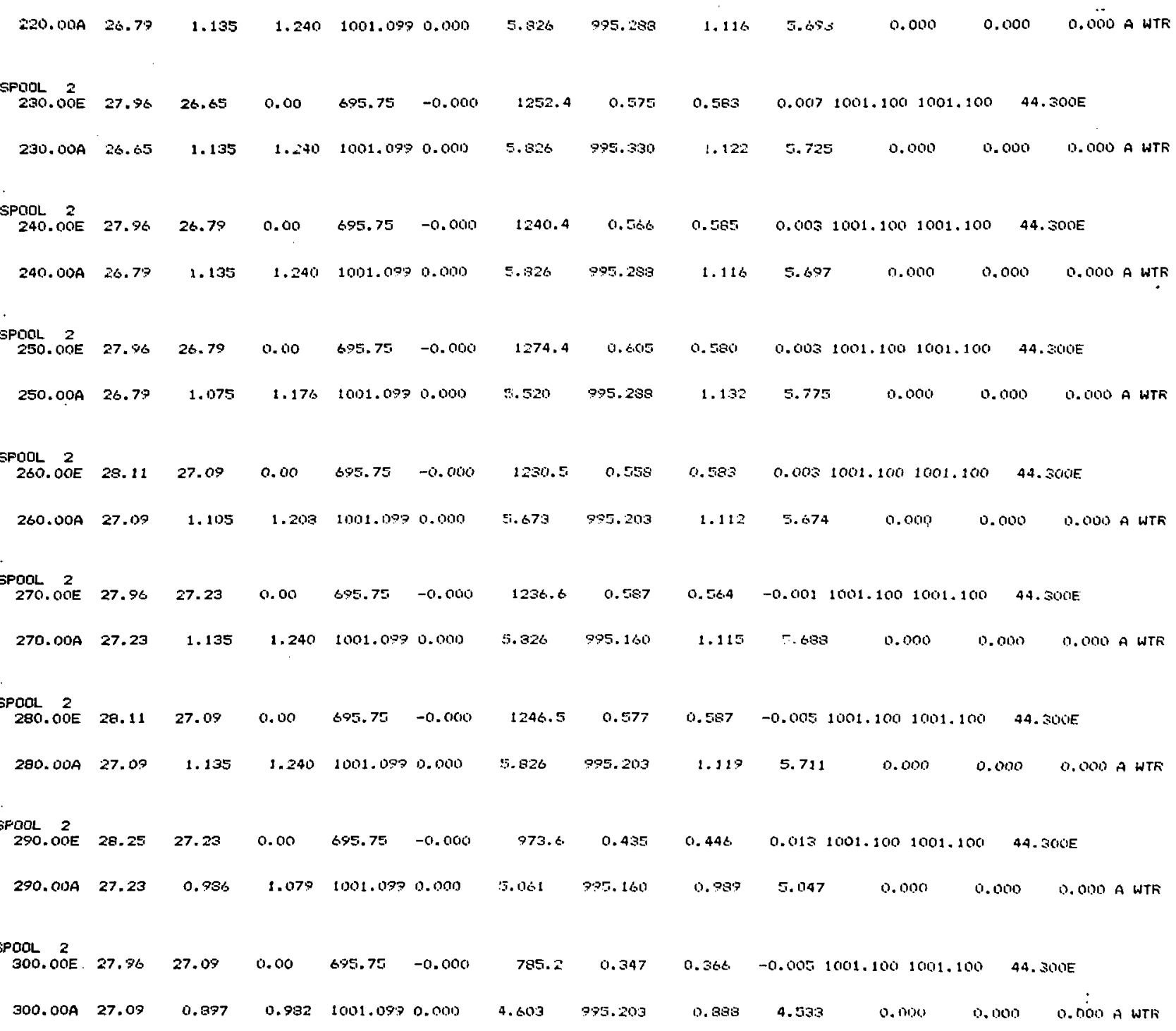




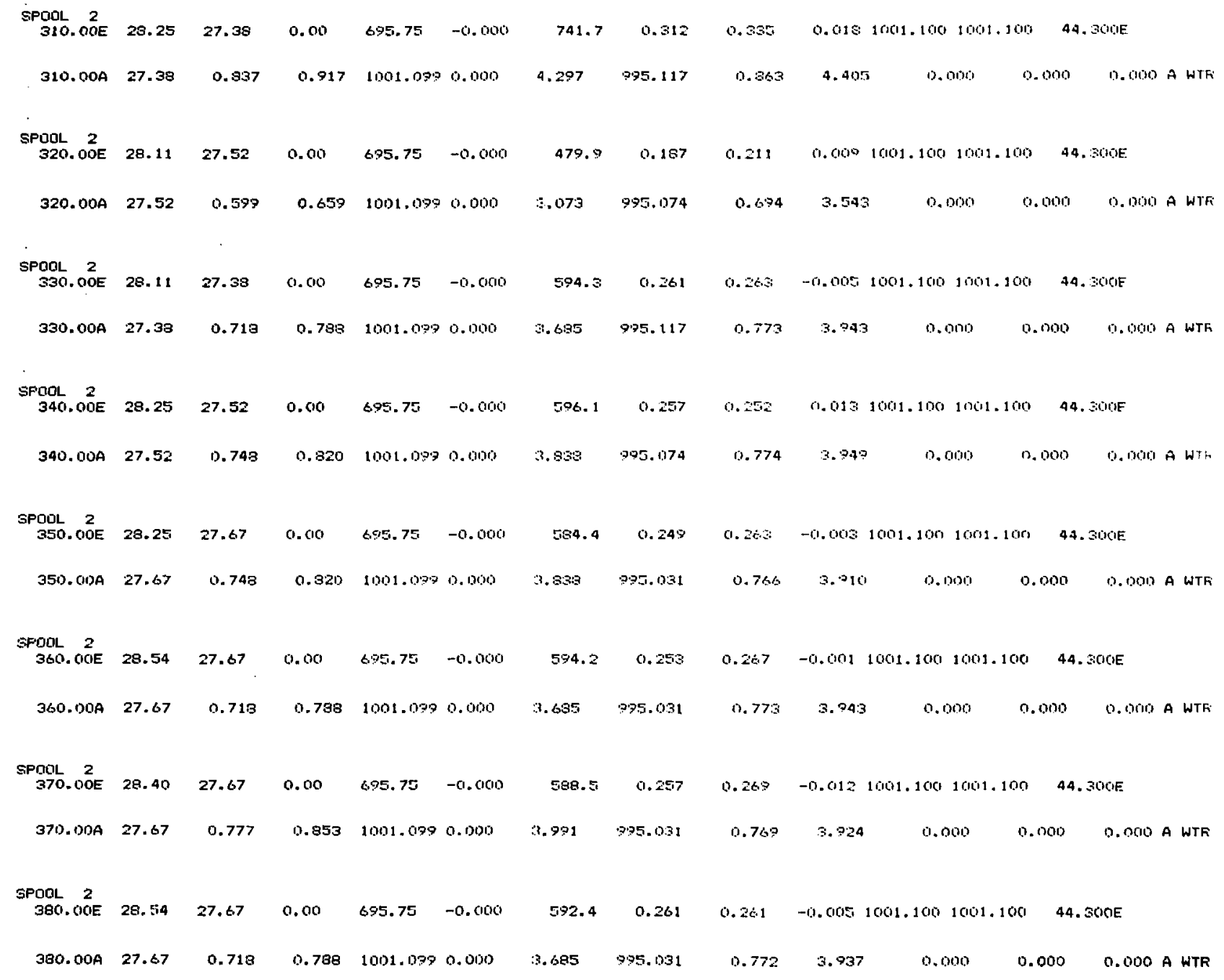




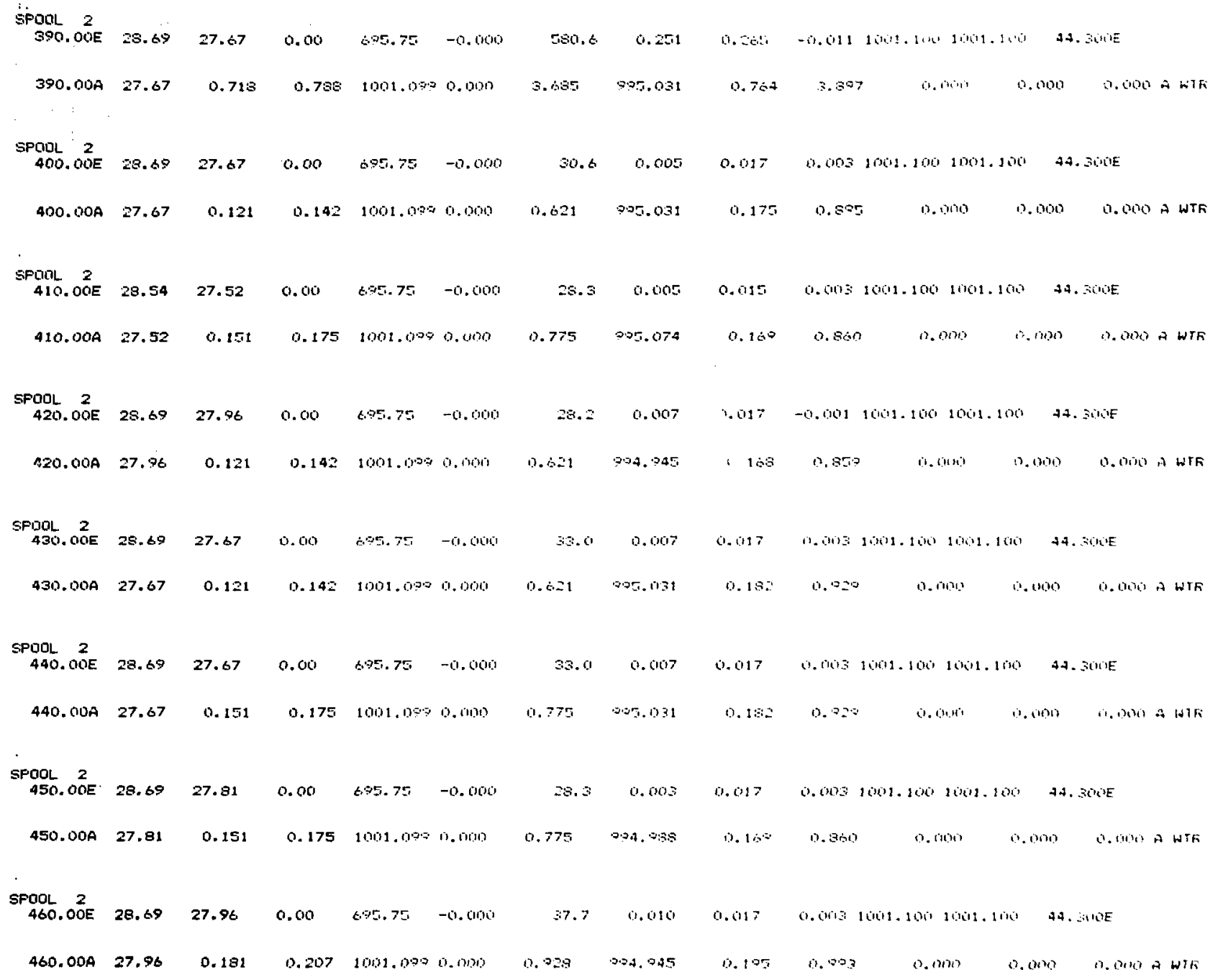

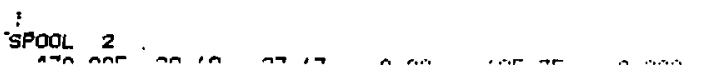




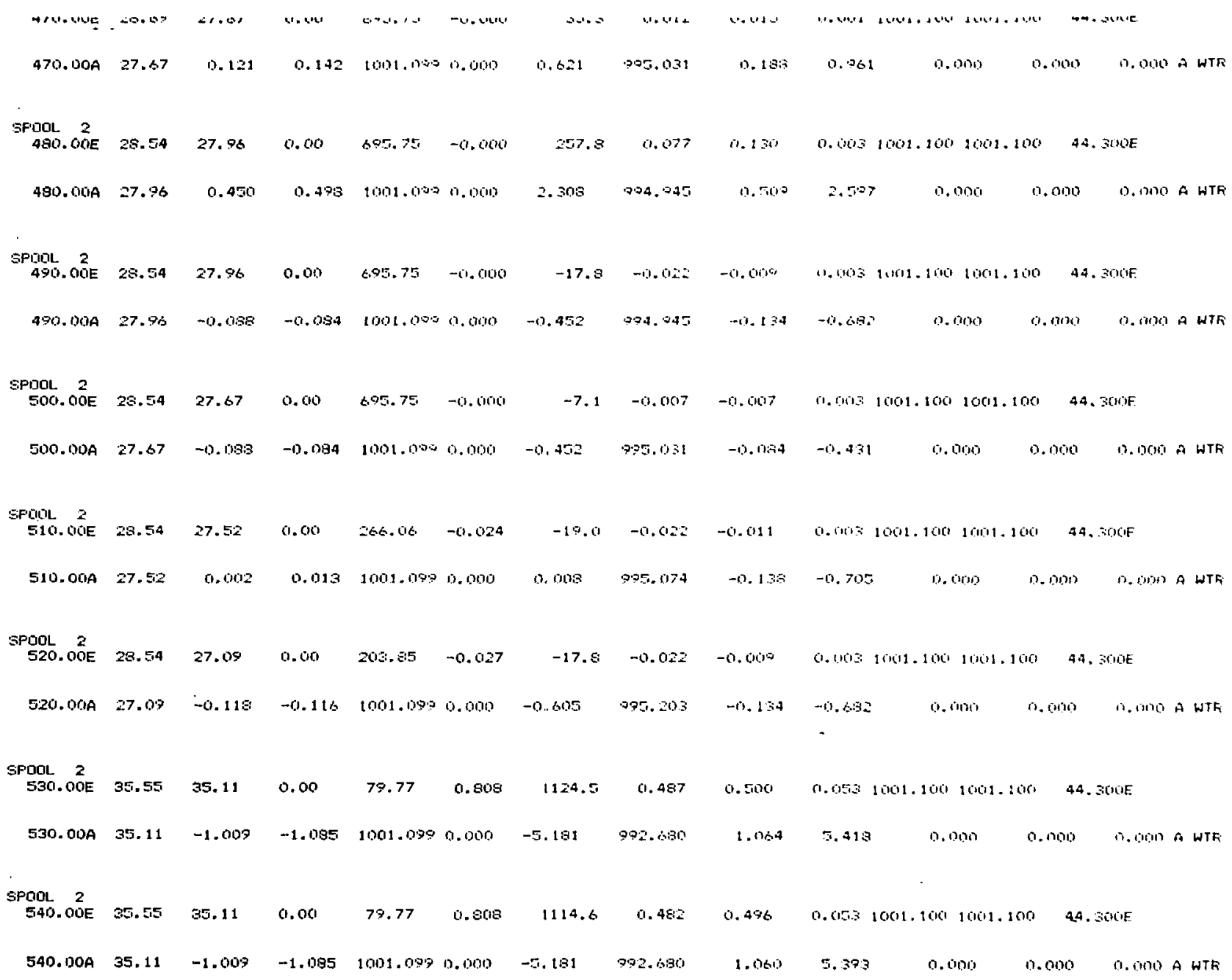


ENGINEERING UNITS CONUERSION RESULTS

DATE O4-UUL-7Q RUN NIT. 12

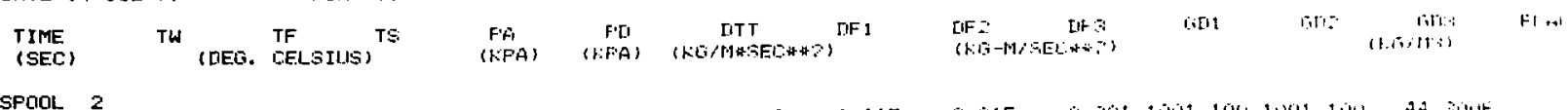

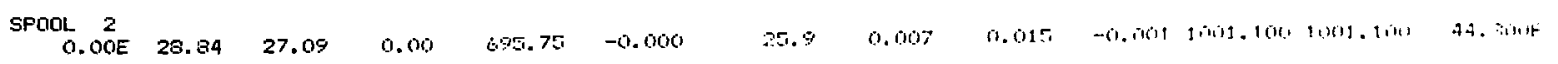

ANALYSIS DF DATA - TURE

(SEC) TEMP

FTW FTA RHIIF VOLI

MAFT

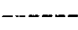

WATEF-D...................

THFW:

0.00A $27.0 \%$

M/SEC) (M/SEC) (KO,MH+B)

(M/SEL) (KOHAH+B)
0.142 1001.090 0.000

(KCOCEEC)

O. $\rightarrow 21$

(IA)

(H:E/EF+)

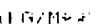

(II) क.

i. 1 is 1

a. cints

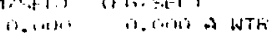

SPQUL 2

10. OOE

28.98

27.36

$0.00 \quad 65.75 \quad-0.000$

40.1

o. $11 \%$

O. i19:

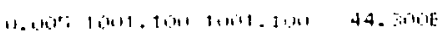

11).00A 27.38

0.121

$0.142 \quad 1001.0000 .000$

$0.401 \quad 00.117$

1. $.011 .11 \%$

is mint

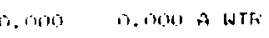

\&

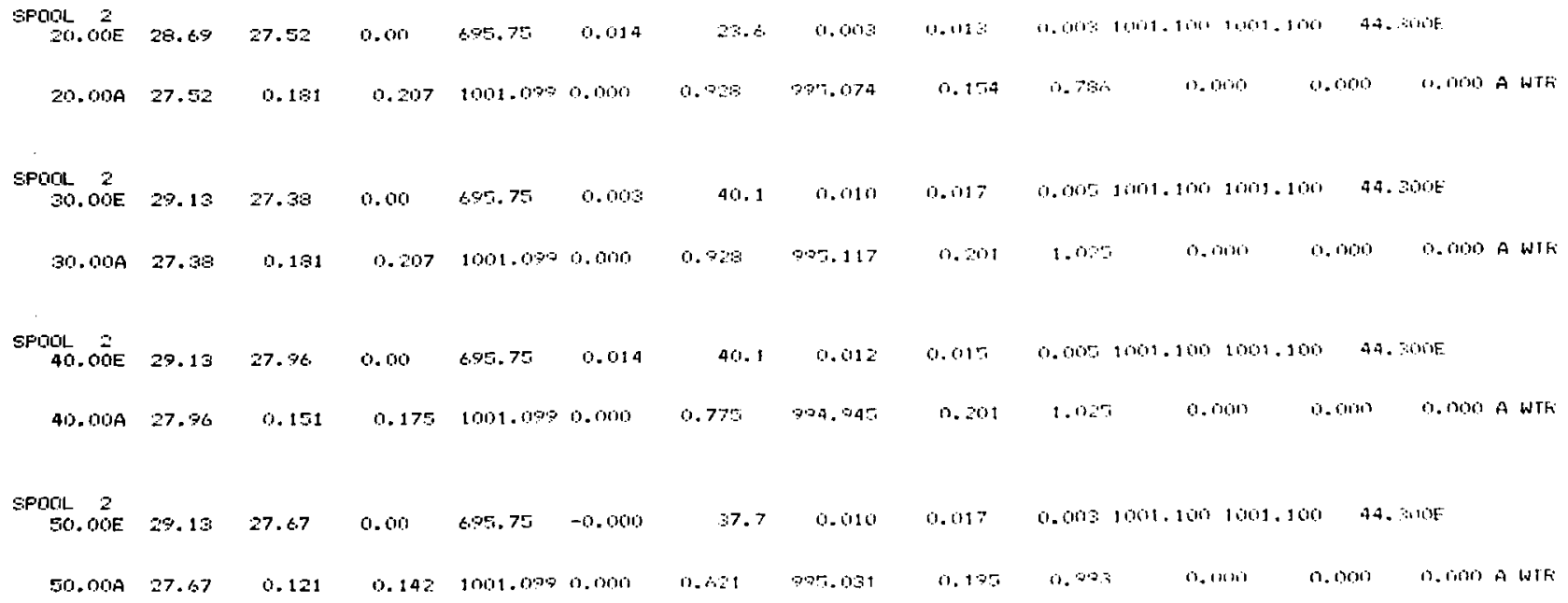




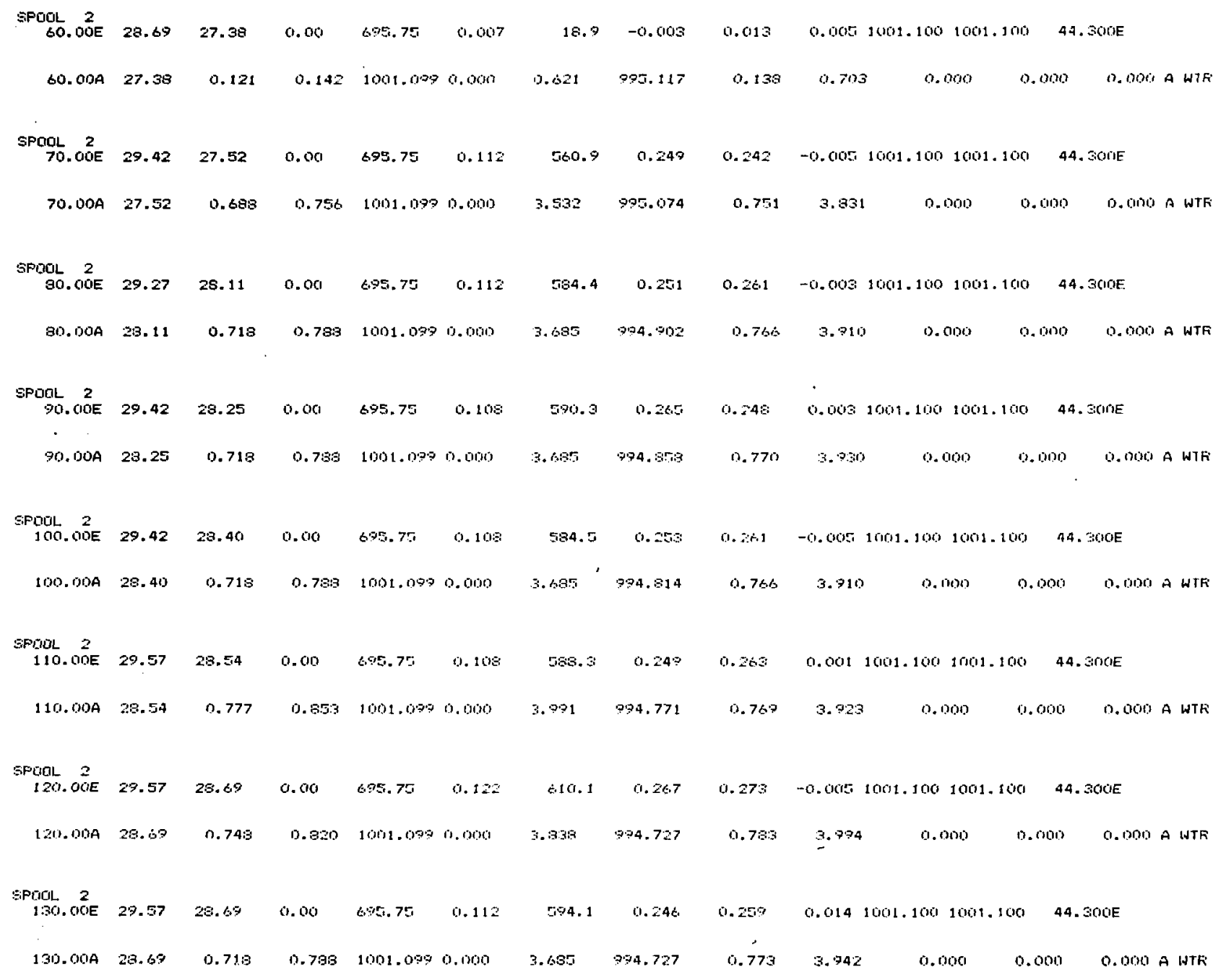

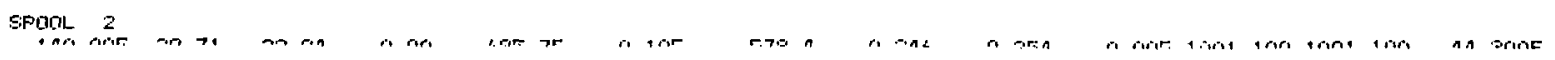




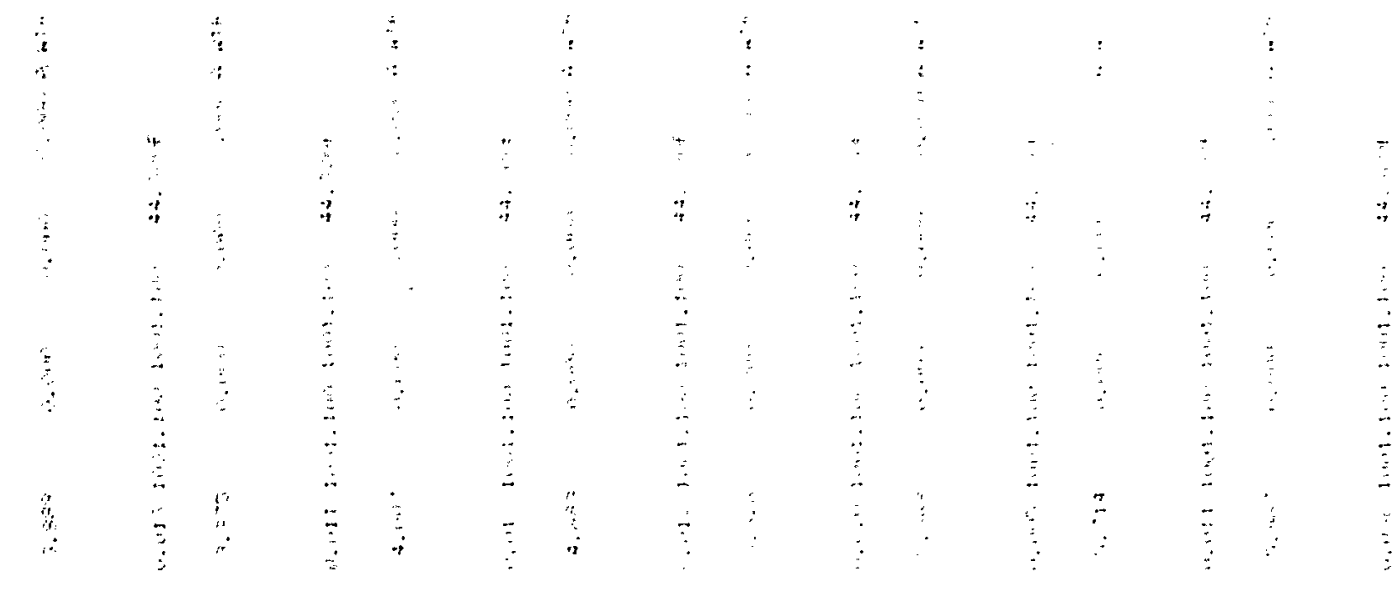

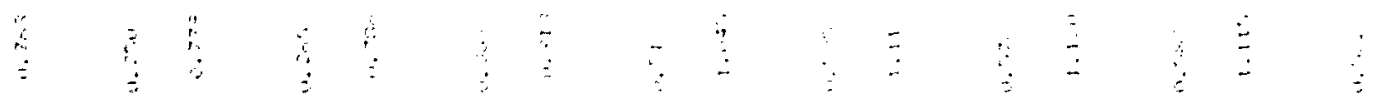

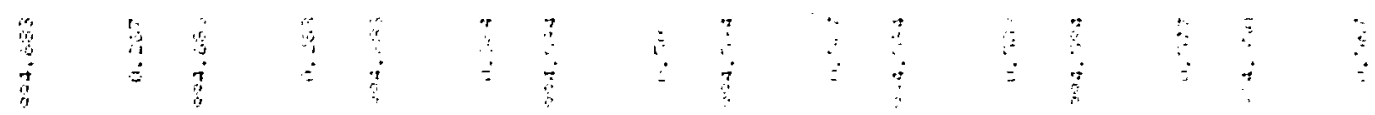

3.

ไै।

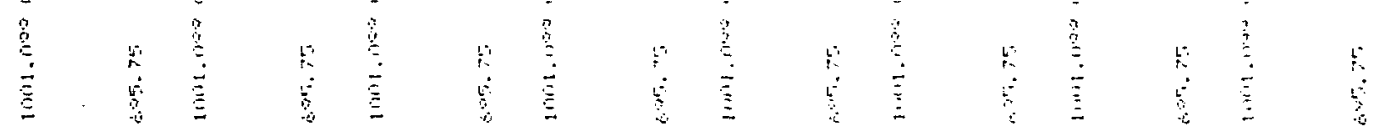

ได้

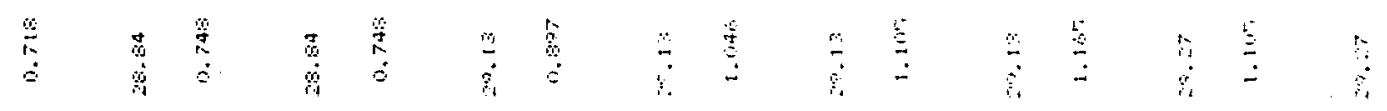

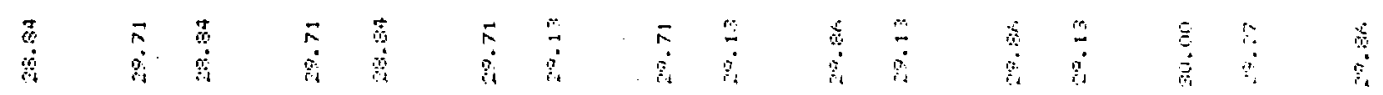

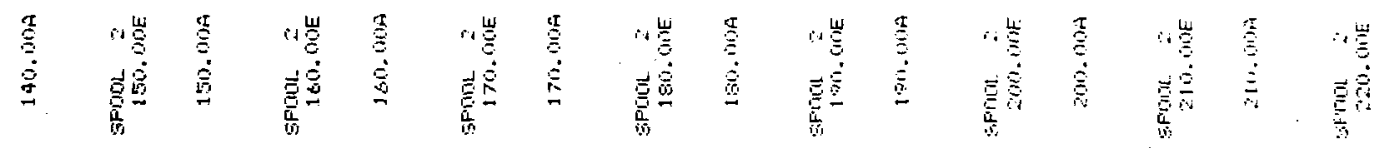




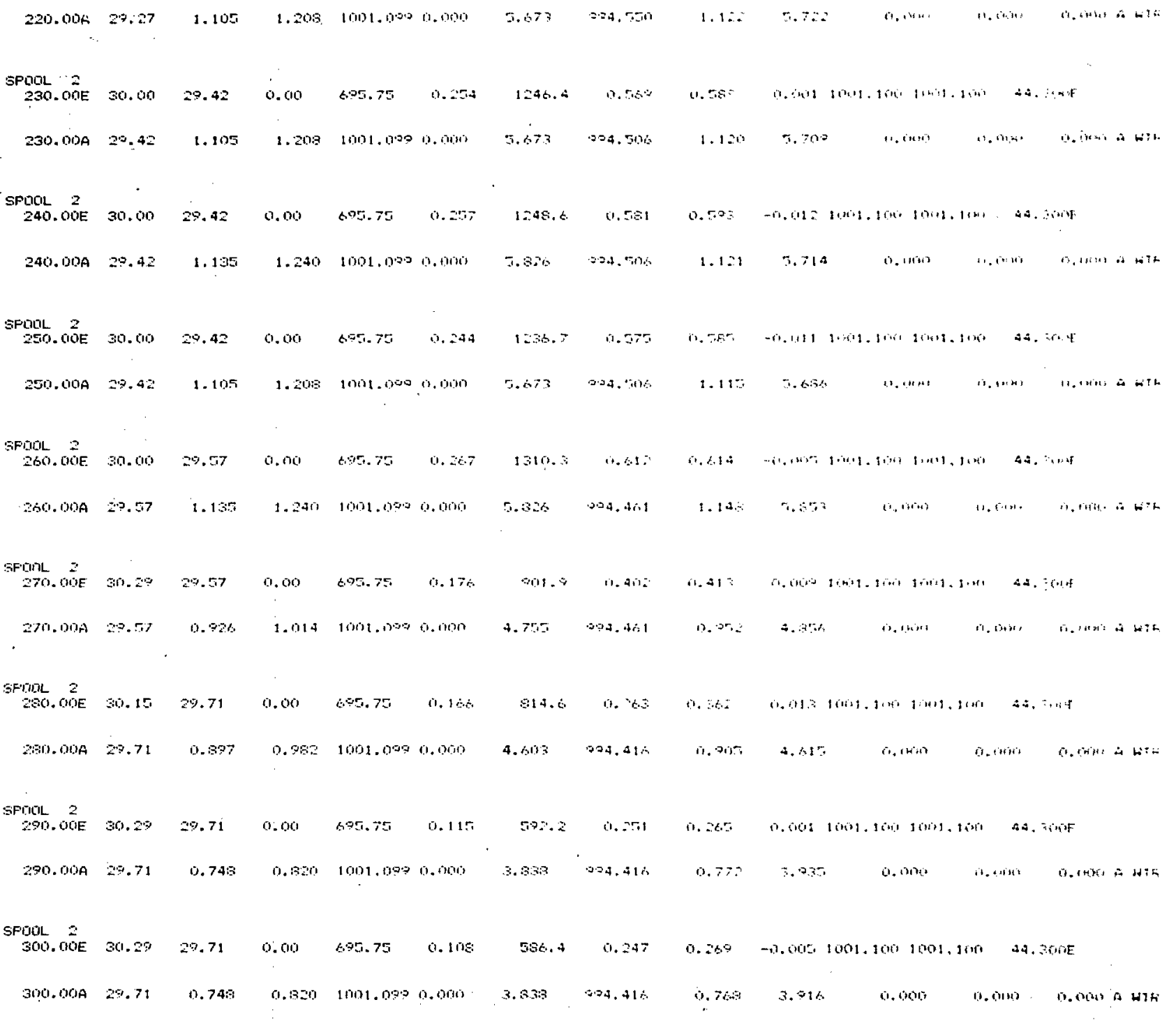




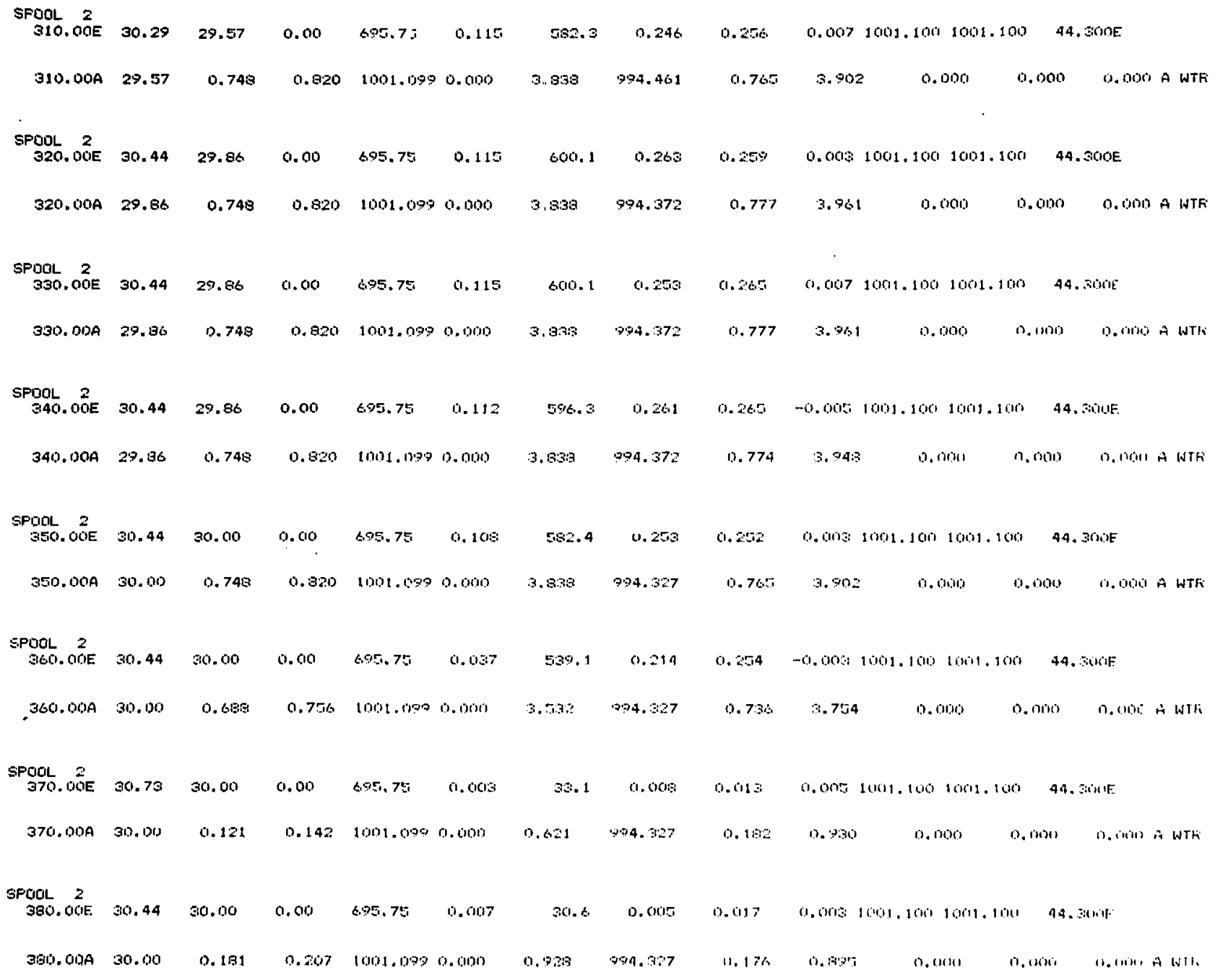




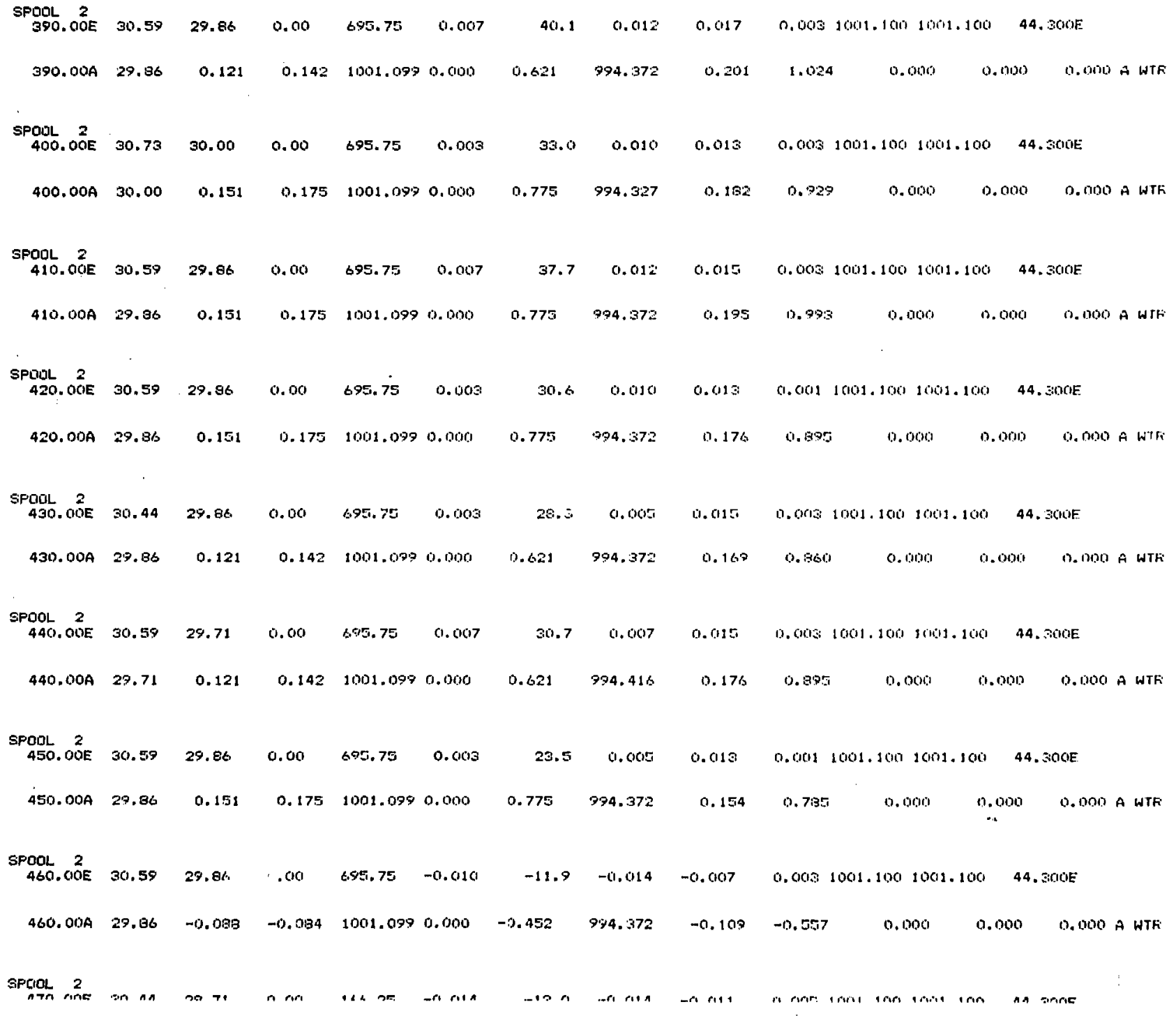




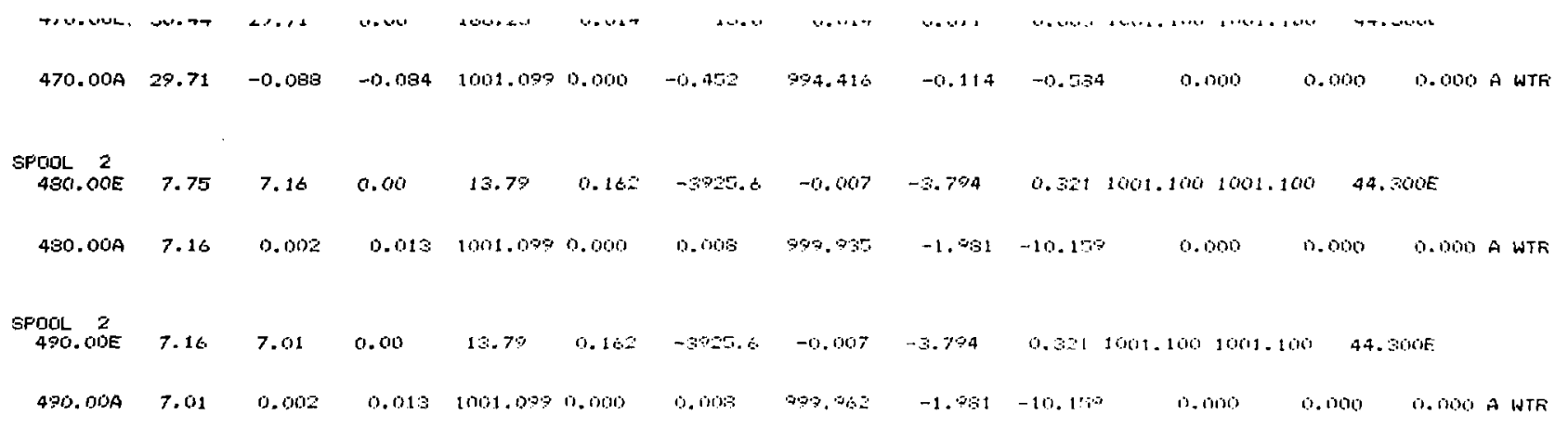




\section{ENOINEERING UNITS CONUERSION RESULTS}

DATE D4-.BLL-7: RUN NO. $1: 3$

TIMF
(SEG)

ANALYSIS OF DATA

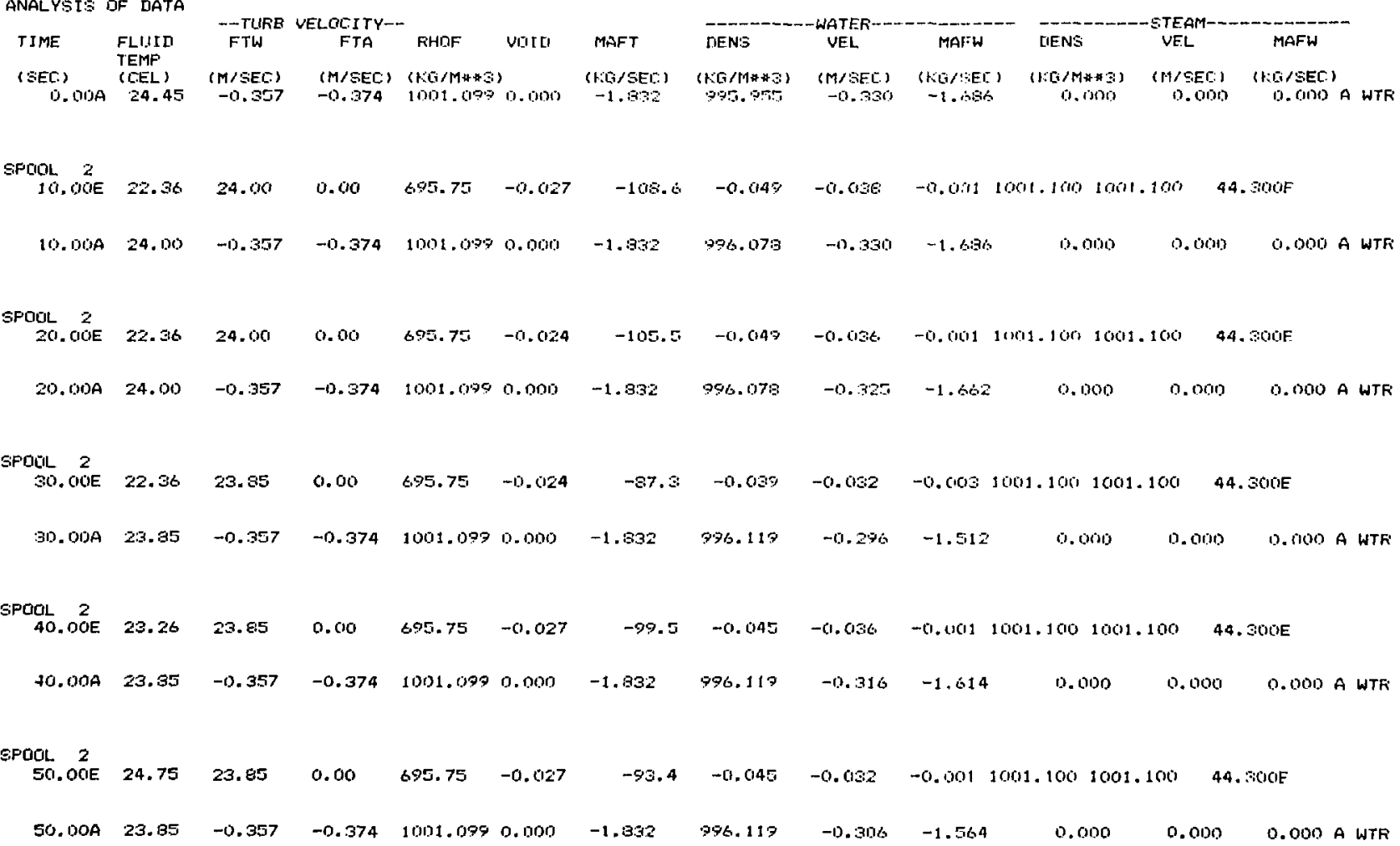




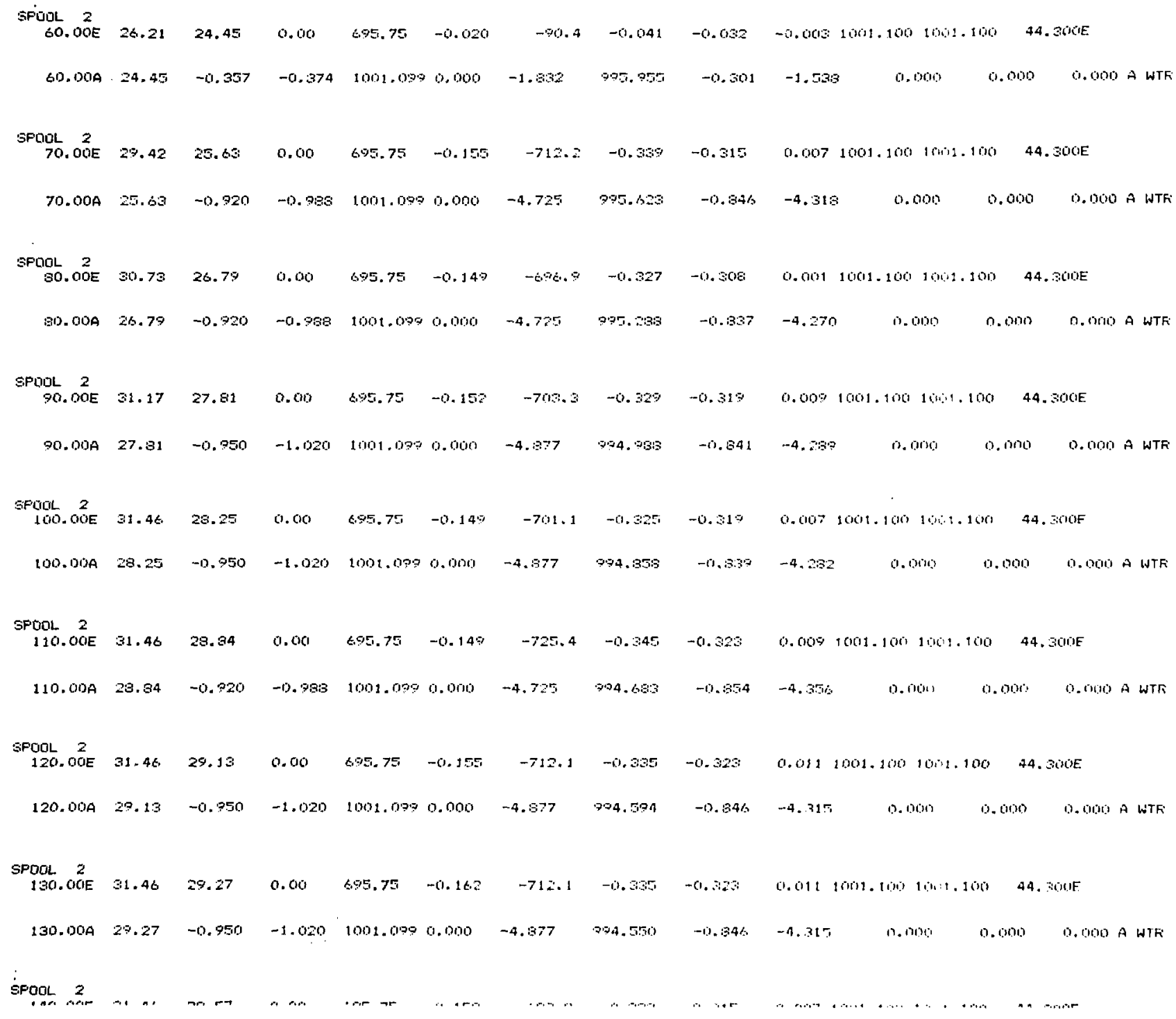




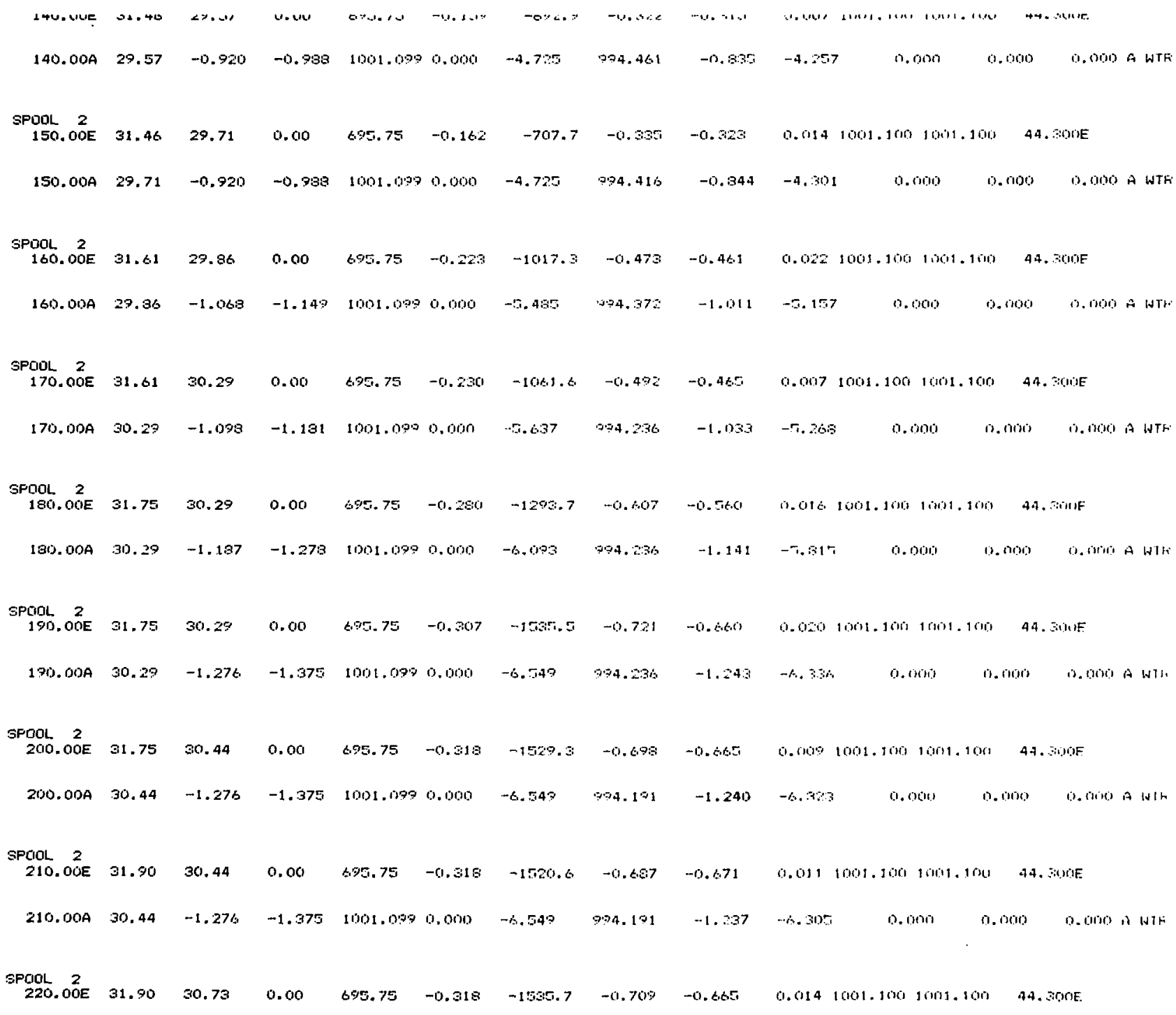




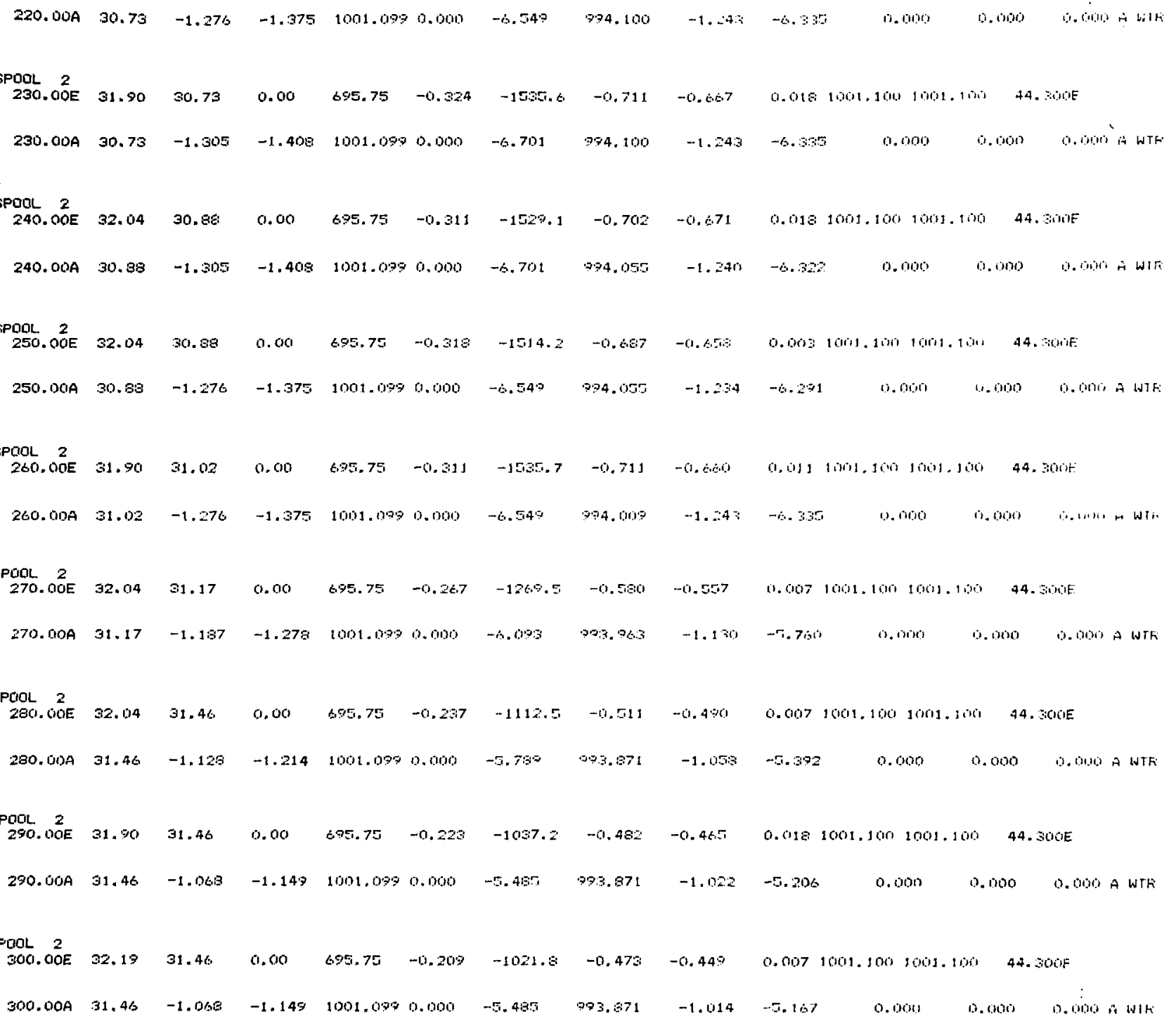




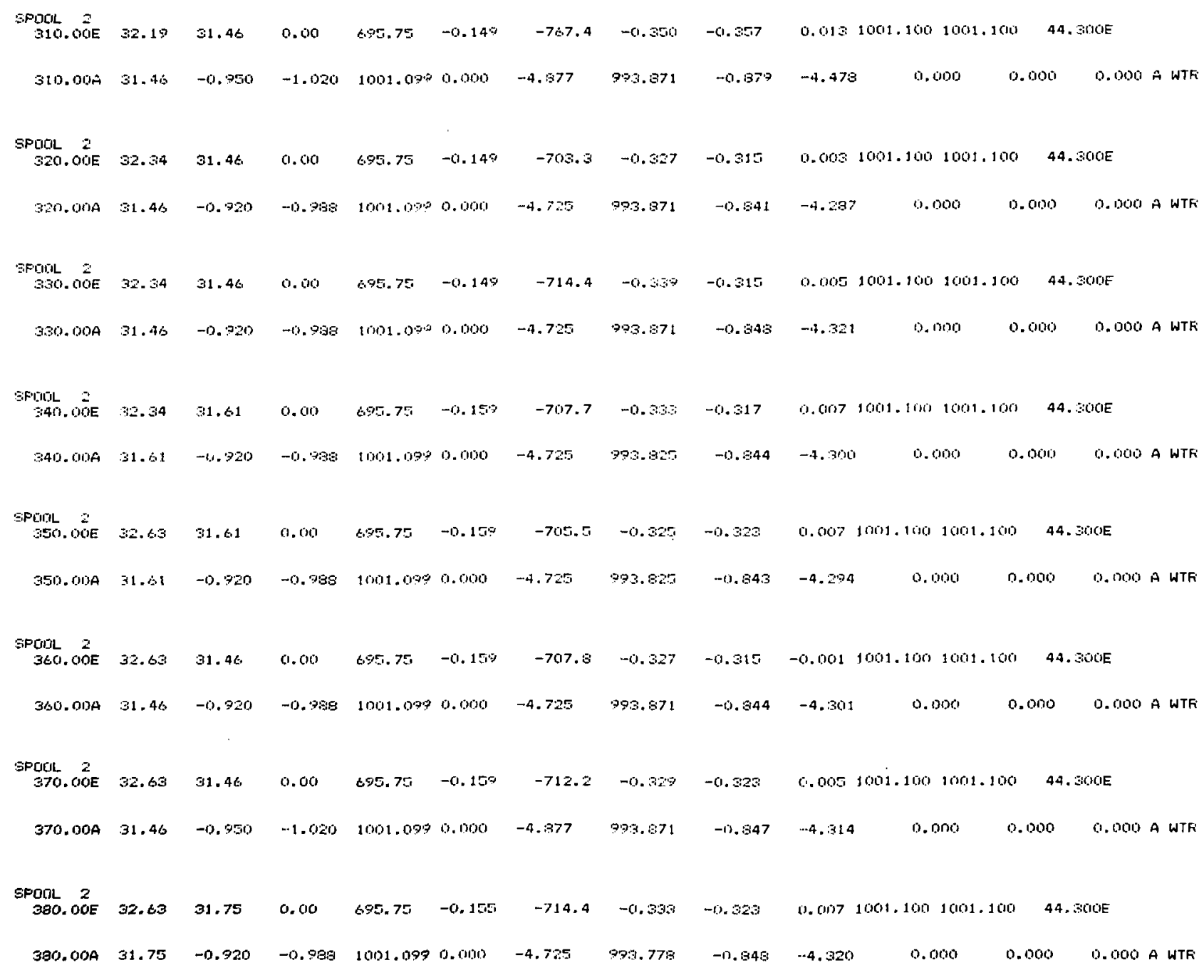


SPOOL 2

390.0

\section{(1)}

SPODL

400.00

400.00

32.63

31.6 .1

0.00

6.5.

SPOOL 2

$410.00 \mathrm{E}$ 32.63

$410.100 A \quad 31.46$

31.46

0.00

$695.75 \quad-0.024$

$-105.6$

$-1.35$

$90,5 \% 7$

SFOOL 2

$420.00 \mathrm{E} \quad 32.4 \mathrm{E}$

$420.010 \mathrm{~A} \quad 31.46$

31.46

$0.00 \quad 605.75 \quad-0.030$

$-111.0 \quad-0.04 \%$

993.971

SPÖLL 2

430. DOE 32.03

$31.6 t$

430.100

31.62

$-0.357$

$0.00-05.75-0.027$

$-0.574 \quad 1001.00000000$

SPIIIL

440.00E $32.63 \quad 31.46 \quad 0.00 \quad 395.750 .024$

440.000

$31.46 \quad-0.357$

$-0.3741001 .0000 .000$

$-1.63$

GPQDL 270.006 \$2.63 $31.46 \quad 0.0$

450. $000 \quad 31.46 \quad-0.355$

460.010

$\begin{array}{llllll}460.000 & 31.46 & -0.357 & -0.374 & 1001.000 & 0.000\end{array}$

GPUOL 2 


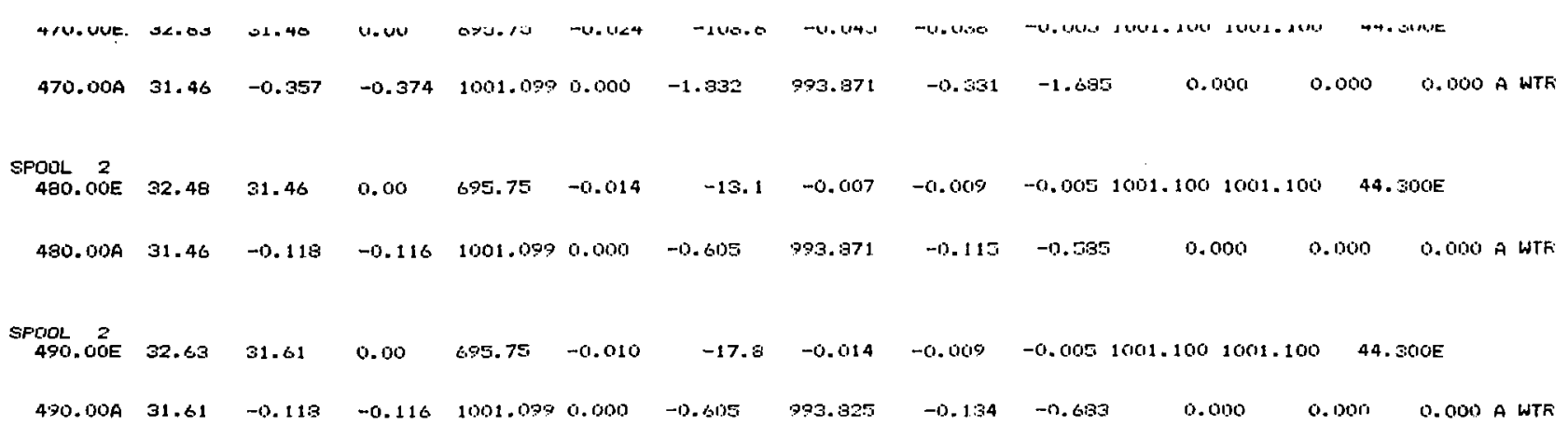




\section{ENGINEERING UNITS CONVERSION RESULTS}

DATE O4-D.NIIL-79 RUN NU. 14

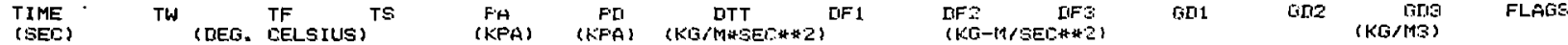

SFGOL 2

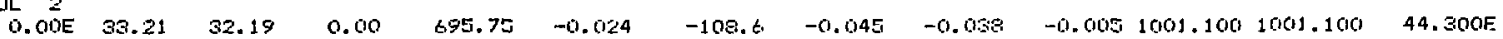

ANALYSIS IF LATA

\begin{tabular}{|c|c|c|c|c|c|c|c|c|c|c|c|c|}
\hline & & & LEIE:I TY-- & & & & & JATEF & - & & EAlT- & \\
\hline TIME & $\begin{array}{l}\text { FLUID } \\
\text { TEMF }\end{array}$ & FTW & FTA & FHIDF & vota & MAFT & RENS & VEI. & MAFW & DENS & VEL & MAFW \\
\hline (SEC) & (CEL) & (M/SEC) & $\begin{array}{l}(M / S E C) \\
-0,374\end{array}$ & $\begin{array}{l}\text { (ho/Matis) } \\
1002,000\end{array}$ & 0,000 & $\begin{array}{c}(K G / S E C) \\
-1 / 33^{\circ}\end{array}$ & $\begin{array}{c}(K G / M+43) \\
973,638\end{array}$ & $\begin{array}{r}(M / S E C) \\
-0.331\end{array}$ & $\begin{array}{l}\text { (1:GASEC) } \\
-1.694\end{array}$ & $\begin{array}{c}(100 / 9+4) \\
0.040\end{array}$ & $\begin{array}{r}\text { (M/SEC) } \\
0.000\end{array}$ & $\begin{array}{l}(160 / S E C) \\
0.000 \text { A }\end{array}$ \\
\hline
\end{tabular}

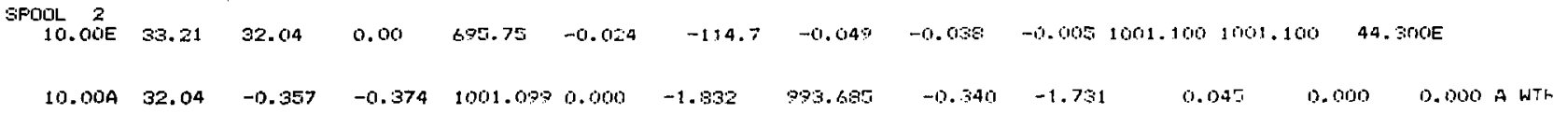

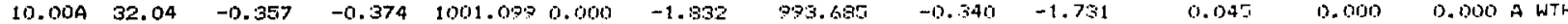

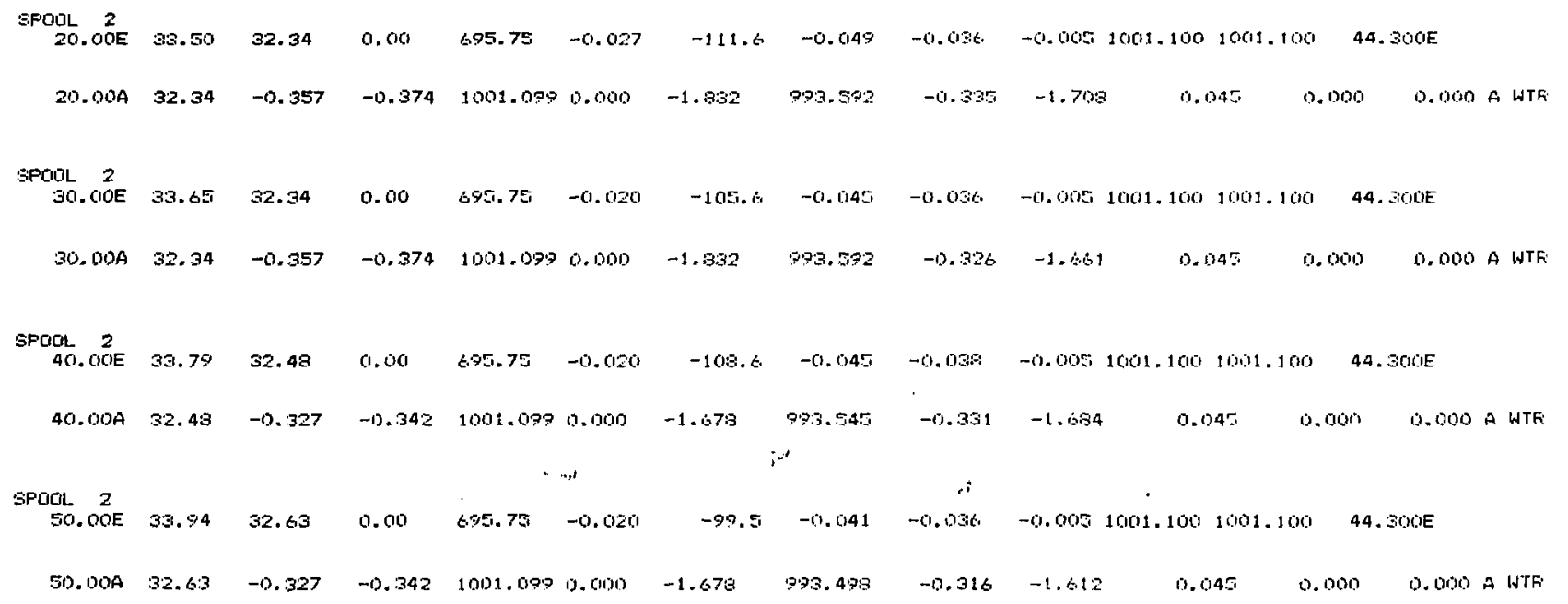


ENGINEERING UNITS CONUERSION RESULTS

DATE 04-IJUL-79 RUN NO. 14

\begin{tabular}{|c|c|c|c|c|c|c|c|c|c|c|c|c|c|}
\hline $\begin{array}{l}\text { TIME } \\
\text { (SEC) }\end{array}$ & TW, & G. ${ }_{\text {CEL }}$ & $(S)^{T S}$ & $\begin{array}{l}P A \\
\langle K P A\rangle\end{array}$ & $\begin{array}{c}F D \\
(K F A)\end{array}$ & $\begin{array}{c}\text { RTT } \\
(K G / M * S E C H+2)\end{array}$ & ]$^{\mathrm{DF} 1}$ & $\begin{array}{l}\mathrm{BF2} \\
\left\langle K^{\prime} 13-11\right.\end{array}$ & $\begin{array}{c}\text { IfF } 3 \\
(0 * 2)\end{array}$ & CiD1 & 602 & $\begin{array}{r}G \mathrm{G} 3 \\
(\mathrm{KG} / \mathrm{MB})\end{array}$ & FL.ACSS \\
\hline $\begin{array}{l}\text { SPAOL } \\
90.00 E\end{array}$ & 34.38 & 33.21 & 0.00 & 695.75 & -0.149 & -659.1 & -0.318 & -0.311 & 0.002 & 1001.100 & 1001.100 & 44.300E & \\
\hline
\end{tabular}

ANALYSIS OF DATA

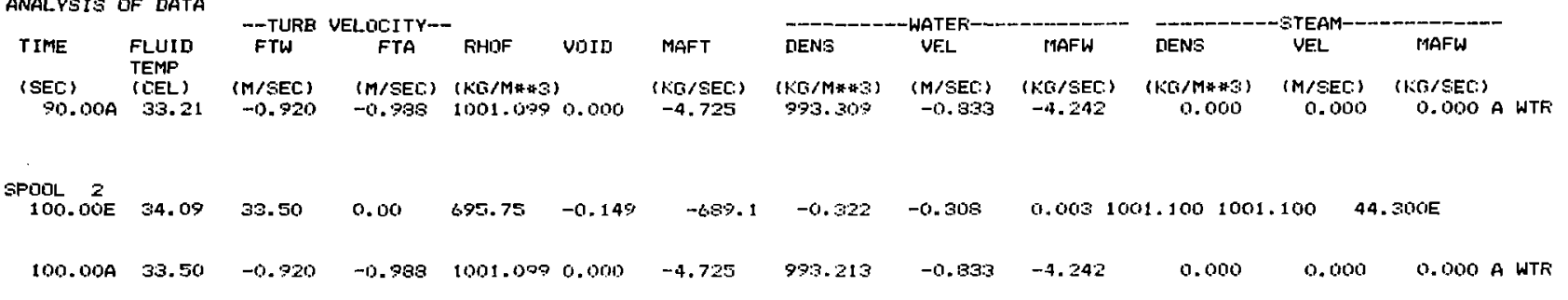

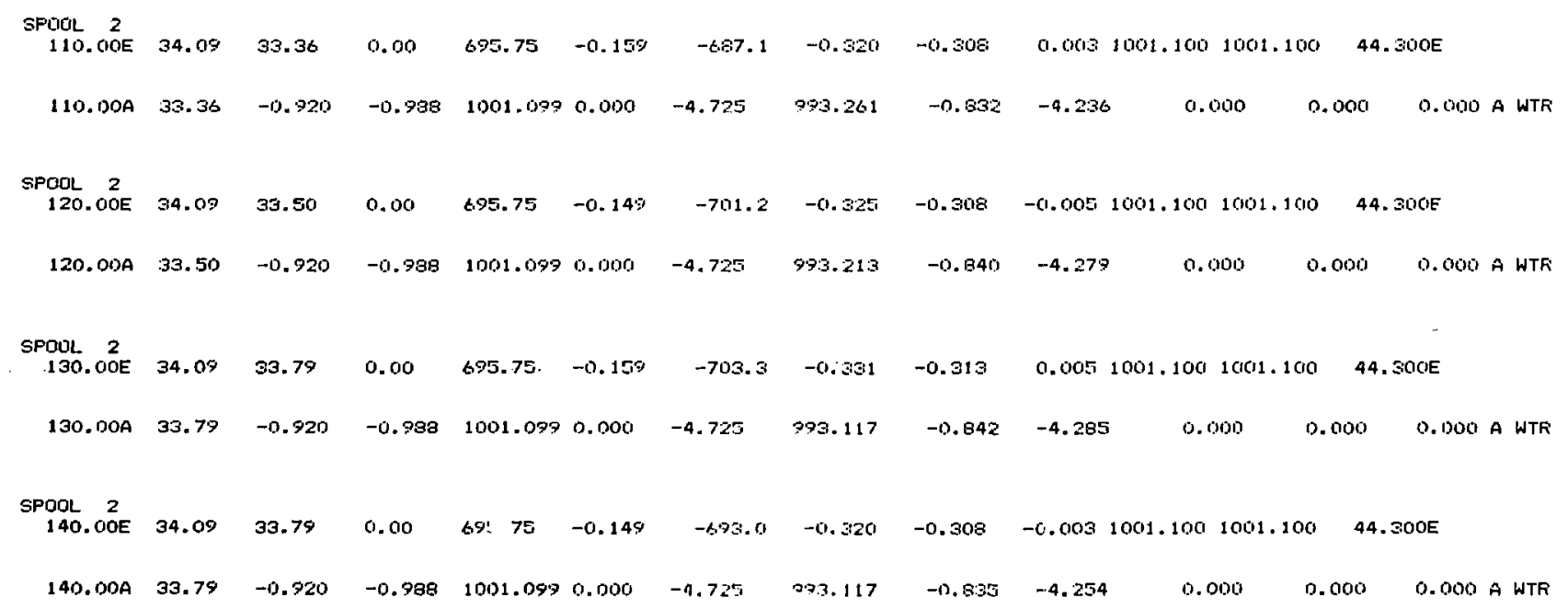




\section{ENVINEERING UNITS CONVERSION RESULTS}

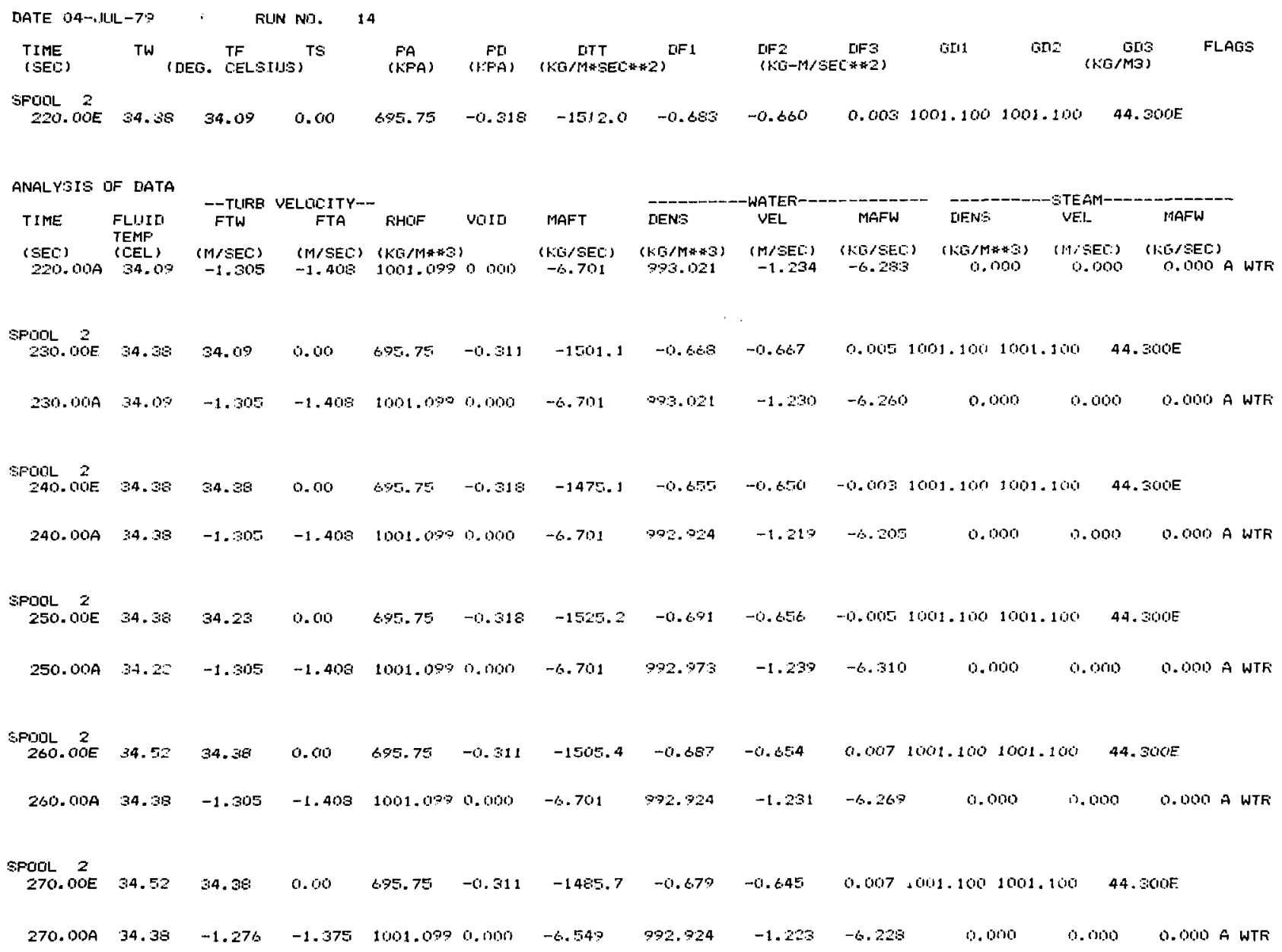


ENGINEERING UNITS CONVERSIUN RESULTS

DATE O4-JUL-78 RUN Na. 14

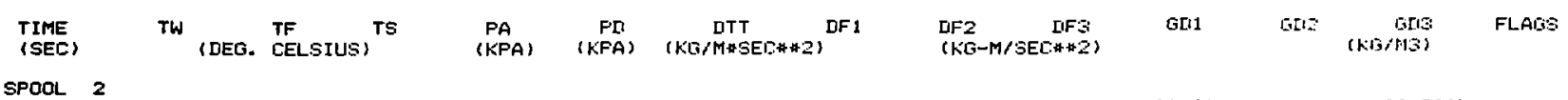

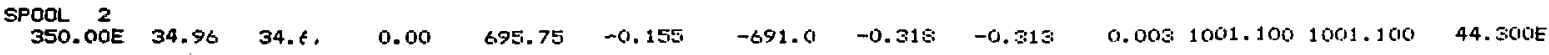

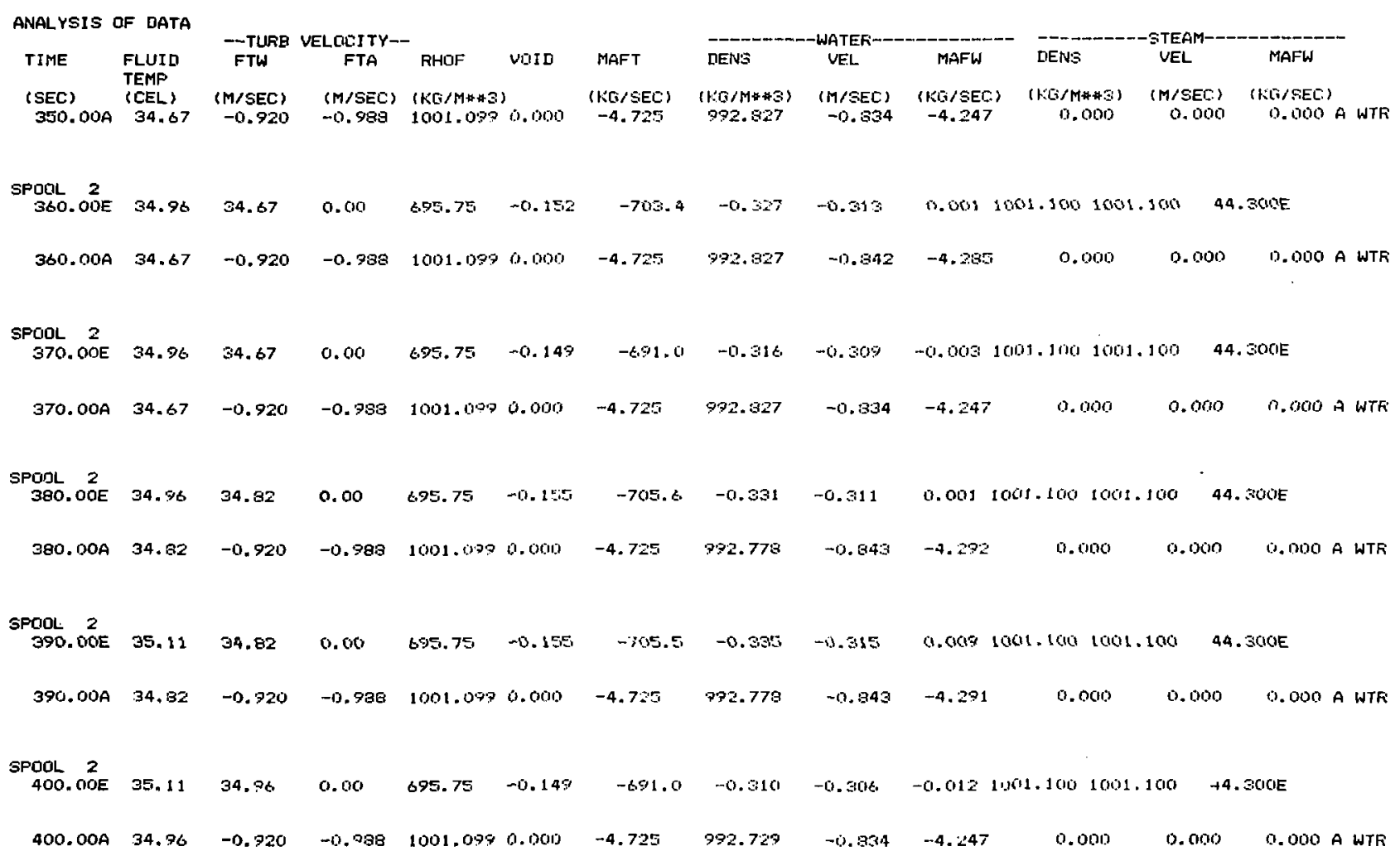


ENGINEERING LINITS CONVERSION RESULTS

DATE O4-JUL $\rightarrow$ RLIN NO. 24

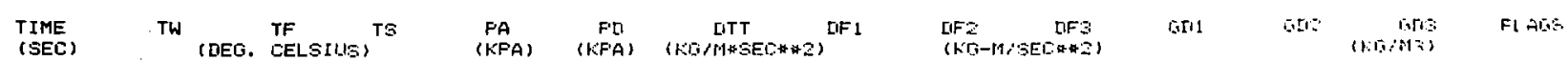

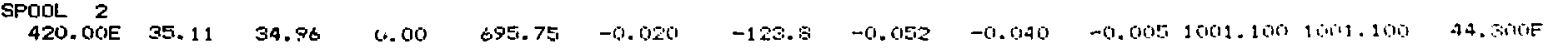

ANALYSIS OF DATA

\begin{tabular}{|c|c|c|c|c|c|c|c|c|c|c|c|c|}
\hline & & --TUFEE & VEL_DCITY〜- & & & & 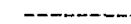 & WAT & ....... & & .TFi & (-.... \\
\hline TIME & $\begin{array}{l}\text { FLUIID } \\
\text { TEMP }\end{array}$ & FTW & FTA & RHGF & venta & MAFT & IIEN'S & VEL & MAFW & 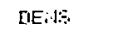 & $\because F L$ & MNFW \\
\hline & (CEL) & $(M / S E C)$ & (M/SEC) & 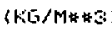 & & (1KG，SEL) & $(1: G / N * * 3)$ & $(M / S E C)$ & 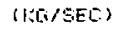 & $1160 / M \times 4+2$, & (MiSE( ) & 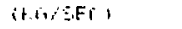 \\
\hline $0.00 \mathrm{~A}$ & 34.96 & -0.327 & -0.342 & 1001.099 & 0.000 & -1.679 & 792.727 & -0.353 & -1.797 & 0.000 & 0.000 & a. onio in \\
\hline
\end{tabular}

SPOOL 2

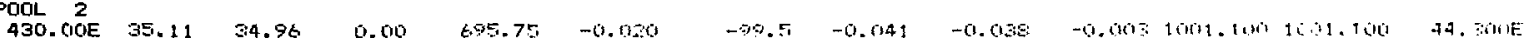

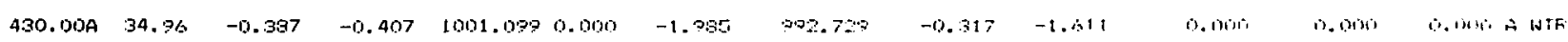

\begin{tabular}{|c|c|c|c|c|c|c|c|c|c|c|c|c|c|}
\hline $\begin{array}{l}\text { SFLOUL } \\
440.00^{2}\end{array}$ & 35.11 & 34.36 & 0.00 & $6 \% 5.75$ & -0.020 & $-1: 4.7$ & $-0.04 \%$ & -0.03 & $-0.00 \%$ & 1001.5001 & 10101.16110 & \multicolumn{2}{|c|}{ 44. $31 \%$} \\
\hline $440.00 \mathrm{~A}$ & 34.96 & -0.357 & -10.374 & 1001.000 & 0.000 & -1.372 & 92.720 & -0.340 & $-1,7 \times 1$ & 11.100 & $00 \%$ & 0.600 & D. Who A w WTh \\
\hline $\begin{array}{l}\text { SPQOL } \\
450.0 O E\end{array}$ & 35.11 & $34 . \%$ & 0.00 & 695.75 & -0.024 & -99.5 & -0.041 & $-0.08 c$ & -0.005 & 1001.1001 & 11019.100 & \multicolumn{2}{|c|}{$4.7 .300 \mathrm{E}$} \\
\hline $450.100 \mathrm{~A}$ & 34.96 & -0.357 & -0.374 & $1001.00 \%$ & 0.000 & -1.832 & 802.727 & -10.317 & $-1.41=$ & 0.01 & init & 0.000 & 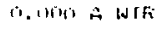 \\
\hline $\begin{array}{l}\text { SPUOL } 22 \\
460 . \text { CIOE }\end{array}$ & 35.11 & 34.6 .7 & 0.010 & $6 \% 5.75$ & -0.024 & $-108 . \therefore$ & -0.049 & -0.030 & -0.005 & 1001.1001 & 10011.100 & \multicolumn{2}{|c|}{ 44. $300 \mathrm{OF}$} \\
\hline $460.100 A$ & 34,67 & -0.357 & -0.374 & 1001 . & 0.000 & -1.892 & 32,327 & $-0 . \therefore 11$ & -1.695 & 0.00 & & 19.0100 & 11.0IT) A WTE \\
\hline $\begin{array}{l}\text { SFlUL } \\
470.00 E\end{array}$ & 34,96 & $34.9 \%$ & 0.00 & 695.75 & -0.514 & -100.6 & -0.047 & -0.038 & -0.065 & 10011.1001 & 1301.100 & \multicolumn{2}{|c|}{$44.301 \mathrm{~F}$} \\
\hline $470.00 \mathrm{~A}$ & 34,26 & -0.357 & -13.374 & 1001.009 & 0.000 & -1.832 & 092.720 & $-0,731$ & -1.1983 & $9 . m$ & & & 0.1300 \\
\hline
\end{tabular}


ENGINEERING LNITS CLNVERSION RESULTS

DATE D4-JUL-79 RUN NO. 15

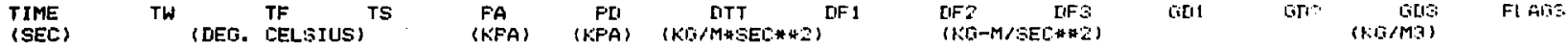

SPQOL 3

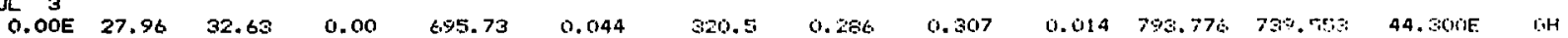

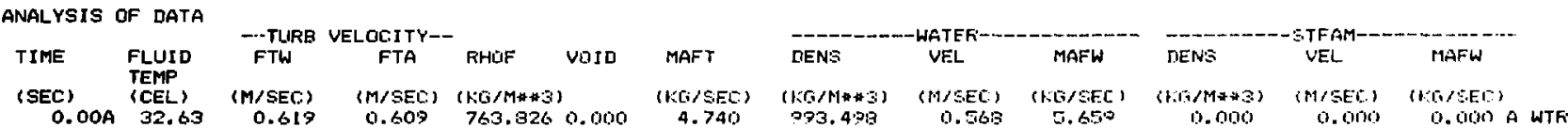

SPOOL 3

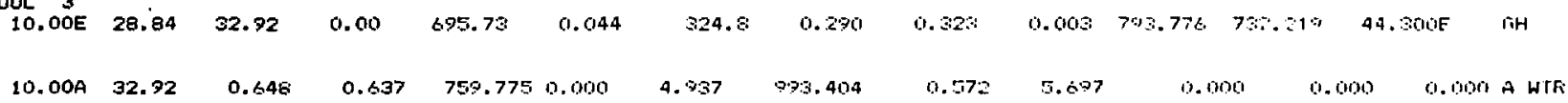

$\vec{Q}$

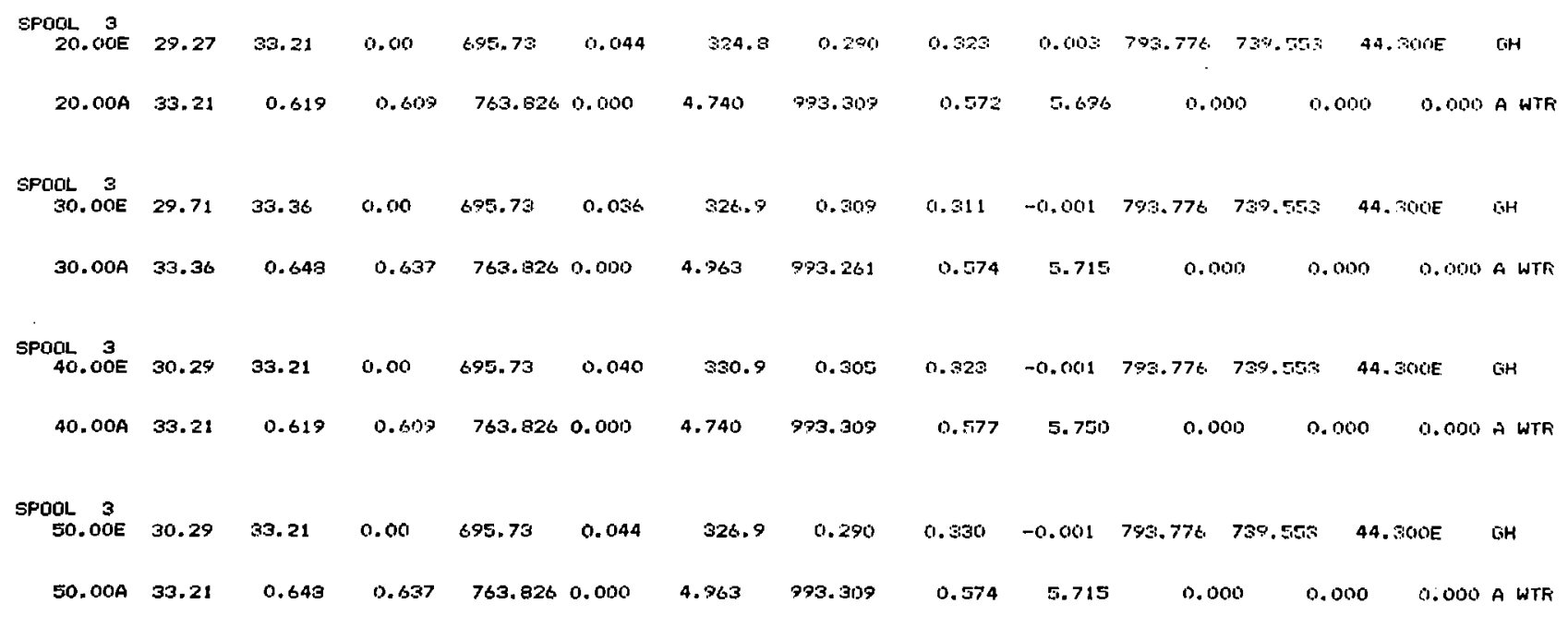




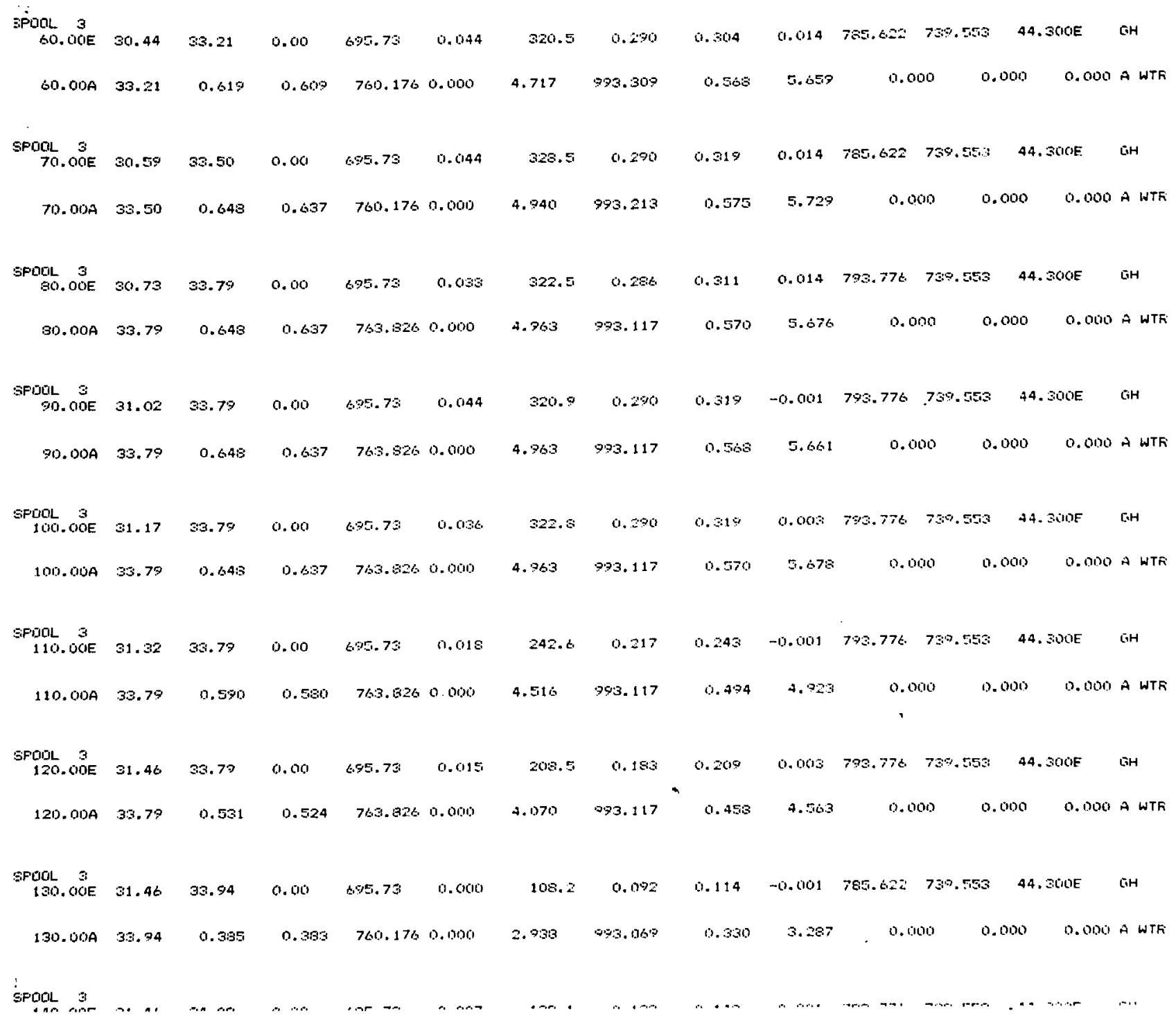




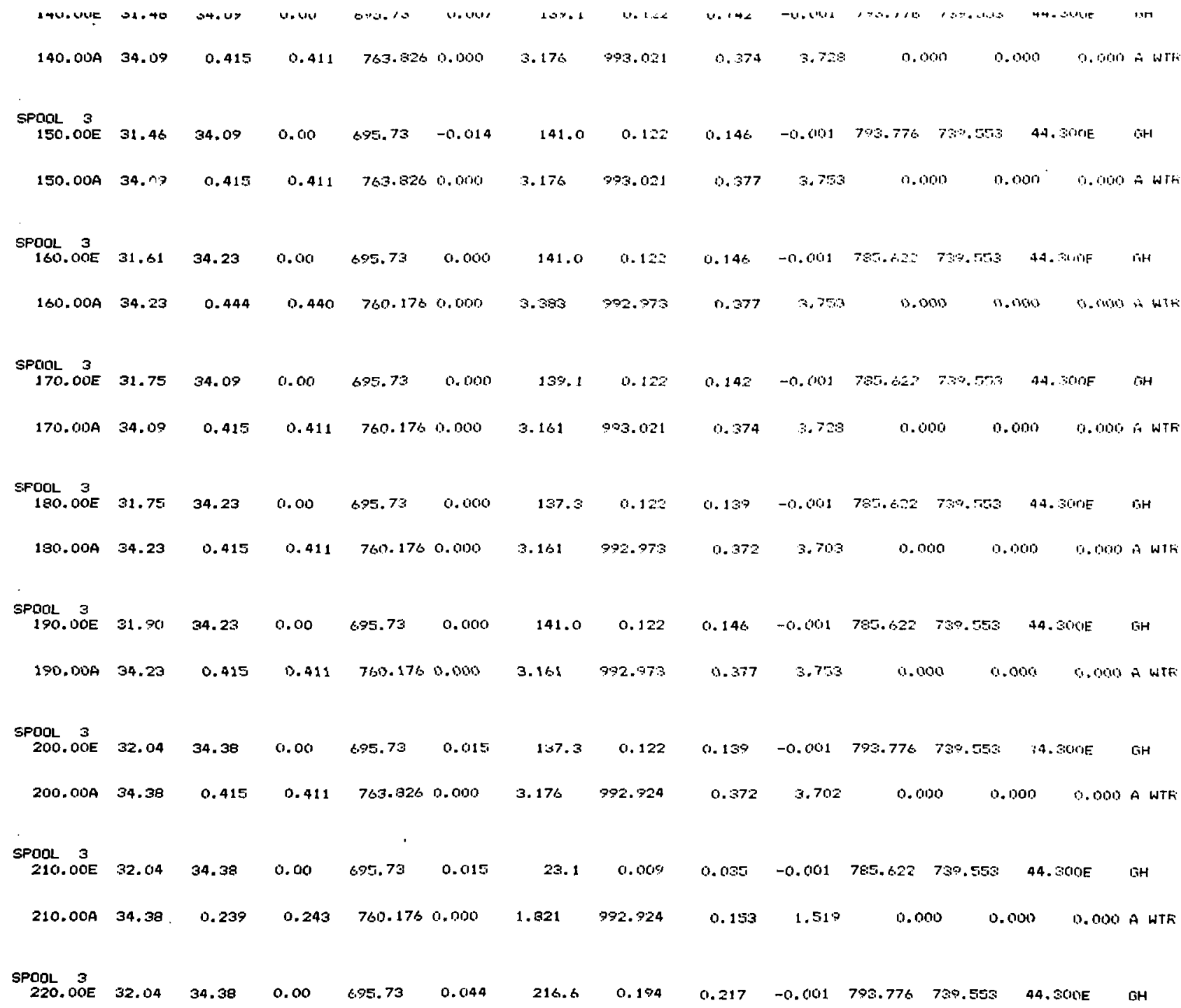




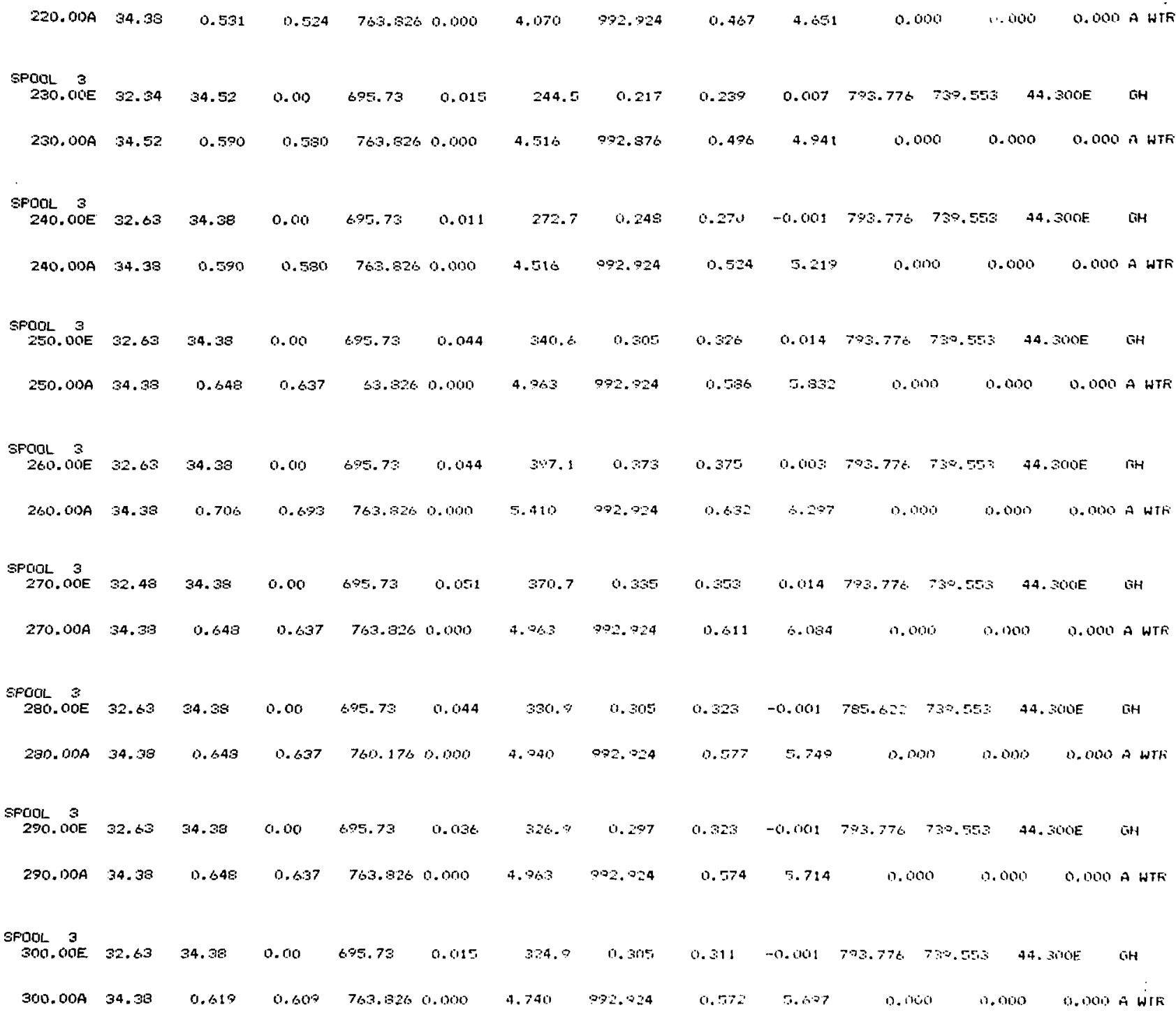




\begin{tabular}{|c|c|c|c|c|c|c|c|c|c|c|c|c|c|c|}
\hline 310. OOE & 32.77 & 34.52 & 0.00 & 695.73 & 0.044 & 328.1 & 0.250 & 0.330 & 0.001 & 703.776 & 73.553 & \multicolumn{2}{|c|}{44.30010} & $6 \mathrm{H}$ \\
\hline $310.00 \mathrm{~A}$ & 34.52 & 0.643 & 0.637 & 763.826 & 0.000 & 4.963 & 992.876 & 0.575 & 5.750 & \multicolumn{2}{|c|}{$0.000 \%$} & 0.000 & 1.9.4n! & A WTFt \\
\hline $\begin{array}{l}\text { SPQIOL } 3 \\
320.00 E\end{array}$ & 32.63 & 34.52 & 0.00 & 695.73 & 0.044 & 324.9 & $0.2 \% 7$ & 0.312 & -0.001 & 703.776 & 750,563 & & GiE & lith \\
\hline $320.00 \mathrm{~A}$ & 34.52 & 0.617 & 0.609 & 763.926 & 0.000 & 4.740 & 072.876 & 0.572 & 5.306 & \multicolumn{2}{|c|}{$0.0 \mathrm{hi}$} & לס & 0.000 & A WIE \\
\hline $\begin{array}{r}\text { SPOOL } \\
330.00 E\end{array}$ & 32.77 & 34.52 & 0.00 & 6.75 .73 & 0.044 & 324.7 & 0.293 & 0.323 & $-1 \%$. (1) & 793.776 & $74 \times, 55$ & \multicolumn{2}{|c|}{ 44. SGlite } & $\mathrm{IH}$ \\
\hline $330.00 \mathrm{~A}$ & 34.52 & 0.643 & 0.637 & 763.626 & 0.000 & 4.763 & 992.976 & 0.572 & $\because . \dot{0}=\dot{b}$ & \multicolumn{2}{|c|}{0.1300} & (in) & 0.000 & $\therefore$ WTF \\
\hline $\begin{array}{l}\text { SPOOL } 3 \\
340.0 O E\end{array}$ & 32.92 & 34.52 & 0.00 & 675.73 & 0.036. & 316.8 & 0.283 & 0.323 & -0.016 & 793.776 & 730,553 & \multicolumn{2}{|c|}{ 4a. जRlite } & $\mathrm{EHH}$ \\
\hline $340.00 \mathrm{~A}$ & 34.52 & 0.619 & 0.608 & 763.826 & 0.000 & 4.740 & 792.676 & 0.565 & $5.6 \times 5$ & \multicolumn{2}{|c|}{0.000} & (\%) & 0.60 .04 & A WTF \\
\hline $\begin{array}{l}\text { SPOOL } \\
350.00 E\end{array}$ & 32.92 & 34.67 & 0.00 & 695.73 & 0.051 & 319.9 & 0.293 & 0.311 & -0.01011 & $793.77 t$ & 754.555 & \multicolumn{2}{|c|}{$44.30 \mathrm{GIE}$} & $\mathrm{GSH}$ \\
\hline $350.00 \mathrm{~A}$ & 34.67 & 0.617 & 0.609 & 763.326 & 0.000 & 4.740 & 972.827 & 0.5 is 7 & $5 . \sin 3$ & \multicolumn{2}{|c|}{0.000} & & 0.0000 & A WTF \\
\hline $\begin{array}{l}\text { SPOOL } 3 \\
360.00 E\end{array}$ & 33.07 & 34.67 & 0.00 & 695.73 & 0.051 & 330.8 & 0.30 & 0.315 & 0.007 & 793.776 & $79 \% .553$ & \multicolumn{2}{|c|}{$44.30 \mathrm{UE}$} & $\mathrm{GH}$ \\
\hline $360.00 \mathrm{~A}$ & 34.67 & 0.619 & 0.609 & 763.326 & 0.000 & 4.740 & 792.327 & 0.577 & 5.747 & \multicolumn{2}{|c|}{0.000} & & 0.000 & Aे WTF \\
\hline $\begin{array}{l}\text { SPOOL. } 3 \\
370.00 E\end{array}$ & 33.07 & 34.67 & 0.00 & 695.73 & 0.015 & 234.6 & 0.213 & 0.232 & -0.001 & $792.77 t$ & 747.228 & \multicolumn{2}{|c|}{44.3000} & EसH \\
\hline $370.00 \mathrm{~A}$ & 34.67 & 0.590 & 0.590 & 788.066 & 0.000 & 4.542 & 992.927 & 0.486 & 4.841 & \multicolumn{2}{|c|}{0.000} & 00 & 0.000 & A WTR \\
\hline $\begin{array}{l}\text { SPOOL } \\
380.00 \mathrm{E}\end{array}$ & 32.92 & 34.96 & 0.00 & 695.73 & 0.014 & 212.6 & 0.194 & 0.209 & -0.001 & 773.778. & 739.553 & \multicolumn{2}{|c|}{ A4.300E } & GH \\
\hline
\end{tabular}




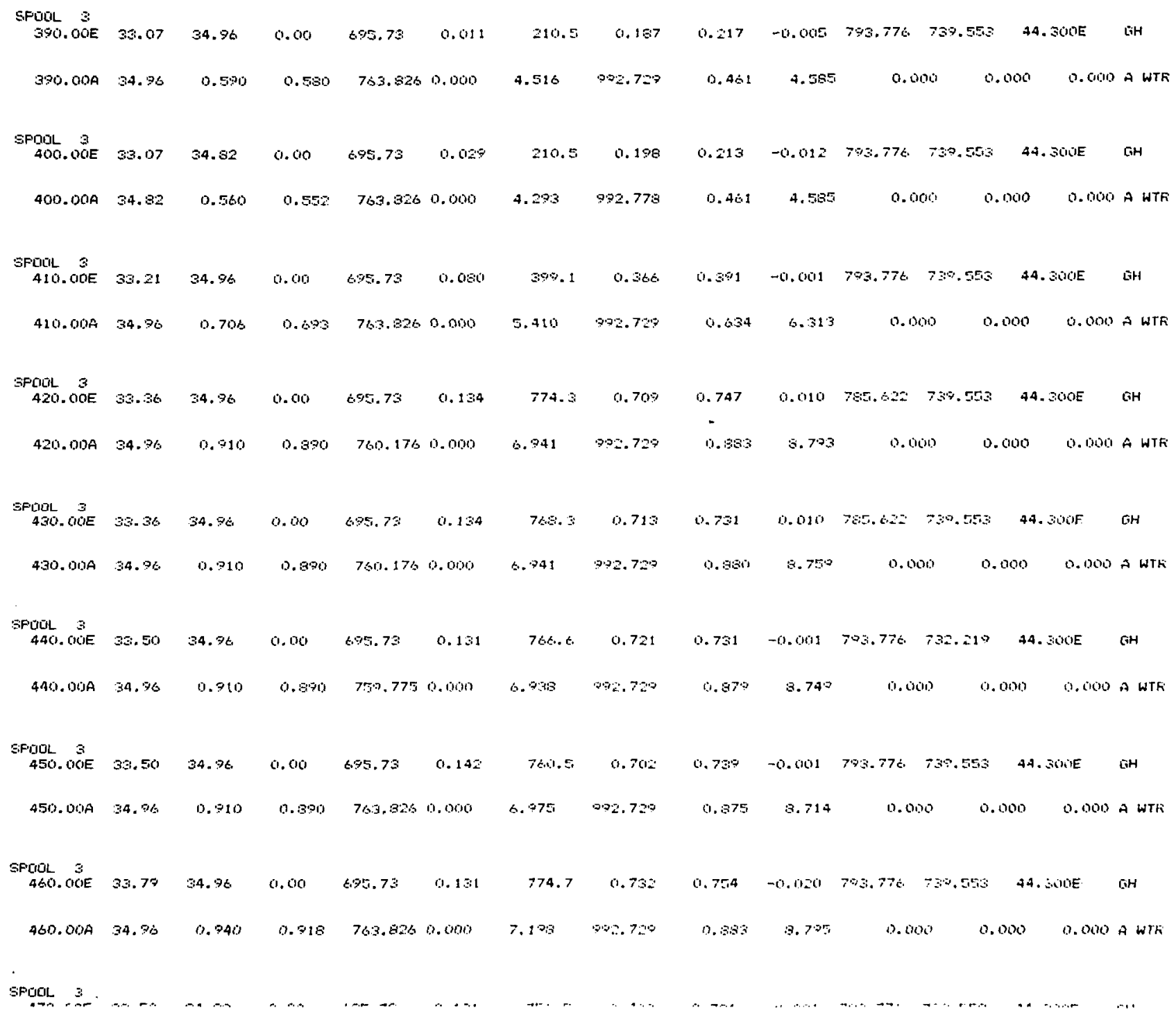




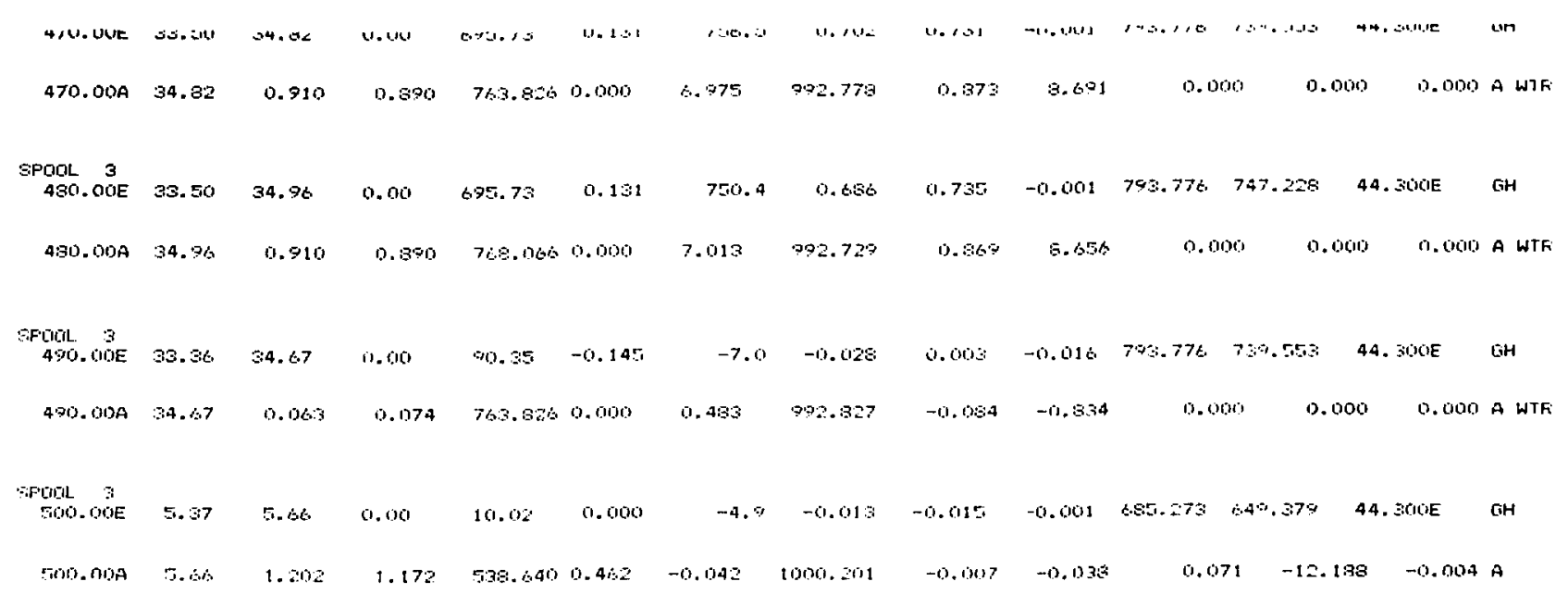

